$$
\text { IVAN DE CAMARGO E OLIVEIRA }
$$

\title{
VARIEDADES INVARIANTES PARA PONTOS FIXOS HIPERBÓLICOS
}

Tese apresentada ao Instituto de Matemática e Estatistica da U. S. P. para a obtenção do grau de Mestre 
A Ismênia 


\section{$P R E F A C O$}

O presente trabalho tem por finalidade apresentar de for ma acessível a demonstração dos Teoremas das Variedades Estável e Instável para um ponto fixo hiperbólico de um difeomorfismo $f: E \rightarrow E$ ( $E$ espaço de Banach real ou complexo ) e a correspondente versão para fluxos. A referência básica é [6], onde os autores tratam o caso mais geral em que $f$ é uma "aproximacão " na nor ma de Lipschitz - de um operador hiperbólico, e também o caso de conjuntoshiperbólicos, conceito que generaliza o de ponto fixo hiperbólico. Assim, a linguagem e as técnicas aqui empregadas preparam o terreno para essa generalização.

A teoria das veriedades estáveis e instáveis no caso de dimensão finita pode ser encontrada em [1] (apêndice ), [3] (ca pítulo XIII) ou em [5] ( capítulo IX). Em [6], encontramos a generalização para espaços de Banach: o conceito de variedades estáveis e instáveis para êsse caso é o seguinte: dada $f: E(r) \rightarrow E$ ( $E$ espaço de Banach, $E(r)$ = bola aberta com centro na origem e ra io $r$ ), "variedade estável para $f$ é o conjunto

$$
\begin{aligned}
& W^{S}=\left\{x \in E(r): f^{n}(x) \text { está definido e } f^{n}(x) \in E(x),\right. \\
& \forall n \in N\}
\end{aligned}
$$

e "variedade instável " para $f$ é o conjunto

$$
\begin{aligned}
& W^{\mathrm{L}}:=\left\{x \in E(r): f^{-n}(x) \text { está definido e } f^{-n}(x) \in E(r),\right. \\
& \left.\forall n \in \mathbb{N}^{N}\right\}
\end{aligned}
$$

E claro que $f\left(W^{s}\right) \subset W^{s} e f\left(w^{u}\right) \subset W^{u}$. Os teoremas centrais dêste trabalho garantem que se $f$ satisfaz a certas condições, existem $W^{\mathrm{S}}$ e $W^{\mathrm{U}}$, que são variedades diferenciáveis damesma classe de diferenciabilidade que $f$. Wh é obtida ( localmente, isto é, em uma bola de raio $\tilde{r}$ eventualmente menor que $r$ ) 
como gráfico de uma conveniente função, e por iteração de $f$ obtém -se $W^{\mathrm{l}}$ em tôda a bola $E(r)$ : $\mathbb{W}^{\text {S }}$ é, a grosso modo, a variedade instável para $f^{-1}$.

A matéria foi distribuída em cinco secções; na secção I, apresentamos alguns resultados básicos sôbre funçōes lipschitzia nas e funções diferenciáveis em espaços de Banach. Üm importante teorema é o de número 1.8 , que desempenhará um papel fundamental na secção 4 . Os teoremas 1.10 e 1.12 são clássicos e também terão grande utilidade na demonstração de alguns resultados importantes das seç̧ões seguintes.

A existência da secção 2 se deve ao seguinte fato: na demonstração das proposições 4.4 e 4.7, não conseguimos prescin dir da hipótese de que $f$ fôsse uniformemente $c^{k}$, ao invés de ser simplesmente de classe $C^{k}(k=1$ em 4.4$)$. Ocorre, porém, que Hirsch e Pugh não fazem em [6] essa hipótese, o que nos deixou relutantes em adotá-la, até que nos veio às mãos uma pré-impressão de [1.1], onde se demonstra o Teorema da Variedade Estável para Lipschitz - aproximações de operadores semi-hiperbólicos e onde se Iê textualmente:

"para nossa demonstração, tivemos que supor não só que a função dada fôsse diferenciável, mas também que sua derivada fôsse ( Iocal mente) uniformemente contínua. Como uma versão detalhada do artigo de Hirsch e Pugh não apareceu ainda, não tenho certeza se essas técnicas exigem a hipótese de uniformidade ".

Isso nos animou a supor $f$ uniformemente $\mathrm{c}^{k}$, hipótese essa que localmente é automática em dimensão finita se $f$ é de clas se $C^{k}$. Foi então necessário incluir a secção 2 , onde apresentamos alguns fatos a respeito de funções uniformemente $c^{k}$, utilizados na secção 4. Escrevemos a C.C.Pugh a respeito, e estamos aguardando resposta. 
A. secção 3 contém os primeiros informes a respeito de o peradores hiperbólicos e Lipschitz - perturbações, além de apresen tar a transformação-gráfico $\Gamma_{f}$, que desempenha papel vital na sec ção 4. Na proposição 3.3 aparecem os candidatos $-W_{1}$ e $W_{2}-a$ variedades instável e estável para $f$.

A seç̧ão 4 encerra os principais teoremas. Por razões pu ramente estéticas, decompusemos o Teorema da Variedade Instável em diversos lemas e proposições, que reụnidos nos dão o Teorema no ca so em que $f(0)=0 \quad(4.1,4.2,4.4,4.7$ e 4.9$)$. O Teorema 4.10 trata do caso geral, que é obtido do anterior por via formal. o corolário 4.13 se refere à situação mais importante em que $f$ é um difeomorfismo tendo um ponto fixo hiperbólico. O Teorema da Variedade Estável ( 4.14 ) é obtido aplicando o Teorema da Varieda de Instável a uma conveniente restrição de $f^{-1}$.

Finalmente, na secção 5 apresentamos alguns comentários e alternativas ao que foi feito nas seções anteriores, além de uma ou outra perspectiva de aplicação dos resultados apresentados.

Durante os meses de janeiro e fevereiro p.p., estivemos estagiando como bolsista no Instituto de Matemática Pura e Aplica da do Conselho Nacional de Pesquisas, na Guanabara, onde o contacto com os Professôres Jacob Palis Jr. e Jorge Sotomayor nos foi de inestimável valia, pelo que lhes somos profundamente agradecidos. Também foram de grande utilidade as conversas que tivemos com o Professor Sheldon E. Newhouse, do Universidade de Berkeley, E.U.A., que atualmente se encontra no I.M.P.A.

Deixamos aqui um especial agradecimento ao nosso orienta dor, Professor Waldyr Muniz Oliva, cujo incentivo nos foi de vital importância em nosso trabalho. 
Finalmente, uma palavra de reconhecimento aos professôres e colegas do Instituto de Matemática e Estatística da Universi dade de São Paulo, que de uma formạ ou de outra vêm tendo participação em nossa formação matemática.

São Paulo, abril de 1971.

Na elaboração deste trabalho contamos com o auxílio financeiro da Fundação de Amparo à Pesquisa do Estado de São Paulo. 
1. FUNÇOES IIPSCHI TZI ANAS

I.I DEFINICXO: Sejam $X$ e $Y$ dois espaços métricos. Uma função $f: X \longrightarrow Y$ é lipschitriana se existe um número real $\alpha$ tal que

$$
d(f(x), f(y)) \leqslant \alpha \cdot d(x, y), \forall x, y \in x
$$

Definimos a constante de Lipschitz de $f$ como sendo o número

$$
\begin{aligned}
& I(f)=\inf \{\alpha \in R: \quad d(f(x), f(y)) \leqslant \alpha \cdot d(x, y), \\
& \forall x, y \in X\}
\end{aligned}
$$

ou, equivalentemente,

$I(f)=\sup \left\{\frac{d(f(x), f(y))}{d(x, y)}: x, y \in X \quad x \quad x \neq y\right\}$

Se $I(f)<I$, dizemos que $f$ é uma contração.

1.2 DEFINIÇAO: Seja $X$ um espaço métrico e $f: X \rightarrow X$. Um ponts $a \in X$ é um atrator para $f$ se $f(a)=a$ e

$$
\lim _{n \rightarrow \infty} f^{n}(x)=a, \forall x \in X \text {. }
$$

É claro que nesse caso a é o único ponto fixo de $f$.

1.3 PROPOSIÇAO: Seja X um espaço métrico completo e $f: X \rightarrow X$ uma contração. Então:

(a) existe um atrator a para $f$ 
(b) se $g: X \rightarrow X$ tem um ponto fixo $b$ e se $d(f(b), g(b))<\epsilon$ então $d(a, b)$ $\frac{\epsilon}{I-I(f)}$.

Demonstração: é imediata.
Para a parte (a), ver [8], página 78. A parte (b) página 78. A parte 
Se A é um subconjunto de $Y$ contendo a origem, $\mathbb{M}(X(r), A$ ) indica o sub-espaço métrico de $\mathbb{M}(X(r), Y)$ constituído pelas funções que tomam valores em $A$; assim,

$$
\begin{aligned}
\mathbb{M}(X(r), A)=\{ & f: X(r) \rightarrow A \mid f \text { é lipschitziana e } \\
f(0)=0\} \text { e d (f,g) } & =\sup \|f(x)-g(x)\|, \\
\forall f, g \in \mathbb{M}(X(r), A) \quad & X \in X(r)
\end{aligned}
$$

Nos espaços funcionais, "d" sempre indicará uma distância definida como acima.

tão

1.5 PROPOSIÇAO: Se $\mathrm{E}_{1} \quad \theta \quad \mathrm{E}_{2}$ são espaços de Banach, en

$$
G=\left\{g \in \mathbb{M}\left(E_{1}(r), \overline{E_{2}(r)}\right): I(g) \leqslant I\right\}
$$

é um espaço métrico completc (onde $\overline{\mathrm{E}_{2}(r)}$ é a bola fechada com centro na origem e raio $r$ ).

Demonstração: a cargo do leitor.

1.6 PROPOSIÇAO: Supondo que tôdas as expressões abaixo tenham sentido, valem as seguintes propriedades:

(a) $I(f \circ g) \leqslant I(f) \cdot I(g)$

(b) $d\left(f_{1} \circ g_{I}, f_{2} \circ g_{2}\right) \leqslant d\left(f_{1}, f_{2}\right)+I\left(f_{2}\right) \cdot d\left(g_{1}, g_{2}\right)$

(c) $d\left(g^{-1}, h^{-1}\right) \leqslant I\left(g^{-1}\right) \cdot d(g, h)$

Demonstração:

A parte (a) é imediata.

Quanto a (b), temos: 
$d\left(f_{1} \circ g_{1}, f_{2} \circ g_{2}\right) \leqslant d\left(f_{1} \circ g_{1}, f_{2} \circ g_{1}\right)+d\left(f_{2} \circ g_{1}, f_{2} \circ g_{2}\right)$

$$
\leqslant d\left(f_{1}, f_{2}\right)+I\left(f_{2}\right) \cdot d\left(g_{1}, g_{2}\right) .
$$

Finalmente, aplicando (b) demonstramos (c):

$$
\begin{aligned}
d\left(g^{-1}, h^{-1}\right)= & d\left(g^{-1} \circ h \circ h^{-1}, g^{-1} \circ g \circ h^{-1}\right) \leqslant \\
\leqslant & I\left(g^{-1}\right) \cdot d\left(h \circ h^{-1}, g \circ h^{-1}\right) \leqslant \\
\leqslant & I\left(g^{-1}\right)\left[d(h, g)+I(g) \cdot d\left(h^{-1}, h^{-1}\right)\right]= \\
& I\left(g^{-1}\right) \cdot d(g, h)
\end{aligned}
$$

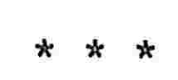

1.7 LEMA: Seja $\left(C_{n}\right)$ uma sequência de números reais não negativos tais que $C_{n} \rightarrow 0$ e seja $\xi$ tal que $0 \leqslant \xi<1$. Então

$$
\lim _{m \rightarrow \infty} \sum_{i=0}^{m} c_{i} \xi^{m-i}=0
$$

Demonstração:

Seja $m \geqslant 2$. Para todo inteiro $k$, $0<k<m$, temos:

$$
\begin{aligned}
& \left.\sum_{i=0}^{m} c_{i} \xi^{m-i}=\sum_{i=0}^{k} c_{i} \xi^{m-i}+\sum_{i=k+1}^{m} c_{i}\right\}^{m-i} \leqslant \\
& {\left[\begin{array}{l}
\max _{i} c_{i} \\
0 \leqslant i \leqslant k
\end{array}\right] \sum_{i=0}^{k} z^{m-i}+\left[\begin{array}{l}
\max c_{i} \\
k<i \leqslant m
\end{array}\right] \sum_{i=k+1}^{m} \xi^{m-i} \leqslant}
\end{aligned}
$$


9

$$
\left[\begin{array}{l}
\max c_{i} \\
0 \leqslant i \leqslant k
\end{array}\right] \frac{\xi^{m-k}}{I-\xi}+\left[\begin{array}{l}
\max _{i} \\
k<i \leqslant m
\end{array}\right] \frac{I}{I-\xi}
$$

Seja $\mathbb{M} \in R$ tal que $C_{i} \leqslant \mathbb{M}, \forall i$

Então:

$$
\sum_{i=0}^{m} a_{i} \xi^{m-i} \leqslant \frac{M}{1-\xi} \cdot \xi^{m-k}+\frac{1}{1-\xi} \cdot\left[\begin{array}{l}
\max c_{i} \\
k<i \leqslant m
\end{array}\right]
$$

Tomando $k=\left[\frac{m}{2}\right]$, temos:

$$
m \rightarrow \infty \Longleftrightarrow k \rightarrow \infty \Longleftrightarrow m-k \rightarrow \infty
$$

e portanto

$$
\begin{aligned}
& 0 \leqslant \lim _{m \rightarrow \infty} \sum_{i=0}^{m} c_{i} \xi^{m-i} \leqslant \lim _{m \rightarrow k \rightarrow \infty} \frac{M}{1-\xi} \xi^{m-k}+ \\
& +\lim _{k \rightarrow \infty} \frac{2}{1-\xi} \cdot\left[\begin{array}{cc}
\max & c_{i} \\
k<i \leqslant m
\end{array}\right]=0
\end{aligned}
$$

1.8 TEOREMA ( da contração em fibras )

Sejam $X$ e $Y$ dois espaços métricos e $f: X \rightarrow X$ com 
um atrator p. Seja $\left(g_{X}\right)_{X} \in X$ uma família de funções lipschit zianas $g_{X}: Y \rightarrow Y$ tais que

(I.I) $\lim _{n \rightarrow \infty} \sup I\left(g_{f^{n}(x)}\right)<I, \forall x \in X$

e, para cada $y \in Y$, a função

$$
\begin{aligned}
u: X & \rightarrow Y \\
X & \mapsto g_{X}(y)
\end{aligned}
$$

seja contínua. Se $g_{p}$ tem um ponto fixo $q$, então $(p, q)$ é um atrator para a função

$$
\mathrm{F}: \mathrm{X} \times \mathrm{Y} \longrightarrow \mathrm{X} \times \mathrm{Y}
$$

definida por $F(x, y)=\left(f(x), g_{x}(y)\right)$

Demonstração:

A condição ( $I, I$ ) acarreta que, para cada $x \in X$, existe $r \in \mathbb{N}, \quad r=r(x)$, tal que

$$
n \geqslant r \Rightarrow I\left(g_{f}^{n}(x) \leqslant \lambda<1\right.
$$

onde

$$
\lambda=\sup _{n \geqslant r} I\left(g f^{n}(x)\right.
$$

Como $\mathrm{p}$ é atrator de $\mathrm{f}$, basta demonstrar que 
$\lim d\left(\pi_{2} \circ F^{n+I}(x, y), q\right)=0, \forall(x, y) \in X \times Y$ $n \rightarrow \infty$

onde $\pi_{2}: X \times Y \rightarrow Y$ é a projeção no segundo fator. Notemos ainda que

$$
F^{n+1}(x, y)=\left(f^{n+1}(x), g_{f^{n}}(x) g_{f^{n-1}(x)}^{\circ} \cdots g_{f(x)}^{\circ} g_{x}(y)\right)
$$

Fixado um ponto $(x, y) \in X \times Y$, ponhamos, para cada $n \in \mathbb{N}$, $n \geqslant r(x)$

$$
\delta_{n}=d\left(g_{f} n_{(x)}(q), q\right) .
$$

Da hipótese de continuidade da função u, concluímos que lim $\delta_{n}=0$. Então, para $n>r(x)$, temos: $n \rightarrow \infty$

(I.II $\quad d\left(\pi_{2} \circ F^{n+I}(x, y), q\right) \leqslant$

$$
\begin{aligned}
& \leqslant d\left(\pi_{2} \circ F^{n+1}(x, y), \pi_{2} \circ F^{n+1}(x, q)\right)+ \\
& +d\left(\pi_{2} \circ F^{n+1}(x, q), q\right) .
\end{aligned}
$$

Analisemos cada uma das parcelas do segundo membro:

$$
a\left(\pi_{2} \circ F^{n+1}(x, y), \pi_{2} \circ F^{n+1}(x, q)\right) \leqslant
$$


12

$$
\begin{aligned}
& I\left(g_{f}(x)\right) \cdot d\left(\pi_{2} \circ F^{n}(x, y), \pi_{2} \circ F^{n}(x, q)\right) \leqslant \\
& \cdots \leqslant \prod_{i=r}^{n} \quad I\left(g_{f^{i}(x)}\right), \alpha(x, y)
\end{aligned}
$$

onde $\alpha(x, y)=a\left(\pi_{2} \circ F^{r}(x, y), \pi_{2} \circ F^{r}(x, q)\right)$. Logo,

$$
d\left(\pi_{2} \cdot \mathrm{F}^{n+1}(x, y), \pi_{2} \circ \mathrm{F}^{n+1}(x, q)\right) \leqslant \lambda^{n-r+1} \cdot \alpha(x, y) .
$$

Quanto à segunda parcela,

$$
\begin{aligned}
& d\left(\pi_{2} \circ F^{n+1}(x, q), q\right) \leqslant \\
& \leqslant d\left(\pi_{2} \circ F^{n+1}(x, q), g_{f^{n}(x)}(q)\right)+ \\
& +d\left(g_{f^{n}(x)}(q), q\right) \leqslant \\
& I\left(g_{f} n_{(x)}\right) \cdot d\left(\pi_{2} 0 F^{n}(x, q), q\right)+\delta_{n} \leqslant \\
& \lambda\left[a\left(\pi_{2} \circ F^{n}(x, q), g_{f^{n-1}(x)}(q)\right)+\delta_{n-1}\right]+\delta_{n} \leqslant \\
& \leqslant \cdots \leqslant \lambda^{n-r+1} \cdot \beta(x)+\sum_{j=r}^{n} \lambda^{n-j} S_{j}
\end{aligned}
$$


onde $\beta(x)=d\left(\pi_{2} 0 F^{r}(x, q), q\right)$.

De (I.II) podemos então concluir:

(I.III) $d\left(\pi 2^{\circ} \mathrm{F}^{\mathrm{n}+I}(\mathrm{x}, \mathrm{y}), \mathrm{q}\right) \leqslant$

$$
\leqslant[\alpha(x, y)+\beta(x)] \lambda^{n-r+1}+\sum_{j=r}^{n} \lambda^{n-j} \delta_{j}
$$

Fazendo, no lema $1.7, \xi=\lambda, \quad C_{n}=S_{n+r}, \quad i=j-r \quad$ e $m=n-r$, vê-se claramente que o segundo membro de (I.III) tende a zero.

1.9 LEMA: Seja E um espaço normado; U $U \mathrm{E}$ aberto $e$ $f \quad: U \rightarrow E$ uma função lipschitziana e diferenciável. Então, pa ra todo $x \in U,\|D f(x)\| \leqslant I(f)$ e conseqüentemente, se $U$ é convexo, temos pelo Teorema do Valor Médio que

$$
L(f)=\sup _{x \in U}\|D f(x)\|
$$

Demonstração:

E fácil ver que para todo $x \in U$ e $h \in E$, temos:

$$
D f(x) h=\lim _{t \rightarrow 0} \frac{f(x+t h)-f(x)}{t}
$$




$$
\begin{aligned}
& \|D f(x)\|=\sup \|D f(x) \cdot h\|= \\
& \|h\| \leqslant 1 \\
& =\sup _{\|h\| \leqslant I} \lim _{t \rightarrow 0} \frac{\|f(x+t h)-f(x)\|}{|t|} \\
& \sup _{\|h\| \leqslant I} \lim _{t \rightarrow 0} \frac{I(f) \cdot|t| \cdot\|h\|}{|t|}= \\
& =\sup I(f) \cdot\|h\|=I(f) \\
& \|h\| \leqslant 1
\end{aligned}
$$

1.10 TEOREMA ( da perturbação de um isomorfismo).

Seja $E$ um espaço de Banach e $f: U C E \rightarrow E$, U aber to. Seja $\mathrm{T}: \mathrm{E} \rightarrow \mathrm{E}$ um isomorfismo contínuo tal que $f-T$ seja lipschitziana, com $I(f-T)<\left\|T^{-1}\right\|^{-I}$. Então:

(a) $f$ é um homeomorfismo de $U$ sôbre um aberto de $\mathbb{E}$.

(b) $I\left(f^{-1}\right) \leqslant\left[\left\|T^{-1}\right\|-1-I( \pm-T)\right]^{-1}$

(c) Se f fôr diferenciável, então $f$ é um difeomorfismo.

Demonstração:

(a) Em [8] , página 80, é feita uma demonstração que se adapta perfeitamente para espaços de Banach em geral.

(b) $\forall x, y \in U$, temos: 


$$
\|f(x)-f(y)\|=\|T x-T y+(f-T)(x)-(f-T)(y)\| \geqslant
$$

$$
\begin{aligned}
& \|T x-T y\|-\|(f-T)(x)-(f-T)(y)\| \geqslant \\
& {\left[\left\|T^{-1}\right\|^{-1}-I(f-T)\right]\|x-y\|}
\end{aligned}
$$

Pondo $\quad f(x)=a \quad e \quad f(y)=b$, segue-se que, $\forall a, b \quad f f(U)$

$$
\left\|f^{-1}(a)-f^{-1}(b)\right\| \leqslant\left[\left\|T^{-I}\right\|^{-1}-I(f-T)\right]^{-1}\|a-b\|
$$

donde $\quad I\left(f^{-1}\right) \leqslant\left[\left\|T^{-1}\right\|^{-1}-L(f-T)\right]^{-1}$

(c) Para todo $x \in U$, temos:

$$
D(f-T)(x)=D f(x)-T
$$

Então, pelo lema anterior, segue-se que

$$
\begin{aligned}
& L(D f(x)-T)=\|D f(x)-T\|=\|D(f-T)(x)\| \leqslant \\
& \leqslant I(f-T)<\left\|T^{-1}\right\|^{-1}
\end{aligned}
$$

e portanto, pela parte (a), D $f(x)$ é um isomorfismo, o que prova que $f$ é difeomorfismo (Teorema da Função Inversa).

A demonstração da parte (a) do teorema anterior nos fornece um dado importante, que enunciamos na seguinte 
1.11 PROPOSIÇAO Nas condições do teorema 1.10, se a bola fechada $\bar{A}$ de centro $a \in E$ e raio $r$ estiver contida em $U$, então a bola aberta de centro $b=f(a)$ e raio $\left[\left\|T^{-1}\right\|^{-1}-I(f-T)\right] r \quad$ está contida em $f(U)$.

1.12 TEORIMA. Seja A um aberto de um espaço de Banach $E$ e $\left(f_{n}\right)$ uma sequência de funções diferenciaveis definidas em A, com valores num espaço de Banach $F$. Suponhamos que: (i) existe um ponto $a \in A$ tal que a sequência $\left(f_{n}(a)\right)$ converge em $F$; (ii) Para todo ponto $x \in A$, existe uma bola $B(x)$ de centro $x$, contida em $A$, tal que a sequência $\left(D f_{n}\right)$ converge uniformemen te em $B(x)$.

Então, para cada $x \in A$, a sequência $\left(f_{n}\right)$ oonverge uniformemente em $B(x)$; além disso, se para cada $y \in A$ pusermos

$$
f(y)=\lim _{n \rightarrow \infty} f_{n}(y) e \quad g(y)=\lim _{n \rightarrow \infty} D f_{n}(y), \quad \text { então }
$$

$g(y)=D f(y), \quad \forall y \in A_{0}$

Demonstração: ver [4], capítulo VIII, $\S 6$.

1.13 LEMA. Seja E um espaço de Banach, $V$ uma vizi nhança da origem e $f: V \rightarrow E$ uma função de classe $C^{l}$ tal que $f(0)=0$ e $\quad D f(0)=0$. Para todo $\epsilon>0$, existe uma vizinhan ça $U \subset V$ da origem tal que $f / U$ tem constante de Iipschitz me nor que $\varepsilon$.

Demonstração: ver [9], Lema 2. 
2. FUNÇOES UNI FORMENEINTE $\mathrm{C}^{\mathrm{K}}$

Obs:- No que segue, sendo $E$ e $F$ espaços normados, $E \times F$ sem pre indicará o espaço-produto com a norma.

$$
\|(x, y)\|=\max \{\|x\|,\|y\|\}
$$

2.I DEFINIÇAO. Sejam $\mathrm{E}$ e $\mathrm{F}$ espaços normados $\theta$ U CE um aberto. Uma função $f: U \rightarrow F$ é uniformemente $C^{k}$ (escreve mos $\left.f \in U G^{k}\right)$ se, e sòmente se, $f \in C^{k} \quad e$ $f, D f, D^{2} f, \ldots, D^{k_{f}}$ são uniformemente contínuas.

2.2 PROPOSIÇAO. Sejam $E$ e $F$ dois espaços normados e $U C$ limitado e convexo, Se $h: U \rightarrow F$ é uniformemente continua, então h é limitada.

Demonstração:

seja $\delta>0$ tal que

Seja $D=$ diam $U$ e fixemos um ponto a $\in U$. Dado $\varepsilon_{-}>0$,

$$
\|u-v\|<\delta \Longrightarrow\|h(u)-h(v)\|<\varepsilon
$$

Podemos então, para todo $x \in U$, tomar sôbre o segmento $[x, a] C U$ um número finito de pontos $x_{0}=x, x_{1}, \ldots, x_{n}=a$, não superior a $Q=\left[\frac{D}{S}\right]+1$, tais que

$$
\left\|x_{i}-x_{i-1}\right\|<\delta \quad(i=1,2, \ldots, n)
$$

donde $\left\|h\left(x_{i}\right)-h\left(x_{i-1}\right)\right\|<\varepsilon \quad(i=1,2, \ldots, n)$ 
Então:

$$
\begin{aligned}
& \|h(x)-h(a)\|=\left\|\sum_{i=1}^{n}\left[h\left(x_{i}\right)-h\left(x_{i-1}\right)\right]\right\| \leqslant \\
& \leqslant \sum_{i=1}^{n}\left\|h\left(x_{i}\right)-h\left(x_{i-1}\right)\right\|<n \cdot \varepsilon \leqslant Q \cdot \varepsilon
\end{aligned}
$$

e portante

$$
\|h(x)\|<\|h(a)\|+Q E \quad, \forall x \in U
$$$$
\text { *** }
$$

2.3 COROLARIO. Se $\mathrm{I}$ e F são espaços normados e UCE é um abert convexo e limitado, então $f: U \rightarrow F$ é unifoṛmemente $C^{k}$ se, e sòmente se, $D^{k} f$ fôr uniformemente contínua. Demonstração:

Pela desigualdade do valor médio, temos, $\forall x, y \in U:$

$$
\left\|D^{k-1} f(x)-D^{k-1} f(y)\right\| \leqslant \sup _{u \in[x, y]}\left\|D^{k} f(u)\right\| \cdot\|x-y\|
$$

Supondo então $D^{k} f$ uniformemente contínua, temos pela proposição $\iota .2$ que existe $\mathbb{M}>0$ tal que $\left\|D^{k_{f}}(u)\right\| \leqslant M$ $\forall u \in U ;$ dar,

$$
\left\|D^{k-1} f(x)-D^{k-1} f(y)\right\| \leqslant \mathbb{M} \quad\|x-y\|, \forall x, y \in U
$$

e portanto $D^{k-1} f$ é uniformemente contínua. Repetindo o argumen 
to, provamos que $D^{k-2} f, \ldots, D f$ e $f$ são uniformemente contínuas.

A recíproca está implíoita na definição 2.1

normados e

2.4 PROPOSIÇAOO. Sejam E, $F_{1}, F_{2}, \ldots, F_{n}$,$H$ espaços

$$
\rho: \mathrm{F}_{1} \times \mathrm{F}_{2} \times \ldots \times \mathrm{F}_{\mathrm{n}} \longrightarrow \mathrm{H}
$$

uma aplicação n-linear contínua. Indicaremos

$$
\rho\left(a_{1}, a_{2}, \ldots, a_{n}\right)=a_{1} a_{2}, \ldots a_{n}
$$

Sejam U $\subset E$ um aberto oonvexo e limitado e

$$
\alpha_{i}: U \longrightarrow F_{i} \quad(i=I, \ldots, n)
$$

n funções uniformemente contínuas.

Definamos

$$
\alpha=\alpha_{1} \alpha_{2} \ldots \alpha_{n}: u \rightarrow H
$$

por $\alpha(x)=p\left(\alpha_{I}(x), \ldots, \alpha_{n}(x)\right)=$

$$
\alpha_{1}(x) \alpha_{2}(x) \ldots \alpha_{n}(x)
$$

Então: $\alpha$ é uma função uniformemente contínua.

Demonstração:

Se $x, y \in U$, temos: 
20

$$
\begin{aligned}
& \|\alpha(x)-\alpha(y)\|=\| \alpha_{1}(x) \alpha_{2}(x) \ldots \alpha_{n}(x)-\alpha_{1}(y) \alpha_{2}(y) \ldots \\
& \ldots \alpha_{n}(y) \| \leqslant \\
& \leqslant\left\|\alpha_{1}(x) \alpha_{2}(x) \ldots \alpha_{n}(x)-\alpha_{1}(y) \alpha_{2}(x) \ldots \alpha_{n}(x)\right\|+ \\
& +\left\|\alpha_{1}(y) \alpha_{2}(x) \ldots \alpha_{n}(x)-\alpha_{1}(y) \alpha_{2}(y) \alpha_{3}(x) \ldots \alpha_{n}(x)\right\|+ \\
& +\ldots+\left\|\alpha_{1}(y) \alpha_{2}(y) \ldots \alpha_{n-1}(y) \alpha_{n}(x)-\alpha_{1}(y) \alpha_{2}(y) \ldots \alpha_{n}(y)\right\|= \\
& =\left\|\left[\alpha_{1}(x)-\alpha_{1}(y)\right] \alpha_{2}(x) \ldots \alpha_{n}(x)\right\| \| \\
& +\left\|\alpha_{1}(y)\left[\alpha_{2}(x)-\alpha_{2}(y)\right] \alpha_{3}(x) \ldots \alpha_{n}(x)\right\|+\ldots+ \\
& +\left\|\alpha_{1}(y) \alpha_{2}(y) \ldots \alpha_{n-1}(y)\left[\alpha_{n}(x)-\alpha_{n}(y)\right]\right\| \leqslant \\
& \leqslant\|\rho\| \cdot\left\|\alpha_{1}(x)-\alpha_{1}(y)\right\| \cdot\left\|\alpha_{2}(x)\right\| \ldots\left\|\alpha_{n}(x)\right\|+ \\
& +\|\rho\| \cdot\left\|\alpha_{1}(y)\right\| \quad \cdot\left\|\alpha_{2}(x)-\alpha_{2}(y)\right\| \cdot\left\|\alpha_{3}(x)\right\| \ldots \\
& \ldots\left\|\alpha_{n}(x)\right\| \quad+\quad \ldots+\|\rho\| \cdot\left\|\alpha_{1}(y)\right\| \cdot\left\|\alpha_{2}(y)\right\| \ldots\left\|\alpha_{n}(x)-\alpha_{n}(y)\right\| \\
& +\ldots+
\end{aligned}
$$


Mas, por 2.2, existe $\mathbb{M}>0$ tal que

$\left\|\alpha_{i}(z)\right\| \leqslant \mathbb{M}, \forall z \in U(i=1, \ldots, n)$. Por outro lado as $\alpha_{i}$ sendo uniformemente continuas, para todo $\epsilon>0$, existe $\delta>0$ tal que

$$
\|x-y\|<\delta \Rightarrow\left\|\alpha_{i}(x)-\alpha_{i}(y)\right\|<\frac{e}{n\|\rho\| M^{n-1}}
$$

$(i=1, \ldots, n) \quad($ se $\rho=0, \alpha$ é a aplicação nula e a prope sição vale trivialmente).

Conclusão:

$$
\begin{aligned}
& \|x-y\|<\delta \Longrightarrow\|\alpha(x)-\alpha(y)\|<n\|\rho\| \mathbb{M}^{n-1} \cdot \frac{\epsilon}{n\|\rho\| \mathbb{M}^{n-1}}=\epsilon \\
& 2.5 \text { NOTAÇOES. Sejam } E, F \text { espaços normados }{ }^{\prime} I^{k}(E, F)
\end{aligned}
$$

- espaço normado das aplicações $k$-lineares contínuas de $\mathrm{E}$ em $\mathrm{F}$. Se $A \in L^{k}(E, F) \quad e \quad h, h_{1}, \ldots, h_{k} \in E$, indicamos por

$A h_{1} h_{2} \ldots h_{k}$ o valor de A no ponto $\left(h_{1}, h_{2}, \ldots, h_{k}\right)$ e por $A h^{k}$ o valor de A no ponto $(h, h, \ldots, h)$

Sejam agora $F_{1}, F_{2}, \ldots, F_{q} \odot G$ espacos normados,

$B \in I^{q}\left(F_{1}, \ldots, F_{q} ; G\right), A_{j} \in I^{i} \cdot j\left(E, F_{j}\right)$

$(j=1,2, \ldots ., q)$ e $k=i_{1}+\ldots+i_{q} \cdot$ A função 
$B A_{1} A_{2} \ldots, A_{q} \in L^{k}\left(E, G_{g}\right)$ é definida por

B. $A_{1} A_{2} \ldots A_{q} h_{1} h_{2} \ldots h_{k}=B\left(A_{1} h_{1} \ldots h_{i_{1}}\right)\left(A_{2} h_{i_{1}+1} \ldots h_{i_{1}+i_{2}}\right)$

$\ldots\left(A_{q} h_{i_{1}}+\ldots+i_{q-1}+1 \ldots h_{k}\right)$ para $h_{1}, h_{2}, \ldots, h_{k} \in E$.

O leitor demonstrará fàcilmente a

2.6 PROPOSIÇAO. A aplicação

$\rho: I^{q}\left(F_{I}, \ldots F_{q} ; G\right) \times I^{i_{I}}\left(E, F_{I}\right) \times \ldots \times I^{i} q\left(E, F_{q}\right) \rightarrow L^{k}(E, G)$

definida por

$$
\rho\left(B, A_{1}, A_{2}, \ldots, A_{q}\right)=B A_{1} A_{2} \ldots A_{q}
$$

é q +1 -linear e contínua.

2.7 PROPOSI CAO. Sejam E,F e G espacos normados, $U \subset E$ e $V \subset F$ abertos, $g: U \rightarrow V, f: V \rightarrow G$ funções de classe $\mathbb{C}^{r}$. Então, fog é de classe $\mathbb{C}^{r}$ e para $k=0,1, \ldots, r$ e $x \in U$, tem-se

$$
\begin{aligned}
& D^{k}(f \circ g)(x)= \\
& =\sum_{1 \leqslant q \leqslant k} \sum \delta_{k}\left(i_{1}, \ldots, i_{q}\right) \quad D q_{f}(g(x)) D^{i_{1}} l_{g}(x) \ldots D^{i_{g}} q_{g}(x)
\end{aligned}
$$

onde a segunda somatoria se estende sôbre tôdas as q - uplas de 
números inteiros positivos $\left(i_{1}, \ldots, i_{q}\right)$ tais que $i_{1}+\ldots+i_{q}=k$ e $\sigma_{k}\left(i_{1}, \ldots i_{q}\right)$ é o número inteiro defini do por recorrência do seguinte modo: $\sigma_{I}(I)=I$ e se já estiver definido $\sigma_{q}\left(i_{l}, \ldots, i_{j}\right)$ para tôdas as $j$-uplas de inteiros posi tivos $\left(i_{1}, \ldots, i_{j}\right) \operatorname{com} i_{1}+\ldots+i_{j}=q$, para $1 \leqslant q \leqslant k$, en tão

$$
\sigma_{k+1}(k+1)=1
$$

e $\sigma_{k+1}\left(i_{1}, \ldots, i_{j}, k-q+1\right)=\left(\begin{array}{c}k \\ q\end{array}\right) \Im_{q}\left(i_{1}, \ldots, i_{j}\right)$ Demonstração: ver $[2]$, página 3.

2.8 PROPOSI ÇAO. Sejam E,F, e G espaços normados, $U \subset E$ aberto, convexo e limitado, $V \subset F$ aberto e sejam $f: V \rightarrow G, g: U \rightarrow V$. Se $f$ e $g$ são funções uniformemente $C^{r}$, então fog é uniformemente $C^{r}$. Êsse resultado se generaliza triví almente para $n$ funções uniformemente $C^{r}$.

Demonstração:

Para cada $x \in U$, façamos em 2.5,

$B=D q_{f o g}(x), F_{I}=F_{2}=\ldots=F_{q}=F, A_{j}=D^{i} j_{g}(x)$. Na proposição 2.4, ponhamos $F_{1}=I q(F, G), F_{2}=I^{i} I_{(E, F)} \ldots F_{q+I}=I^{i} q_{(E, F)}$ $H=I^{k}(E, G), \quad \int$ como na proposição 2.6 e ainda $\alpha_{1}=D^{q_{\text {fog }},}, \alpha_{2}=D^{i}{ }^{1} g, \ldots, \alpha_{q+1}=D^{i} q_{g}$.

Por hipótese, para $1 \leqslant k \leqslant r$, as funções $\alpha_{i}$ são tôdas uniformemen 
te contínuas. Daí, pela proposição 2.4, temos que a função

$$
\alpha: U \longrightarrow I^{k}(E, G)
$$

tal que

$$
\alpha(x)=D^{q_{f}}(g(x)) D^{i} I_{g(x)} \ldots D^{i} q_{g}(x)
$$

é uniformemente contínua, e portanto $\sigma_{k}\left(\dot{i}_{1}, \ldots, i_{q}\right) \propto$ é tam bém uniformemente contínua. Concluímos que a função

$$
D^{k}(f \circ g): U \longrightarrow L^{k}(E, G)
$$

é uniformemente contínua para todo $k$ tál que $1 \leqslant k \leqslant r$, uma vez que é soma de funções uniformemente continnuas de $U$ em $L^{k}(E, G)$. 0 caso $r=0$ pode ser demonstrado diretamente com facilidade.

2.9 NOTAÇOES. Sejam $E$ e F dois espaços normados. Se $\mathrm{U} \subset \mathrm{E}$ é um aberto convexo, indicaremos por $\mathrm{C}^{\mathrm{k}}(\mathrm{U}, \mathrm{F})$ o espaço veto rial das funções $f: U \rightarrow F$, de classe $C^{k}$, tais que

$|g|_{k}=\sup _{u \in U} \max \left\{\|f(u)\|,\|D f(u)\|, \ldots,\left\|D^{k_{f}}(u)\right\|\right\}<\infty$

t rotina verificar que ||$_{k}$ é uma norma sôbre êsse espą ço e que se $F$ é um espaço de Banach, o mesmo se dá com $C^{k}(U, F)$.

2.10 TEOREMA . Seja

$$
\mathrm{v}: \mathrm{C}^{\mathrm{k}}(\mathrm{U}, \mathrm{F}) \times \mathrm{U} \rightarrow \mathrm{F}
$$

a "função valoração ", definida por

$$
V(f, x)=f(x)
$$


Então : $v$ é de classe $c^{k}$, e para $p=1,2, \ldots, k$, temos:

$$
\begin{aligned}
& D^{p} v(f, x)\left(g_{1}, y_{1}\right) \ldots\left(g_{p}, y_{p}\right)= \\
= & D^{p} f(x) y_{1} \ldots y_{p}+\sum_{i=1}^{p} D^{p-1} g_{i}(x) y_{1} \ldots \hat{y}_{i} \ldots y_{p}
\end{aligned}
$$

$\operatorname{com} g_{i}, f \in C^{k}(U, F) \quad e \quad x, y_{i} \in U \quad(i=1, \ldots, p)$

Demonstração: ver [2], página 25, teorema 10.3.

2.11 PROPOSIÇO. A f unção valoração

$$
\begin{array}{r}
v: I(E, F) \times E \longrightarrow F \\
(f, x) \longmapsto f(x)
\end{array}
$$

é uniformemente $\mathrm{C}^{\infty}$.

Demonstração:

Basta notar que $v$ é uma aplicação bilinear contínua, uma vez que

$$
\|v(f, x)\|=\|f(x)\| \leqslant\|f\| \cdot\|x\|
$$

2.12 PROPOSIÇÃO. Sejam $E$ e $F$ espaços normados, $U \subset \mathbb{E}$ um aberto convexo e $f: U \rightarrow F$ uma função uniformemente $C^{l}$. Para todos $a, x \in U$ tais que $x+a \in U$, seja

$$
r(x, a)=f(x+a)-f(x)-D f(x) \cdot a \text {. }
$$


Então, $\frac{r(x, a)}{\|a\|} \rightarrow 0$ com a, uniformemente em $x$, isto é,

$\forall \varnothing>0, \exists \delta>0$ tal que $\|a\|<\delta \Rightarrow \frac{\|r(x, a)\|}{\|a\|}<\varepsilon, \quad \forall x \in U$

Demonstração :

Para cada $x \in U$, consideremos a função $g_{\mathrm{X}}: U \rightarrow F$ defi nida por

$$
g_{X}(t)=f(t)-D f(x) t \quad, \forall t \in U
$$

E claro que

$$
D_{g_{X}}(t)=D f(t)-D f(x), \forall t \in U
$$

e portanto $g_{X} \in U C^{1}$. Pela desigualdade do valor médio, temos:

$$
\left\|g_{X}(x+a)-g_{x}(x)\right\| \leqslant\|a\| \sup _{t \in[x, x+a]}\left\|D g_{x}(t)\right\|
$$

ou $\|f(x+a)-D f(x)(x+a)-f(x)+D f(x) x\| \leqslant$

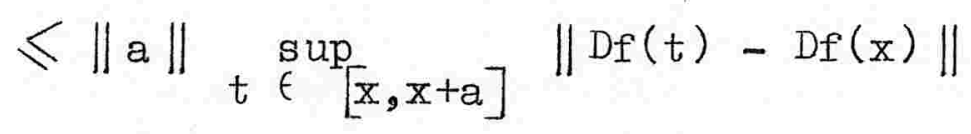

ou ainda

$$
\|f(x+a)-f(x)-D f(x) a\| \leqslant\|a\| \sup _{t \in[x, x+a]}\|D f(t)-D f(x)\|
$$

donde 


$$
\frac{\|r(x, a)\|}{\|a\|} \leqslant \sup _{t \in[x, x+a]}\|D f(t)-D f(x)\|
$$

Da continuidade uniforme de Df, temos:

$\forall \varepsilon>0, \quad \exists \delta>0 \quad$ tal que

$$
\|t-x\|<\delta \Rightarrow\left\|D f(t)-D_{f}(x)\right\|<\varepsilon \quad \forall t, x \in U
$$

Entã॰

$$
\begin{aligned}
& \|a\|<\delta \Longrightarrow\|t-x\|<\delta, \quad \forall t \in[x, x+a], \forall x \in U \Longrightarrow \\
& \Longrightarrow\|D f(t)-D f(x)\|<\varepsilon, \forall t \in[x, x+a], \forall x \in U \\
& \Longrightarrow \sup _{t \in[x, x+a]}\|D f(t)-D f(x)\|<\varepsilon, \quad \forall x \in U \\
& \Longrightarrow \frac{\|r(x, a)\|}{\|a\|}<\varepsilon, \quad \forall x \in U
\end{aligned}
$$

2.13 PROPOSIÇAO. Sejam E e F espaços normados e $f: E(r) \longrightarrow F$ uma função diferenciável. Definimos $f^{*}:\left(E \times E(r) \longrightarrow F \times F\right.$ por $\quad f^{*}(x, y)=(f(x), D f(x) y)$ Então: $\quad f^{*} \in U \mathrm{UC}^{\mathrm{k}-1} \Longleftrightarrow f \in U \mathrm{C}^{\mathrm{k}}, \quad \mathrm{k} \geqslant 1$. Demonstração: 
Ponhamos $f^{*}=(g, h)$, isto é, sejam $g$ e $h$ as compo nentes de $f^{*}$ no espaço produto $F \times F$; então: $g:(\mathbb{E} \times \mathbb{E})(r) \longrightarrow F$ e $h:(\mathbb{E} \times E)(r) \longrightarrow F$ são tais que $g(x, y)=f(x) \quad e$ $h(x, y)=\operatorname{Df}(x) y$.

Suponhamos em primeiro lugar que $f \in U C^{k}, \quad k \geqslant l$, e provemos que $f^{*} \in U \mathrm{U}^{\mathrm{k}-1}$. Esuficiente demonstrar que $g$ e $h$ são uniformemente $C^{k-1}$, uma vez que

$$
D^{p_{f}^{*}}=\left(D^{p}, D^{p_{h}}\right), \quad \forall p \in \mathbb{N} \text {. }
$$

Ora, é claro que $g=f 0 \pi_{I}$, onde $\pi_{l}$ é a primeira proje ção. Como $f \in U C^{k}$ e $\pi_{I} \in U C^{\infty}$, segue de 2.8 que g é uniformemente $\mathrm{c}^{\mathrm{k}}$. Quanto a $\mathrm{h}$, temos:

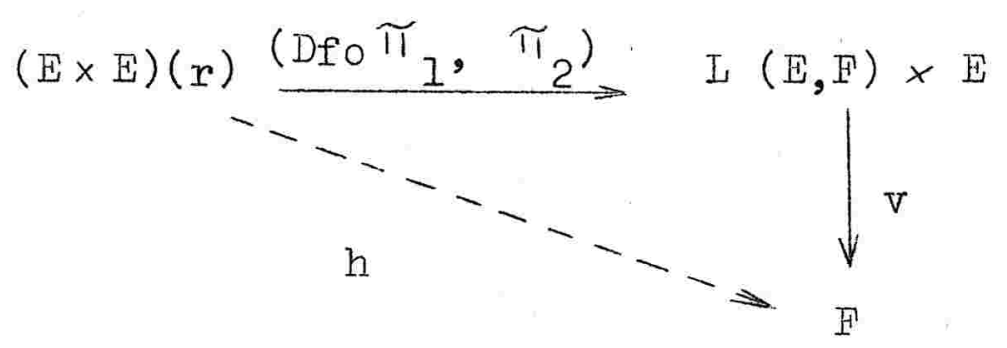

Então, $h \in U C^{k-1}$, pois $v \in U C^{\infty}$ ( proposiç̃o 2.11),

$$
\text { Df } \in U C^{k-1} \quad \text { e } \pi_{i} \in U C^{\infty} \quad(i=1,2)
$$

Demonstremos a recíproca por indução sôbre p. Para $\mathrm{p}=0$, temos da hipótese:

$\forall \varepsilon>0, \exists \delta>0$ tal que $\max \left\{\left\|x-x^{8}\right\|,\left\|y-y^{\prime}\right\|\right\}<\delta$ 
$\Longrightarrow \max \left\{\left\|f(x)-f\left(x^{\prime}\right)\right\|,\left\|D f(x) y-D f\left(x^{\prime}\right) y^{\prime}\right\|\right\}<r \varepsilon$

Então, para todo $y \in \mathbb{E}(r)$ :

$$
\begin{gathered}
\left\|x-x^{9}\right\|<\delta \Rightarrow\left\|(x, y)-\left(x^{9}, y\right)\right\|<\delta \Rightarrow\left\|D f(x) y-D f\left(x^{9}\right) y\right\|<r \varepsilon \\
\Longrightarrow\left\|\left[D f(x)-D f\left(x^{p}\right)\right] y\right\|<r \varepsilon
\end{gathered}
$$

Concluímos que

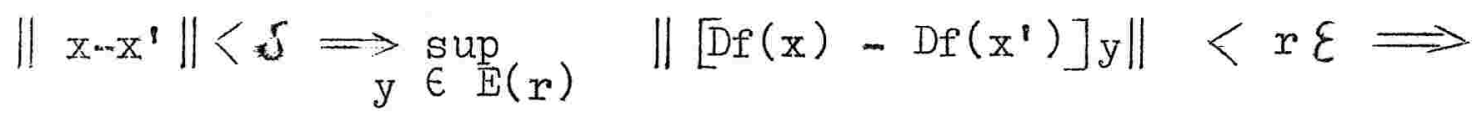

$$
\begin{aligned}
& \Longrightarrow r \cdot\left\|\mathrm{Df}(\mathrm{x})-\mathrm{Df}\left(\mathrm{x}^{\prime}\right)\right\|<r \varepsilon \Longrightarrow\left\|\mathrm{Df}(\mathrm{x})-\mathrm{Df}\left(\mathrm{x}^{\prime}\right)\right\|<\varepsilon
\end{aligned}
$$

Suponhamos agora válida a recíproca para $\mathrm{p}-1 \geqslant 0$ e que $f^{*} \in U_{C}{ }^{p}$. Intão, h é uniformemente ${ }_{C^{p}}$. Para aliviar a no tação, poremos. Df $=u$. Miostremos que u é diferenciável, provan do que

$$
D u(x)(a) y=D h(x, y)(a, 0), \forall x, y \in E(r), \forall a \in E
$$

Indiquemos então por $I_{X}$ a aplicação linear $E \rightarrow I(E, F)$ tal que $I_{x}(a) y=\operatorname{Dh}(x, y)(a, 0), \quad \forall a, y \in E, \quad\|y\|<r$ Então: para $x, a \in E(r)$ tais que $x+a \in E(r)$, temos: 
$\frac{2}{r} \sup _{r}\left\|u(x+a) y-u(x) y-I_{x}(a) y\right\|=$ $r\|\mathrm{y}\|<\mathrm{r}$

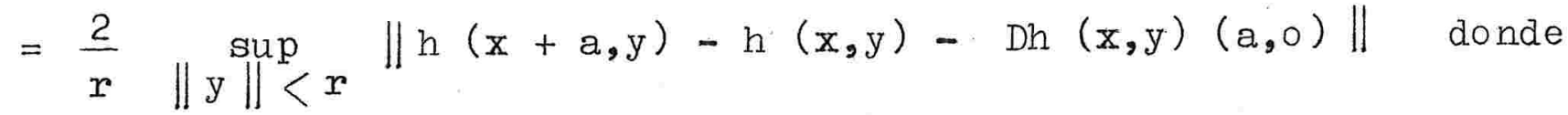

$$
\frac{\|r(x, a)\|}{\|a\|} \leqslant \frac{2}{r} \quad\|y\|<r\left\{\frac{1}{\|a\|}\|h(x+a, y)-h(x, y)-\operatorname{Dh}(x, y)(a, 0)\|\right\}
$$

Como $h \in C^{1}$, podemos, graças à proposição 2.12 , afị mar que a expressão entre chaves converge para zero com

$\|(a, 0)\|=\|a\|$, uniformemente em ( $x, y)$, e que por tanto $\forall \varepsilon>0, \exists \delta>0$ tal que $\|a\|<\delta \Longrightarrow\|r(x, a)\|<\varepsilon$. Isso nos mostra que $\mathrm{Du}(\mathrm{x})=\mathrm{I}_{\mathrm{x}}$, ou seja, $\Gamma u(x)(a) y=\operatorname{Dh}(x, y)(a, 0)$. Lembrando a identificação canôni ca de $L(E, I(E, F))$ com $I^{2}(E, F)$ e a simetria da derivada $D u(x)=D^{2} f(x)$, podemos escrever $\operatorname{Diu}(x),(y) a=D h(x, y)(a, 0): \forall x, y \in E(r), \forall a \in E$ ou $\operatorname{Du}(\mathrm{x}) \mathrm{y}=\operatorname{Dh}(\mathrm{x}, \mathrm{y}) \circ \mathrm{j}, \forall \mathrm{x}, \mathrm{y} \in \mathrm{E}(\mathrm{r})$, onde $j: E \longrightarrow E \times E$ é a inclusão $j(a)=(a, 0) \quad \forall a \in E$.

Definamos agora $\theta: L(E \times E, F) \longrightarrow L(E, F)$ por $\theta(u)=u \circ j$. É claro que $\theta$ é linear e contínua, e que por tanto $\theta \in U_{C^{\infty}}$. Além disso, para $x, y \in E(r)$, tem - se $u^{*}(x, y)=(u(x), D u(x) y)=\left(u \circ \pi_{1}(x, y), \theta \circ \operatorname{Dh}(x, y)\right)$

Isso mostra que $u^{*}=\left(u \circ \pi_{1}, \theta \circ\right.$ Dh $)$ e que portan to, pela proposição 2.8, $u^{*} \in \cup \mathrm{c}^{\mathrm{p}-1}$, uma vez que Dh $\in \cup \mathrm{C}^{\mathrm{p}-1} ; \theta, \pi_{1} \in U \mathrm{C}^{\infty}$ e $u \in \cup \mathrm{C}^{\mathrm{p}-1}$ (pois 
$f^{*} \in U \mathrm{U}^{\mathrm{P}} \Longrightarrow \mathrm{f}^{*} \in \mathrm{U} \mathrm{C}^{\mathrm{p}-1}$ e pela hipótese de indução isso a carreta $f \in U C^{p}$, donde $D f \in U C^{p-1}$ ). Agora, pela hipótese de indução, temos $u=D f \in U C^{p}$, e portanto $f \in U C^{p+I}$. $* * *$

2.14 PROPOSIÇÃO . Com as notações da proposição ante rior, se $f^{*} \in C^{l}$, temos:

$D\left(f^{*}\right)=\left(\theta_{1} \circ \mathrm{Df} \circ \pi_{1}, \theta_{2} \circ \mathrm{Df} \circ \pi_{1}+\theta_{1} \circ \mathrm{V} \circ\left(D^{2} f \circ \pi_{1}, \pi_{2}\right)\right)$ onde $\theta_{i}: L(E, F) \longrightarrow I(E x E, F)$ é definida por

$\theta_{i}(u)=u \circ \pi_{i}, \pi_{i}$ sendo a projeção de $E \times \mathbb{E}$ sôbre 0 i-ési mo fator $(i=1,2)$.

Demonstração :

Como $f^{*}=\left(f \circ \pi_{I}, V \circ\left(D f \circ \pi_{I}, \pi_{2}\right)\right)$, temos:

$D\left(f^{*}\right)(x, y)=\left(\operatorname{Df}(x) \circ \pi_{I}, \operatorname{Dv}(\operatorname{Df}(x), y) \circ\left(D^{2} f(x) \circ \pi_{1}, \pi_{2}\right)\right)$

Aplicando então o teorema 2.10 , obtemos, para $a, b \in E$ :

$D\left(f^{*}\right)(x, y)(a, b)=\left(\operatorname{Df}(x) a, D v(D f(x), y)\left(D^{2} f(x) a, b\right)\right)=$ $=\left(D f(x) a, D f(x) b+D^{2} f(x) a \cdot y\right)=\left(D f(x) a, D f(x) b+D^{2} f(x) y a\right)$. Logo, $D\left(f^{*}\right)(x, y)=\left(D f(x) \circ \pi_{I}, D f(x) \circ \pi_{2}+D^{2} f(x) y \circ \pi_{I}\right)$ donde

$D\left(f^{*}\right)=\left(\theta_{1} \circ D f \circ \pi_{1}, \theta_{2} \circ D f \circ \pi_{1}+\theta_{1} \circ \mathrm{V} \circ\left(D^{2} \perp \circ \pi_{1}, \pi_{2}\right)\right)$ 


\section{OPERADORES HIPERBÓIICOS}

3.I DEFINIÇÃO . Seja E um espaço de Banach. Um opera dor linear $T: E \rightarrow E$ é hiperbólico se $T$ é um isomorfismo (topo lógico) e se seu espectro (após complexificação) não contém elemen tos de modulo 1 . Demonstra-se em [9] que se $T$ é um operador hiperbólico, então existe uma única decomposição - dita canôni ca- $E=E_{1} \odot E_{2}$ om soma direta topológica com $E_{1} \odot E_{2}$ in variantes por. $T$, tal que o espectro de $T_{1}=T / E_{1}$ só contémpon tos exteriores e o espectro de $\mathrm{T}_{2}=\mathrm{T} / \mathrm{E}_{2}$ só contém pontos interiores à bola unitéria do plano complexo (ver também [10]). Além disso, podemos tomar normas em $E_{1} e E_{2}$, tais que a norma produto em $E$ seja equivalente à norma original, e tais que

$\left\|\mathrm{T}_{2}\right\|<1 \quad 0 \quad\left\|\mathrm{~T}_{I}^{-1}\right\|<1$ (ver [7]). No que segue, su poremos sempre $\mathrm{E}$ com a decomposição canônica, $\mathrm{E}_{1}$ e $\mathrm{E}_{2}$ com es sas normas, e E com a norma produto

$\|(x, y)\|=\max \{\|x\|,\|y\|\}$. Observe-se que em re lação a essa norma as projeções $\pi_{1}: E \rightarrow E_{1}$ e $\pi_{2}: E \rightarrow E_{2}$ têm norma 1 e que se $x=\left(x_{1}, x_{2}\right) \in E$ então $\pi_{i} \circ T(x)=T_{i}\left(x_{i}\right)(i=1,2)$.

Ao número $\zeta=\max .\left\{\left\|T_{2}\right\|,\left\|T_{1}^{-1}\right\|\right\}<I$ damos - nome de assimetria de T . Até o fim desta secção, E indicará um espaço de Banach, $T: E \longrightarrow E$ um operador hiperbólico com assimetria $\zeta$, e $f: E(r) \longrightarrow E$ uma função tal que $I(f-T)<\varepsilon<\frac{1-\zeta}{I+\zeta}$. Indicaremos ainda por $f_{1}$ e $f_{2}-$ 
33

as componentes de $f$ segundo a decomposição canônica de $E$, is to é, $f_{i}=\pi_{i} \circ f(i=1,2) \in f=\left(f_{1}, f_{2}\right)$. Veremos que nessas condições, se $f$ fôr inversível, seu comportamento será bas tante semelhante ao de $T$, isto é, existirão subconjuntos $W_{I}$ e $W_{2}$ invariantes por $f$ e tais que $f / W_{1}$ é uma expansão, enquanto que $f / W_{2}$ é uma contração. Notemos que, de $\varepsilon<\frac{1-\zeta}{1+\zeta}$, segue-se que $\varepsilon<\zeta^{-1}, \zeta^{-1}-\varepsilon>1, \varepsilon+\zeta<1$ e $\frac{\sigma+\varepsilon \cdot}{1-\zeta \varepsilon}<1$

3.2 LEMA - (a) $f_{2}$ é uma contração, com $I\left(f_{2}\right) \leqslant \zeta+\varepsilon$

(b) Se $x=\left(x_{1}, x_{2}\right)$ e $y=\left(y_{1}, y_{2}\right)$ são dois pontos de $E(r)$ e se $\left\|x_{1} \cdots y_{1}\right\| \geqslant\left\|x_{2}-y_{2}\right\|$ s então

$$
\left\|f_{1}(x)-f_{1}(y)\right\| \geqslant\left(\zeta^{-1}-\varepsilon\right)\left\|x_{1}-y_{1}\right\| \geqslant(\zeta+\varepsilon)\left\|x_{1}-y_{1}\right\| \geqslant\left\|f_{2}(x)-f_{2}(y)\right\|
$$

(c) Com as mesmas notações do item (b), se

$$
\left\|x_{1}-y_{1}\right\| \leqslant\left\|x_{2}-y_{2}\right\| \text {, então }\left\|f_{2}(x)-f_{2}(y)\right\|<(\zeta+\varepsilon)\left\|x_{2}-y_{2}\right\| \text {. }
$$

Demonstração :

$$
\begin{aligned}
& \text { (a) }\left\|f_{2}(x)-f_{2}(y)\right\|=\left\|\pi_{2} \circ f(x) \cdots \pi_{2} \circ f(y)\right\|= \\
& =\left\|\pi_{2}[(f-T)(x)-(f-T)(y)]+\pi_{2} \circ T(x-y)\right\| \leqslant \\
& \leqslant I(f-T)\|x-y\|+\left\|T_{2}\left(x_{2}-y_{2}\right)\right\|<\varepsilon\|x-y\|+\left\|T_{2}\right\|\left\|x_{2}-y_{2}\right\| \\
& \leqslant\left(\varepsilon+\left\|T_{2}\right\|\right)\|x-y\| \leqslant(\varepsilon+z)\|x-y\| .
\end{aligned}
$$


(b) Lembrando que $\left\|x_{1}-y_{1}\right\| \geqslant\left\|x_{2}-y_{2}\right\| \Longrightarrow\left\|x_{1}-y_{1}\right\|=\|x-y\|$ te mos: $\left\|f_{I}(x)-f_{I}(y)\right\|=\left\|\pi_{I} \circ f(x)-\pi_{I} \circ f(y)\right\|=$ $=\left\|\pi_{I}[(f-T)(x)-(f-T)(y)]+\pi_{I} \circ T(x-y)\right\| \geqslant$ $\geqslant\left\|\pi_{I} \circ T(x-y)\right\|-\left\|\pi_{I}[(f-T)(x)-(f \ldots T)(y)]\right\| \geqslant$ $\geqslant \| T_{1}\left(x_{1}-y_{1}\|-\|(f-T)(x)-(f-T)(y) \| \geqslant\right.$ $\geqslant\left\|\mathbb{T}_{1}^{-1}\right\|^{-1} \cdot\left\|x_{1}-y_{1}\right\|-I(f-T)\|x-y\|>\left(\zeta^{-I}-\varepsilon\right)\left\|x_{1}-y_{1}\right\|$ A segunda desi gualdade é consequência de

$$
\varepsilon+\zeta<1<z^{-1}-\varepsilon \text {. }
$$

Finalmente, de (a) segue-se que

$\left\|f_{2}(x)-f_{2}(y)\right\|<(\zeta+\varepsilon) \quad\|x-y\|=(\zeta+\varepsilon)\left\|x_{1}-y_{1}\right\|, 0$ que termina a demonstração de (b).

(c) Segue trivialmente de (a).

NOTAGÃO . Se $f$ é inversível, poremos para todo $n \in \mathbb{N}$ : $f^{n}(E(r))=\left\{x \in E(r): f^{-1}(x), f^{-2}(x), \ldots, f^{-n}(x)\right.$ estão de finidos e pertencem a $E(r)\}$.

$f^{-n}(E(r))=\left\{x \in \mathbb{E}(r): f(x), f^{2}(x), \ldots, f^{n}(x)\right.$ estão definidos $e$ pertencem a $E(r)\}$. Poremos ainda:

$W_{1}=n \geqslant 0 \quad f^{n}(E(r)) \quad e \quad W_{2}={ }_{n \geqslant 0}^{n} f^{-n}(E \cdot(r))$.

3.3 PROPOSIÇ̃̃O . W $W_{I}$ é o gráfico de uma função 
$\mathrm{U}_{1} \rightarrow \mathrm{E}_{2}(r)$ e $W_{2}$ é o gráfico de uma função $\mathrm{U}_{2} \rightarrow \mathrm{E}_{1}(r)$, on de $U_{1} \odot U_{2}$ são subconjuntos de $E_{1}(r)$ e $E_{2}(r)$, respectiva mente (supondo, é claro, f inversível, o que ocorre por exemplose $\mathcal{E}<\left\|T^{-1}\right\|^{-1} ;$ ver teorema 1.10$)$.

Demonstração .

Em primeiro lugar, consideremos o caso de $w_{2}$. É sufi ciente mostrar que se $z=\left(x_{1}, x_{2}\right)$ e $y=\left(y_{1}, y_{2}\right)$ são dois pon tos de $W_{2}$, com $x_{2}=y_{2}$, então $x_{1}=y_{1}$. Ora,

$x, y \in W_{2} \Longrightarrow f^{n}(x), f^{n}(y) \in \mathbb{E}(r), \forall n \in \mathbb{N}$. Aplicando então a parte (b) do lema 3.2 , temos :

$2 r>\left\|f^{n}(x)-f^{n}(y)\right\| \geqslant\left\|\pi_{I} \circ f^{n}(x)-\pi_{I} \circ f^{n}(y)\right\|=$

$=\left\|f_{1}\left(f^{n-1}(x)\right)-f_{1}\left(f^{n-1}(y)\right)\right\| \geqslant\left(\zeta^{-1}-\varepsilon\right)\left\|f_{I}^{n-1}(x)-f_{1}^{n-1}(y)\right\| \geqslant$

$\geqslant \cdots \geqslant\left(\zeta^{-1}-\varepsilon\right)^{n}\left\|x_{1}-y_{1}\right\|$. Logo, $\left\|x_{1}-y_{I}\right\|<\frac{2 r}{\left(\sigma^{-1}-\varepsilon\right)^{n}} \rightarrow 0$, pois $\zeta^{-1}-\varepsilon>1$. Concluímos que $x_{1}=y_{1}$.

Mostremos agora que se $x=\left(x_{1}, x_{2}\right)$ e $y=\left(y_{1}, y_{2}\right)$ são dois pontos de $W_{1} \operatorname{com} x_{1}=y_{1}$, então $x_{2}=y_{2}$. Dado $n>0$, ponhamos $x^{\prime}=f^{-n}(x)$ e $y^{\prime}=f^{-n}(y)$. De $x, y \in W_{1}$ segue -se que $x^{\prime}, y^{\prime} \in E(r)$. Temos aqui dois casos a considerar :

[A] Existe $j \in \mathbb{N}, 0 \leqslant j<n$, tal que

$\left\|f_{1}^{j}\left(x^{\prime}\right)-f_{1}^{j}\left(y^{\prime}\right)\right\| \geqslant\left\|f_{2}^{j}\left(x^{\prime}\right)-f_{2}^{j}\left(y^{\prime}\right)\right\|$ 
36

[B] $\left\|f_{1}^{j}\left(x^{p}\right)-f_{1}^{j}\left(y^{1}\right)\right\|<\left\|f_{2}^{j}\left(x^{p}\right)-f_{2}^{j}\left(y^{p}\right)\right\|$, $\forall j \in\{0,1, \ldots, n-1\}$

Caso $[\mathrm{A}]$ : Pelo lema 3.2, parte (b), temos:

$\left\|f_{1} \circ f^{j}\left(x^{1}\right)-f_{1} \circ f^{j}\left(y^{p}\right)\right\| \geqslant\left\|f_{2} \circ f^{j}\left(x^{1}\right)-f_{2} \circ f^{j}\left(y^{3}\right)\right\|$ ou $\left\|f_{1}^{j+1}\left(x^{1}\right)-f_{1}^{j+1}\left(y^{1}\right)\right\| \geqslant\left\|f_{2}^{j+1}\left(x^{p}\right)-f_{2}^{j+1}\left(y^{p}\right)\right\|$. Aplicando sucessivamente o lema, chegamos a

$$
\begin{aligned}
& \left\|f_{1}^{n}\left(x^{p}\right)-f_{1}^{n}\left(y^{\prime}\right)\right\| \geqslant\left\|f_{2}^{n}\left(x^{p}\right)-f_{2}^{n}\left(y^{p}\right)\right\| \text { ou seja } \\
& \left\|x_{1}-y_{1}\right\| \geqslant\left\|x_{2}-y_{2}\right\| \text { donde } x_{2}=y_{2} \text {. }
\end{aligned}
$$

Caso $[\mathrm{B}]$ : Pela parte (c) do Iema 3.2, temos agora

$$
\begin{aligned}
& \left\|x_{2}-y_{2}\right\|=\left\|f_{2}^{n}\left(x^{\prime}\right)-f_{2}^{n}\left(y^{\prime}\right)\right\|=\left\|f_{2} 0 f^{n-1}\left(x^{\prime}\right)-f_{2} 0 f^{n-1}\left(y^{\prime}\right)\right\|< \\
& <(\zeta+\varepsilon)\left\|f_{2}^{n-1}\left(x^{\prime}\right)-f_{2}^{n-1}\left(y^{\prime}\right)\right\|<\cdots<(\zeta+\varepsilon)^{n}\left\|x_{2}^{\prime}-y_{2}^{\prime}\right\|< \\
& <2 r(\zeta+\varepsilon)^{n} \rightarrow 0 \text { pois } \zeta+\varepsilon<1
\end{aligned}
$$

Conclusão: $\quad x_{2}=y_{2}$

Lembremos que $G=\left\{g \in\left(E_{1}(r), E_{2}(r)\right): I(g) \leqslant I\right\}$ é um espaço métrico completo com a distância dada por

$$
d\left(g, g^{\prime}\right)=\sup _{x \in \mathrm{E}_{1}(r)}\left\|g(x)-g^{\prime}(x)\right\|
$$

Para tôda função $g \in G$, poremos :

$$
\psi_{f}(g)=f_{I} \circ(I, g) \text { e } \varphi_{f}(g)=f_{2} \circ(I, g) \text { onde }
$$

$I: E_{I}(r) \longrightarrow E_{I}(r)$ é a aplicação idêntica. Então : 
$\psi_{f}(g): E_{1}(r) \rightarrow E_{1}$ e $\varphi_{f}(g): E_{1}(r) \rightarrow E_{2}$.

3.4 PROPOSIÇÃO . (a) $\psi_{f}(g)$ é um homeomorfismo de $E_{I}(r)$ sôbre um aberto de $E_{j}$ e $\psi_{f}(g)^{-1}$ é uma contração.

(b) Se $f(0)=0$, então $E_{I}(r) \subset \psi_{f}(g)\left(E_{I}(r)\right)$ e portanto $\psi_{f}(g)^{-1}: E_{I}(r) \rightarrow E_{I}(r)$ está bem definida.

(c) $\varphi_{f}(g)$ é uma contração.

Demonstração .

(a) Se $x, y \in E_{1}(r)$, temos:

$\left\|\left(\psi_{f}(g)-T_{I}\right)(x)-\left(\psi_{f}(g)-T_{I}\right)(y)\right\|=$

$=\left\|\pi_{I} \circ f(x, g(x))-\pi_{I} \circ T(x, g(x))-\pi_{I} \circ f(y, g(y))+\pi_{I} \circ T(y, g(y))\right\|$

$=\left\|\pi_{I}[(f-T)(x, g(x))-(f-T)(y, g(y))]\right\| \leqslant$

$\leqslant I(f-T)\|(x-y, g(x)-g(y))\|=I(f-T) .\|x-y\|$ (pois

$I(g) \leqslant 1 \Longrightarrow\|x-y\| \geqslant\|g(x)-g(y)\|)$. Então, $\psi_{f}(g)-T_{I}$ é lipschitziana e $L\left(\psi_{f}(g)-T_{I}\right) \leqslant L(f-T)<\varepsilon<\zeta^{-1} \leqslant\left\|T_{I}^{-1}\right\|^{-1}$. Pelo teorema 1.10 , concluímos que $\psi_{f}(g)$ é um homeomorfismo de $\mathrm{E}_{1}(r)$ sôbre um aberto de $\mathrm{E}_{1}$. Além disso, temos $I\left(\psi_{f}(g)^{-1}\right) \leqslant\left[\left\|T_{I}^{-1}\right\|^{-I}-I\left(\psi_{f}(g)-T_{I}\right)\right]^{-1} \leqslant\left(\zeta^{-1}-\varepsilon\right)^{-I}<I$ (b) Se $f(0)=0$, temos $\psi_{f}(g)(0)=0$, e como $\psi_{f}(g)$ sati $\underline{\underline{s}}$ 
faz às condiçôes do teorema 1.10 , segue-se de 1.11 que

$E_{I}(r) \approx \psi_{f}(g)\left(E_{I}(r)\right):$ basta tomar $\tilde{r}$ tal que $\tilde{r}<r<\left(\zeta^{-1}-\varepsilon\right) \tilde{r}$ e teremos (por 1.11)

$\mathrm{E}_{1}(r) \subset \mathrm{E}_{1}\left(\left(\zeta^{-1}-\varepsilon\right) \tilde{r}\right) \subset \mathrm{E}_{1}\left(\left[\left\|\mathrm{~T}_{I}^{-1}\right\|^{-1}-I_{(}\left(\psi_{f}(g)-\mathrm{T}_{1}\right)\right] \tilde{r}\right) \subset$ $E \psi_{f}(g)\left(E_{1}(r)\right)$.

(c) Sendo $f_{2}$ uma contração (lema 3.2) e ( $($, g ) uma isometria, segue-se pela proposiç̃o 1.6 que $I\left(\varphi_{f}(g)\right) \leqslant L\left(f_{2}\right) \cdot I(I, g)<I$ ***

3.5 DEFINIÇÃO. Suponhamos que $f(0)=0$. A transformação - gráfico associada a $f$ é a aplicação $\Gamma_{f}: G \longrightarrow G$ de finida por $\Gamma_{f}(g)=f_{2} \circ(I, g) \circ\left[f_{I} \circ(I, g)\right]^{-1} / \mathrm{I}_{I}(r)$ ou seja, $\Gamma_{f}(g)=\varphi_{f}(g) \circ \psi_{f}(g)^{-I} / E_{I}(r)$, A proposição an terior assegura que o segundo membro faz sentido e que realmente $\Gamma_{f}(g) \in G, \forall g \in G$. Mais ainda, $I\left(\Gamma_{f}(g)\right)<I, \forall g \in G_{0}$ o têrmo "transformação - gráfico" se justifica pela

$$
\text { 3.6 PROPOSICUAO } h=\Gamma_{f}(g) \text { se, e sòmente se, }
$$
$f($ graf $g) \cap E(r)=$ graf $h$.

Demonstração .

Suponhamos que $h=\Gamma_{f}(g)$. Então, se (x, y) $\in$ graf $h \subset \mathrm{h}(\mathrm{r})$, temos $\mathrm{y}=\mathrm{h}(\mathrm{x})$, isto é 


$$
y=f_{2^{\circ}}(I, g) \circ\left[f_{I^{\circ}}(I, g)\right]^{-I}(x)
$$

IIIa.s $x \in E_{I}(r) \Longrightarrow u=\left[f_{I^{\circ}}(I, g)\right]^{-1}(x) \in E_{I}(r) \quad(\operatorname{ver} 3.4)$

Logo, $\quad y=f_{2}(u, g(u))$ e $x=f_{1}(u, g(u))$, donde

$$
(x, y)=\left(f_{I}(u, g(u)), f_{2}(u, g(u))=f(u, g(u)) \in f(\operatorname{graf} g)\right.
$$

e portanto graf $h \subset f($ graf $g) \cap E(r)$.

Por outro lado, se $(x, y) \in f($ graf $g) \cap E(r)$, temos

$$
(x, y)=f(v, g(v)), \text { com } v \in \mathbb{F}_{1}(r), \text { e portanto }
$$

$$
\begin{aligned}
& x=f_{1}(v, g(v))=f_{1} \circ(I, g)(v) \\
& y=f_{2}(v, g(v))=f_{2} \circ(I, g)(v)
\end{aligned}
$$

Segue-se que

$$
v=\left[f_{1} \circ(I, g)\right]^{-1}(x)
$$

e portanto

$$
y=f_{2} \circ(I, g) \circ\left[\left(f_{1} \circ(I, g)\right]^{-\cdots]}(x)=h(x)\right.
$$

Conclusão: $\quad(\mathrm{x}, \mathrm{y}) \in$ graf $h$.

Então: $f($ graf $g) \cap E(r) \subset g r a i h$.

Recìprocamente, se $f($ graf $g) \cap \mathbb{E}(r)=$ graf $h$, então, para todo $x \in E_{I}(r)$, 
40

pondo $\quad y=\left[f_{I} \circ(I, g)\right]^{-1}(x), \quad$ temos:

$$
\Gamma_{f}(g)(x)=f_{2} \circ(1, g) \circ\left[f_{1} \circ(I, g)\right]^{\cdots}(x)=f_{2}(y, g(y))
$$

Logo, $\left(x, \Gamma_{f}(g)(x)\right)=\left(f_{1} \circ(1, g)(y), f_{2}(y, g(y))\right)=$

$$
=f(y, g(y)) \in f(g r a f g)
$$

Também $\left(x, \Gamma_{f}(g)(x)\right) \in E(r)$, pois $I\left(\Gamma_{f}(g)\right)<1 \quad$ e $\|x\|<r$. Então, $\left(x, \Gamma_{f}(g)(x)\right) \in f(g r a f g) n E(r)=\operatorname{graf} h$.

- que prova que

$$
h(x)=\Gamma_{f}(g)(x) .
$$

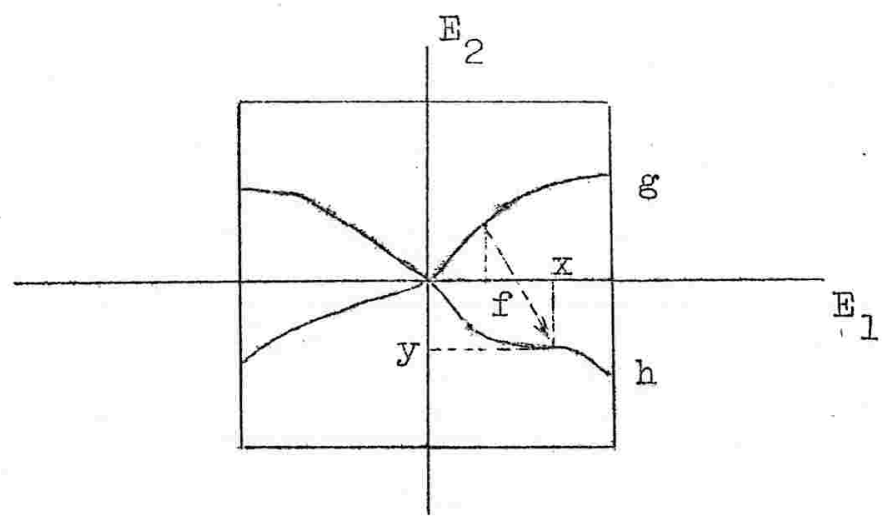

3.7 OBSERVACRO. Tomando $\mathrm{f}=\mathrm{T} / \mathrm{E}(\mathrm{r})$, teremos

$$
\Gamma_{\mathrm{T}}(\mathrm{g})=\mathrm{T}_{2} \circ \mathrm{g} \circ \mathrm{T}_{1}^{-1} / \mathrm{E}_{\mathrm{I}}(\mathrm{r}), \forall \mathrm{g} \in \mathrm{G} \text {. }
$$

De fato, basta notar que $\tilde{\pi}_{1}$ oT $O(l, g)=T_{1}$

e

$\pi_{2} \circ \mathrm{OT}(I, g)=T_{2} \circ g$ ( atentemse para 0 fato de que as componentes 
de $f$ são $\pi_{1} \circ \mathrm{T} / \mathrm{E}(\mathrm{r})$ e $\pi_{2} \circ \mathrm{T} / \mathrm{E}(\mathrm{r})$, e não $\mathrm{T}_{1}$ e $\mathrm{T}_{2} !$ )

3.8 PRO POSI @̧̃O A transformação-gráfico $\Gamma_{f}$ é uma contração de $G$, e como $G$ é completo, existe um atrator $g_{f}$ para $\Gamma_{f}$, que é por ala vez uma contração.

Demonstração:

Para quaisquer $g_{1}, g_{2}, \in G$, temos pela proposição 1.6:

$d\left(\Gamma_{f}\left(g_{1}\right), \Gamma_{f}\left(g_{2}\right)\right)=d\left(\rho_{f}\left(g_{1}\right) \circ \psi_{f}\left(g_{1}\right)^{-1}, \rho_{f}\left(g_{2}\right) \circ \psi_{f}\left(g_{2}\right)^{-1}\right) \leqslant$

$\leqslant d\left(\varphi_{f}\left(g_{1}\right), \varphi_{f}\left(g_{2}\right)\right)+L\left(\varphi_{f}\left(g_{2}\right)\right) \cdot d\left(\psi_{f}\left(g_{1}\right)^{-1}, \psi_{f}\left(g_{2}\right)^{-1}\right) \leqslant$

$\leqslant d\left(\varphi_{f}\left(g_{1}\right), \varphi_{f}\left(g_{2}\right)\right)+I\left(\varphi_{f}\left(g_{2}\right)\right) \cdot I\left(\psi_{f}\left(g_{I}\right)^{-I}\right)_{0} d\left(\psi_{f}\left(g_{I}\right), \psi_{f}\left(g_{2}\right)\right) \leqslant$

$\leqslant a\left(\varphi_{f}\left(g_{1}\right), \varphi_{f}\left(g_{2}\right)+(\zeta+\varepsilon) \cdot\left(\zeta^{-1}-\varepsilon\right)^{\cdots-1} \cdot d\left(\psi_{f}\left(g_{1}\right), \psi_{f}\left(g_{2}\right)\right)\right.$

Mas: $\forall x \in E_{1}(r)$,

$\left\|\rho_{f}\left(g_{1}\right)(x)-\varphi_{f}\left(g_{2}\right)(x)\right\|=\left\|f_{2}\left(x, g_{1}(x)\right)-f_{2}\left(x, g_{2}(x)\right)\right\| \leqslant$

$\leqslant \| \pi_{2}\left[(f-T)\left(x, g_{1}(x)\right)-(f-T)\left(x, g_{2}(x)\right]\|+\| \pi_{2} 0 T\left(0, g_{1}(x)-g_{2}(x) \|\right.\right.$

$\leqslant L(f-T)\left\|g_{1}(x)-g_{2}(x)\right\|+\left\|T_{2}\right\| \cdot\left\|g_{1}(x)-g_{2}(x)\right\| \leqslant$ 
$<(\varepsilon+\zeta)\left\|g_{1}(x)-g_{2}(x)\right\|$

Daí, $\alpha\left(\varphi_{f}\left(g_{1}\right), \varphi_{f}\left(g_{2}\right)\right)=\sup _{x \in \mathbb{E}_{1}}\left\|_{r} \varphi_{f}\left(g_{1}\right)(x)-\rho_{f}\left(g_{2}\right)(x)\right\| \leqslant$

$\leqslant(\zeta+\varepsilon) \sup _{x \in \mathbb{E}_{1}(r)}\left\|g_{1}(x)-g_{2}(x)\right\|=(\zeta+\varepsilon) \cdot d\left(g_{1}, g_{2}\right)$

Por outro lado, $\forall x \in E_{1}(r)$ :

$\left\|\psi_{f}\left(g_{1}\right)(x)-\psi_{f}\left(g_{2}\right)(x)\right\| \leqslant$

$\leqslant\left\|\pi_{1}\left[(f-T)\left(x, g_{1}(x)\right)-(f-T)\left(x, g_{2}(x)\right)\right]\right\|+$

$+\left\|\pi_{1} \circ \mathrm{T}\left(0, g_{1}(x)-g_{2}(x)\right)\right\| \leqslant$

$\leqslant L(f-T) \quad\left\|g_{1}(x)-g_{2}(x)\right\|+\left\|T_{1}(0)\right\|<\varepsilon\left\|g_{1}(x)-g_{2}(x)\right\|$

Daí, $\quad a\left(\psi_{f}\left(g_{1}\right), \psi_{f}\left(g_{2}\right)\right)=\sup _{x \in \mathbb{E}_{1}(r)}\left\|\psi_{f}\left(g_{1}\right)(x)-\psi_{f}\left(g_{2}\right)(x)\right\| \leqslant$

$\leqslant \varepsilon \sup _{x \in \mathrm{F}_{1}(r)}\left\|g_{1}(x)-g_{2}(x)\right\|=\varepsilon \cdot d\left(g_{1}, g_{2}\right)$

( Mostramos que $\varphi_{f}: G \longrightarrow \mathbb{M}\left(E_{1}(r), E_{2}\right)$ e $\psi_{f}: G \rightarrow \mathbb{M}\left(E_{1}(r), E_{1}\right)$ sãc contrações $)$. 
Voltando à desigualdade inicial, temos:

$$
\begin{aligned}
& d\left(\Gamma_{f}\left(g_{1}\right), \Gamma_{f}\left(g_{2}\right)\right) \leqslant d\left(\varphi_{f}\left(g_{1}\right), \varphi_{f}\left(g_{2}\right)\right)+ \\
& +(\zeta+\varepsilon)\left(\zeta^{-1}-\varepsilon\right)^{-1} \cdot d\left(\psi_{f}\left(g_{1}\right), \psi_{f}\left(g_{2}\right)\right) \\
& \leqslant\left[(\zeta+\varepsilon)+(\zeta+\varepsilon) \cdot\left(\zeta^{-1}-\varepsilon\right)^{-1} \cdot \varepsilon\right] d\left(g_{1}, g_{2}\right)= \\
& =\frac{\zeta+\varepsilon}{1-\zeta \varepsilon} \cdot d\left(g_{1}, g_{2}\right)
\end{aligned}
$$

Logo, $\Gamma_{f}$ é uma contração. Finalmente, o atrator $g_{f}$ de $\Gamma_{f}$ é por vez uma contração, pois

$$
\Gamma_{f}\left(g_{f}\right)=g_{f} \Longrightarrow I\left(g_{f}\right)<I \quad(v \cdot 3.5)
$$




\section{OS TMOREIVIAS DAS VART EDADES}

IINSTAVEL E ESTAVEL

4.1 LEMA. Seja $E$ um espaço de Banach, $T: E \rightarrow E$ um operador hiperbolico com assimetria $\zeta, e f: E(r) \longrightarrow E$ uma função tal que

$$
I(f-T)<\varepsilon<\min \left\{\frac{1-b}{1+\sigma},\left\|T^{-1}\right\|^{-1}\right\}
$$

e $f(0)=0$. Então, existe uma (única) função $g_{f}: E_{I_{l}}(r) \rightarrow E_{2}(r)$ cujo gráfico é $W_{I}$ e tal que $\mathrm{I}\left(g_{f}\right)<I$ ( as notações são as mes mas que as da secção 3 ).

Demonstração :

Seja $g_{f}$ o atrator da transformação-gráfico $\Gamma_{f}: G \rightarrow G$ ( v. proposição 3.8). Como $g_{f}: E_{1}(r) \rightarrow \overline{E_{2}(r)}$ e $L\left(g_{f}\right)<I$ temos $g_{f}: E_{l}(r) \longrightarrow E_{2}(r)$. Niostremos que graf $g_{f}=W_{I}$, 直 suficiente mostrar que graf $g_{f}=W_{1}$, pois de 3.3 sabemos que $W_{1}$ é gráfico de uma função $U_{1} \longrightarrow E_{2}(r), \quad U_{I} \subset E_{1}(r)$. Em outras palavras, devemos provar que graf $g_{f} \subset f^{n}(E(r)), \forall n \in \mathbb{N}$. Proce deremos por indução: se $\mathrm{n}=0$, não há o quefazer, uma vez que $f^{0}(E(r))=E(r)$. Suponhamos que graf $g_{f} \subset f^{n-1}(E(r))$, para $n \geqslant 1$. Então: se $x \in \operatorname{gral} g_{f}=f\left(g r a f g_{f}\right) \cap E(r)$ (proposição $3.6)$, temos $x=f(y)$, com $y £ \operatorname{graf} g_{f} \subset f^{n-1}(E(r))$. Logo, $f^{-k}(y) \in E(r)$, para $k=1, \ldots, n-1$, donde $f^{-k}\left(f^{-1}(x)\right) \in E(r)$ 
para $1 \leqslant k \leqslant n-1$, e portanto $f^{-j}(x) \in E(r)$, para $j=2, \ldots, n$. Como $f^{-I}(x)=y \in \mathbb{E}(r)$, temos $f^{-j}(x) \in \mathbb{E}(r)$ para $I \leqslant j \leqslant n$, que garante que $z \in f^{n}(E(r))$.

Conclusão : graf $g_{f} \subset f^{n}(E(r))$.

4.2 LeMIA Nas condições do lema anterior, a aplicação $f^{-1 /} W_{1}: W_{I} \rightarrow W_{I}$ está bem definida e é uma contração. Demonstração:

De $P\left(\right.$ grai $\left.g_{f}\right) \cap \mathbb{E}(r)=$ graf $g_{P}$ segue-se que $f\left(W_{I}\right) \supset W_{I}$, o que dá sentido a $f^{-I} / W_{I} \rightarrow W_{I}$. De fato: se $x \in \mathbb{W}_{1}$, então $x \in f^{11}(E(r)), \forall n \in \mathbb{N}$, ou seja, $f^{-j}(x)$ existe e pertence a $\mathbb{E}(r), \forall j \in \mathbb{N}$. Sc ja então $y=f^{-1}(x)$. E claro que $y \in W_{I}$, pois $f^{-j}(y)=r^{-j-I}(x) \in E(r), \forall j \in \mathbb{W}$. Logo, $x=f(y) \in f\left(w_{\perp}\right)$. Mostre mos agora que $f^{-I} / W_{1}: W_{1} \longrightarrow W_{1}$ é uma contração. Inicialmente, é fácil ver que o diagrama

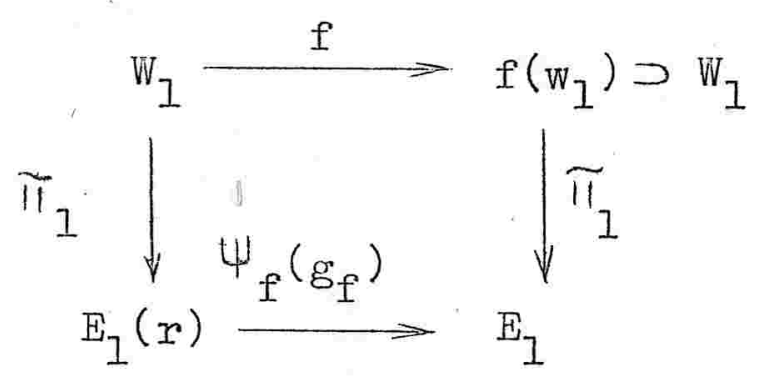

é comutativo. Seguerse daí que, para a $\in \mathrm{E}_{\mathbf{L}}(\mathrm{r})$, 


$$
\left(f^{-1}\right)_{I}\left(a, g_{f}(a)\right)=\psi_{f}\left(g_{f}\right)^{-1}(a) .
$$

Além disso, $I\left(g_{f}\right)<I$ acarreta que $\pi_{1}$-é uma isometria de $W_{1}$ sôbre $E_{I}(r)$, e portanto, se $u=\left(a, g_{f}(a)\right), v=\left(b, g_{f}(b) \in W_{I}\right.$, temos:

$$
\begin{aligned}
& \left\|f^{-I}(u)-f^{-I}(v)\right\|=\left\|f_{I}^{-I}\left(a, g_{f}(a)\right)-f_{I}^{-I}\left(b, g_{f}(b)\right)\right\|= \\
& =\left\|\psi_{f}\left(g_{f}\right)^{-I}(a)-\psi_{f}\left(g_{f}\right)^{-1}(b)\right\| \leqslant I\left(\psi_{f}\left(g_{f}\right)^{-1}\right) \quad\|a-b\|= \\
& =I\left(\psi_{f}\left(g_{f}\right)^{-1}\right) \quad\|u-v\| .
\end{aligned}
$$

Logo., $f^{-1} / W_{I}$ é uma contração, já que

$$
I\left(\psi_{f}\left(g_{f}\right)^{-1}\right) \leqslant\left(\xi^{-1}-\varepsilon\right)^{-1}<1
$$

4.3 COROLARTO, A origem é o único ponto fixa de $f$.

Demonstração :

Qualquer ponto fixo de $f$ deve pertencer a $W_{1}$, pois se $f(a)=a$, então $f^{-j}(a)=a \in E(r), \forall j \in \mathbb{N}$. Por outro lado, sen do $f^{-1} / W_{I}$ uma contração, $f^{-1}$ tem no máximo um ponto fixo em $W_{1}$ 
4.4 PROPOSI ÇAO. Se ja $f: E(r) \longrightarrow E$ como no lema 4.1 e suponhamos que $f \in U \mathrm{UC}^{\mathcal{I}}$. Então, $g_{f} \in U \mathrm{UC}^{\mathcal{I}}$.

Demonstrą̧̃o :

Como $T \in C^{\infty}$, temos $f-T \in C^{I}$ e portanto, pelo lema

1.9 ,

$$
\|D(f-T)(z)\| \leqslant L(f-T)<\varepsilon, \quad \forall z \in \mathbb{E}(r)
$$

Segue-se daí que

$$
\|D f(z)-T\|<\varepsilon, \quad \forall z \in \mathbb{E}(r)
$$

Isso quer dizer que, para todo $z \in E(r)$, a função $D f(z) / E(I)$ que indicaremos ainda por Df $(z)$ - é tal que

$$
I(D f(z)-T)=\|D f(z)-T\|<\varepsilon
$$

e portanto está bem definida a transformação-gráfico a ela associa da,

$$
\Gamma_{\operatorname{Df}(z)}: G_{1} \longrightarrow G_{1}
$$

onde $\quad G_{1}=\left\{g \in \mathbb{M}\left(E_{1}(I), \overline{\left.E_{2}(I)\right)}: L(g) \leqslant I\right\} ;\right.$

lembremos que, para $g \in G_{1}$,

$$
\Gamma_{D f(z)}(g)=\mathrm{Df}_{2}(z) \circ(I, g) \circ\left[\mathrm{Df}_{1}(z) \circ(I, g)\right]^{-1} / \mathrm{E}_{1}(I)
$$


- que $\Gamma_{\operatorname{Df}(z)}(g)$ é uma contração, $\forall g \in G_{1}$. Consideremos agora o espaço ( completo)

$$
H=\left\{h \in C^{0}\left(E_{1}(r), L\left(E_{1}, E_{2}\right)\right):\|h(x)\| \leqslant 1, \forall x \in E_{1}(r)\right\}
$$

que nada mais é do que a bola fechada unitária do espaço de Banach das funções contínuas e limitadas $E_{1}(r) \rightarrow I\left(E_{1}, E_{2}\right)$. Observe -se qle, quaisquer que sejam $h \in H \in z_{I} \in E_{I}(r)$, temos $\hbar\left(z_{l}\right)=h\left(z_{l}\right) / E_{I}(I) \in G$ e que portanto, se $z=(I, g)\left(z_{I}\right)$, então $\quad \prod_{\operatorname{Df}(z)}\left(\hbar\left(z_{1}\right) \in G_{1} \quad e\right.$

$$
\Gamma_{\operatorname{Df}(z)}\left(\hbar\left(u_{1}\right)\right)=D f_{2}(z) \circ\left(I, \hbar\left(z_{1}\right)\right) \circ\left[D f_{1}(z) \circ\left(I, \bar{h}\left(z_{1}\right)\right)\right]^{-1} / E_{1}(I)
$$

Definimos entẽo, para cada $g \in G$, a função

$$
\omega_{g}: H \rightarrow H
$$

tal que, $\forall \mathrm{h} \in \mathrm{H}$ :

$$
w_{g}(h)(x)=D f_{2}(z) \circ\left(I, h\left(z_{1}\right)\right) \circ\left[D f_{1}(z) \circ\left(I, h\left(z_{1}\right)\right)\right]^{-1}
$$

onde $\quad z_{1}=\psi_{f}(g)^{-1}(x), \quad z_{2}=g\left(z_{1}\right)$ e $z=\left(z_{1}, z_{2}\right)$. Em outras palavras, $w_{g}(h)(x)$ é a ( única ) extensäo linear a $E_{I}$ de 
$\Gamma \quad\left(\hbar\left(z_{1}\right)\right)$. Logo, está bem definida $c$ $\mathrm{Df}(\mathrm{z})$

$$
\left\|w_{g}(h)(x)\right\| \leqslant 1, \quad \forall x \in E_{1}(r)
$$

pois $W_{g}(h)(x)$, restrita à bola unitária, é uma contração. Isso mostra que $W_{g}(h) \in H$. Mostremos que $W_{g}$ é uma contração:

$$
a\left(w_{g}\left(h_{1}\right), w_{g}\left(h_{2}\right)\right)=\sup _{x \in E_{I}(r)}\left\|w_{g}\left(h_{1}\right)(x)-w_{g}\left(h_{2}\right)(x)\right\|=
$$

$=\sup _{X \in E_{I}(r)} d\left(\Gamma_{D f(z)}\left(\bar{h}_{1}\left(z_{I}\right)\right), \cdot \Gamma_{\left.\left.D f(z)^{\left(h_{2}\right.}\left(z_{1}\right)\right)\right)}\right.$

$\leqslant \sup _{x \in E_{1}(r)} \bar{K} \cdot d\left(\bar{h}_{1}\left(z_{1}\right), \bar{h}_{2}\left(z_{1}\right)\right)$

onde $\quad K=I(\Gamma \operatorname{Df}(z))<I \cdot \quad$ Mas

$$
x \in E_{I}(r) \Longrightarrow z_{I}=\psi_{f}(g)^{-I}(x) \in E_{I}(r) .
$$

Ingo,

$$
\begin{aligned}
& a\left(\omega_{g}\left(h_{1}\right), \omega_{g}\left(h_{2}\right)\right) \leqslant K \sup _{z_{1}} \operatorname{Ev}_{1}(r)\left\|h_{1}\left(z_{1}\right)-h_{2}\left(z_{1}\right)\right\|= \\
& =K d\left(h_{I}, h_{2}\right) \text {. }
\end{aligned}
$$

Construímos assim uma família $\left(\omega_{g}\right)_{g} \in G$ de contrações $\omega_{g}: H \longrightarrow H \cdot$ Seja 


$$
\mathrm{F}_{f}: \mathrm{G} \times \mathrm{H} \longrightarrow \mathrm{G} \times \mathrm{H}
$$

tal que

$$
F_{f}(g, h)=\left(\Gamma_{f}(g), \omega_{g}(h)\right) .
$$

Já sabemos que $\Gamma_{f}: G \rightarrow G$ tem um atrator $g_{f}$ : logo, para re cairmos nas condições do teorema da contração em fibras (1.8), bas ta mostrar que, para cada $h \in H$, a função $u: G \rightarrow H$ tal que

$$
u(g)=w_{g}(h)
$$

é contínua. Então: seja $g \in G$ e mostremos que u é contínua em g:

$$
\mathrm{d}\left(w_{g}(h), w_{\left.g^{\prime}(h)\right)}=\sup _{x \in E_{l}(r)}\left\|w_{g}(h)(x)-w_{g^{\prime}}(h)(x)\right\|\right.
$$

Seja $\quad A(x)=\left\|W_{g}(h)(x)-w_{g^{+}}(h)(x)\right\| \quad$ Temos

$$
\begin{aligned}
& A(x)=\| D f_{2}(z) \circ\left(I, h\left(z_{I}\right) \circ\left[D f_{I}(z) \circ\left(I, h\left(z_{I}\right)\right)\right]^{-1}\right. \\
& -D f_{2}\left(z^{p}\right) \circ\left(I, h\left(z_{1}^{p}\right)\right) \circ\left[D f_{1}\left(z^{p}\right) \circ\left(I, h\left(z_{l}^{p}\right)\right)\right]^{-1} \| \\
& \text { onde: } z=(1, g) \circ\left[f_{1} \circ(1, g)\right]^{-1}(x)=\left(z_{1}, z_{2}\right) \\
& z^{p}=\left(I, g^{\prime}\right) \circ\left[f_{1} \circ\left(I, g^{\prime}\right)\right]^{-1}(x)=\left(z_{1}^{1}, z_{2}^{p}\right)
\end{aligned}
$$


51

Como as funções envolvidas na expressão acima são tôdas Iineares, suas normas são suas constantes de Iipschitz. Aplicando então a proposição 1.6, obtemos:

$$
\begin{aligned}
& A(x) \leqslant\left\|D f_{2}(z)-D f_{2}\left(z^{i}\right)\right\|+ \\
& +\left\|\mathrm{Df}_{2}\left(z^{i}\right)\right\|\left\{\left\|h\left(z_{1}\right)-h\left(z_{1}^{\prime}\right)\right\|+L\left(\psi_{D f(z)}\left(\bar{h}\left(z_{1}\right)\right)^{-1}\right) .\right. \\
& \text { - } \left.\left[\|\|_{f_{I}}(z) \ldots D f_{I}\left(z^{\prime}\right)\|+\| D f_{I}\left(z^{\prime}\right)\|\quad\| h\left(z_{I}\right)-h\left(z_{1}^{\prime}\right) \|\right]\right\} \\
& \left(\text { note-se que }\left\|\left(I, h\left(z_{1}^{\prime}\right)\right)\right\|=1 \text {, pois }\left\|h\left(z_{1}^{i}\right)\right\| \leqslant 1\right. \text {. } \\
& \text { litas, para todo } x \in \mathrm{E}_{I}(n) \text { : } \\
& \left\|z-z^{\prime}\right\|=\left\|(I, g) \circ\left[f_{1} \circ(1, g)\right]^{\cdots I}(x)-\left(1, g^{8}\right) \circ\left[f_{1} \circ\left(1, g^{\prime}\right)\right]^{-1}(x)\right\| \leqslant \\
& \leqslant d\left((1, g) \circ\left[f_{1} \circ(1, g)\right]^{-1},\left(1, g^{n}\right) \circ\left[f_{1} \circ\left(1, g^{1}\right)\right]^{-1}\right) \leqslant \\
& \leqslant d\left(g, g^{\prime}\right)+I\left(\psi_{f}(g)^{-1}\right) \cdot d\left(\psi_{f}(g), \psi_{f}\left(g^{\prime}\right)\right) \\
& \text { Ora, } \quad I\left(\psi_{f}(g)^{-1}\right)<1 \text { e } \\
& d\left(\psi_{f}(g), \psi_{f}\left(g^{\prime}\right)\right)<\mathcal{E} \cdot d\left(g, g^{\prime}\right)
\end{aligned}
$$

(Como foi visto em 3.8). Segue-se que 
52

$\left\|z-z^{\prime}\right\| \leqslant(1+\varepsilon) d\left(g, g^{p}\right), \quad \forall x \in E_{l}(r)$.

Então, para todo $\rho>0$, existe $\rho_{1}>0$, independente de $x$, tal que

$$
d\left(g, g^{\prime}\right)<\rho_{I} \Rightarrow\left\|z-z^{\prime}\right\|<\rho
$$

Por outro lado, sendo Df uniformemente contínua em $\mathbb{E}(r)$, temos:

$V \eta>0, \exists \rho>0$ tal que

$$
\begin{gathered}
\left\|z-z^{1}\right\|<\rho^{*} \Longrightarrow\left\|D f(z)-D f\left(z^{8}\right)\right\|<\eta \Longrightarrow \\
\left\|D f_{i}(z)-D f_{i}\left(z^{1}\right)\right\|<\eta \quad(i=1,2)
\end{gathered}
$$

Além disso, h sendo continua, temos:

$\forall \eta>0, \exists \rho^{n} 0$ tal que $\left\|z-z^{\prime}\right\|<\rho^{\prime \prime} \Rightarrow\left\|h\left(z_{I}\right)-h\left(z_{l}^{\prime}\right)\right\|<\eta$

Finalmente, sabemos que existe $\mathbb{M}>0$ tal que

$\left\|D f_{i}\left(z^{p}\right)\right\| \leqslant \mathbb{M}, \quad \forall z^{\prime} \in \mathbb{E}(r) \quad(i=1,2) \quad$ ( basta tomar $\mathbb{N}=\|\mathbb{T}\|+\varepsilon)$ e também que

$$
I\left(\psi_{\operatorname{Df}(z)}\left(\hbar\left(z_{1}\right)\right)^{-1}\right)<I, \quad \forall z_{1} \in E_{I}(r) \text {. }
$$

Conclusão: para todo $r>0$, tomando $\rho=\min \left\{\rho^{\prime}, \rho^{\prime \prime}\right\}$, teremos 
$a\left(g, g^{\prime}\right)<\rho_{1} \Rightarrow\left\|z-z^{\prime}\right\|<\rho \quad A(x)<M+\mathbb{M}\{m+m+\mathbb{M}: m\}=m(\mathbb{M}+1)^{2}$ Como $\rho_{I}$ independe de $x_{2}$ temos: $d\left(g, g^{\prime}\right)<\rho_{I} \Rightarrow d\left(u(g), u\left(g^{\prime}\right)\right)=d\left(w_{g}(h), w_{g}(h)\right)=$ $=\sup _{x \in E(x)} A(x) \leqslant \eta \quad(\mathbb{M}+I)^{2}$

- que prova a continuidade de u no ponto g.

Pelo teorema 1.8, concluimos que existe $h_{f} \in H$ tal que $\left(g_{f}, h_{f}\right)$ é um atrator para $F_{f}$ e $h_{f}$ é o ponto fixo de $W_{g_{f}}$. A parte final da demonstração será decomposta em diversas etapas:

(a) Se $g \in G$ e $g$ é de classe $C^{1}$, então :

$D g \in H, \quad \Gamma_{f}(g)$ é diferenciável e

$$
D\left(\Gamma_{f}(g)\right)=w_{g}(D g)
$$

e portanto $\Gamma_{f}(g)$ é de classe $C^{I}$, pois

$$
\omega_{\mathrm{g}}\left(D_{\mathrm{g}}\right) \in \mathrm{H} \Longrightarrow \omega_{\mathrm{g}}\left(\mathrm{Dg}_{\mathrm{g}}\right) \quad \text { é contínua. }
$$

De fato: $\mathrm{Dg} \in \mathrm{H}$, pois sendo $\mathrm{g}$ lipschitziana com $L(g) \leqslant 1$, segue-se do lema 1.9 que $\left\|D_{g}\left(z_{I}\right)\right\| \leqslant I, \forall z_{I} \in E_{I}(r)$. Por outro lado, vimos na demonstração de 3.4 que 
$L\left(\psi_{f}(g)-T_{I}\right)<\left\|\mathbb{T}_{I}^{-I}\right\|^{-1}$. Então, como $\psi_{f}(g)$ é diferenciá vel, O teorema 1.10 (Iten (c)) nos permite concluir que $\psi_{f}(g)^{-1}$ é diferenciável. Sendo $\wp_{f}(g)$ também diferenciável, segue $\rightarrow$ se a diferenciabilidade de $\Gamma_{f}(g)$. Agora, $\forall x \in E_{I}(r)$ :

$$
\begin{aligned}
& D\left(\Gamma_{f}(g)\right)(x)=D \mathscr{f}_{f}(g)\left(z_{1}\right) \circ D\left[\psi_{f}(g)^{-1}\right](x)= \\
& =D f_{2}(z) \circ\left(I, D g\left(z_{1}\right)\right) \circ\left[D f_{I}(z) \circ\left(1, \operatorname{Dg}\left(z_{1}\right)\right)\right]^{-1}= \\
& =W_{g}(D g)(x)
\end{aligned}
$$

onde $z=(1, g)\left(z_{1}\right)$ e $z_{1}=\psi_{f}(g)^{-1}(x)$. Isso mostra que

$$
D\left(\Gamma_{f}(g)\right)=w_{g}(D g)
$$

(b) Se $g \in G$ é uniformemente $C^{\mathcal{I}}$, então $\Gamma_{f}(g)$ tam bém é uniformemente $c^{1}$.

Por (a), basta mostrar que $w_{g}$ (Dg) é uniformemente continua:

$$
\begin{aligned}
& \forall x, x^{\prime} \in \mathbb{E}_{1}(r):\left\|w_{g}(D g)(x)-w_{g}(D g)\left(x^{\prime}\right)\right\|= \\
& =\| D f_{2}(z) \circ\left(1, D g\left(z_{1}\right)\right) \circ\left[D f_{1}(z) \circ\left(1, D g\left(z_{1}\right)\right)\right]^{-1}- \\
& -D_{2}\left(z^{\prime}\right) \circ\left(1, D g\left(z_{1}^{\prime}\right) \circ\left[D f_{1}\left(z^{\prime}\right) \circ\left(1, D g\left(z_{1}^{p}\right)\right)\right]^{-1} \|\right.
\end{aligned}
$$


onde $\quad z=\left(z_{1}, z_{2}\right)=(I, g) \circ\left[f_{1} \circ(I, g)\right]^{\cdots 1}(x)$

$$
\text { e } \quad z^{2}=\left(z_{1}^{i}, z_{2}^{8}\right)=(1, g) \circ\left[f_{1} \circ(1, g)\right]^{-1}\left(x^{\prime}\right)
$$

Como na demonstração da continuidade da função $u$, por a plicą̧ão de 1.6 , obtemos:

$$
\begin{aligned}
& \left\|w_{g}(D g)(x) \cdots w_{g}(D g)\left(x^{\prime}\right)\right\| \leqslant\left\|D f_{2}(z)-D f_{2}\left(z^{\prime}\right)\right\|+ \\
& +\left\|D f_{2}\left(z^{8}\right)\right\|\left\{\left\|D g\left(z_{l}\right)-D g\left(z_{l}^{q}\right)\right\|+\left\|D f_{I}(z)-D f_{I}\left(z^{1}\right)\right\|+\right.
\end{aligned}
$$

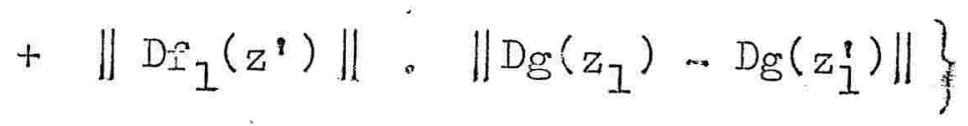

Nas :

(i) $\forall \rho>0, \quad \exists r_{L}^{\prime}>0$ tal que

$$
\left\|z-z^{3}\right\|<\eta^{\prime} \Longrightarrow\left\|D_{i}(z) \ldots \operatorname{Df}_{i}\left(z^{2}\right)\right\|<\rho
$$

pois $f \in U C^{I}$.

(ii) $\exists \mathbb{M}>0$ tal que $\left\|\mathrm{Df}_{i}\left(z^{8}\right)\right\| \leqslant \mathbb{M}, \forall z^{p} \in \mathbb{E}(r)$ $(i=1,2)$

( basta tomar $\mathbb{M}=\|T\|+\varepsilon$ )

(iii) $\forall p>0 \exists \eta \eta^{\prime}>0$ tal que 
56

$$
\left\|z_{I}-z_{I}^{\prime}\right\|<\eta_{1}^{*+} \quad \Longrightarrow\left\|D g\left(z_{I}\right)-D g\left(z_{1}^{\prime}\right)\right\|<\rho
$$

( pois Dg é uniformemente contínua).

$$
\begin{aligned}
& \text { (iv) }\left\|z-z^{\prime}\right\|=\|(I, g) \circ\left[f_{1} \circ(I, g)\right]^{-1}(x)- \\
& -(1, g) \circ\left[f_{1} \circ(1, g)\right]^{-1}\left(x^{2}\right) \| \leqslant \\
& \leqslant I(I, g) I\left\|\psi_{f}(g)^{-I}(x)-\psi_{f}(g)^{-1}\left(x^{\prime}\right)\right\| \leqslant \\
& \leqslant I(I, g) I \cdot I\left(\psi_{f}(g)^{-m}\right) \quad\left\|x-x^{2}\right\|<\left\|x-x^{\prime}\right\| \\
& \text { ( pois } I(I, g) \leqslant I \text { e } \psi^{\psi}(g)^{-I} \text { é contração ). } \\
& \text { Então: dado } \rho>0 \text {, tomando } \\
& \eta=\min \left\{\eta^{*}, \eta^{\prime \prime}\right\}, \text { teremos: } \\
& \left\|x-x^{\imath}\right\|<\eta \Longrightarrow\left\|z-z^{\prime}\right\|<\eta \Longrightarrow\left\|\omega_{g}\left(D_{g}\right)(x)-\omega_{g}\left(D_{g}\right)\left(x^{\prime}\right)\right\|< \\
& <\rho+\mathbb{M}\{\rho+\rho+\mathbb{M} \rho\}=\rho(\mathbb{M}+1)^{2}
\end{aligned}
$$

- que mostra que $\omega_{g}\left(D_{g}\right)$ é uniformemente contínua.

(c) Se $g \in U C^{\mathcal{L}}$ então a sequência $\left(D\left(\Gamma_{f}^{\mathrm{n}}(g)\right)\right)$ converge para $h_{f}$ em $H$ (isto é, uniformemente), e portanto $h_{f}$ 
é uniformemente contínua, como limite uniforme de uma sequência de funções uniformemente contínuas.

Porihamos $\Delta g=(g, D g) \quad G \quad G \times H$. Então :

$F_{f}(\Delta g)=\left(\Gamma_{f}(g), u_{g}(D g)\right)=\left(\Gamma_{f}(g), D\left(\Gamma_{f}(g)\right)=\Delta \Gamma_{f}^{\prime}(g)\right.$. Por indução, chega...se fàcilmente a $F_{f}^{n}(\Delta g)=\Delta \Gamma_{f}^{n}(g), \forall n \in \mathbb{N}$. Como, porém, $\left(g_{f}, h_{f}\right)$ é um atrator para $F_{f}$, temos $F_{f}^{n}(\Delta g) \longrightarrow\left(g_{f}, h_{f}\right)$. Logo, $\Delta \Gamma_{f}^{n}(g)=\left(\Gamma_{f}^{n}(g), D\left(\Gamma_{f}^{n}(g)\right)\right) \longrightarrow\left(g_{f}, h_{f}\right)$, donde concl $\underline{u}$ imos que $D\left(\Gamma_{f}^{n}(g)\right) \longrightarrow h_{f}$ em $H$.

(d) $h_{f}=D g_{f}$, o que termina a demonstração.

De fato: seja $g \in G$ uma função uniformemente $C^{I}$ (existe tal função; por exemplo, $g=0$ ). A sequência de funções$\left(\Gamma_{f}^{n}(g)\right)$ satisfaz as hipóteses do teorema 1.12, pois são dife renciáveis (item $(a)$ ), a sequência. $\left(\Gamma_{f}^{n}(g)(a)\right)$ converge para todo $a \cdot t E_{I}(r) \quad\left(\right.$ pois $\left.\Gamma_{f}^{n \prime}(g) \rightarrow g_{f}\right)$, e a sequência

$D\left(\Gamma_{f}^{\mathrm{n}}(\mathrm{g})\right)$ converge uniformemente em $E_{I}(r)$ (item (c)) ; logo, em qualquer bola contida em $E_{I}(r)$.

Conclusão: $D\left(\Gamma_{f}^{n}(g)\right) \longrightarrow D g_{f}$ (pontualmente) . Nas

$D\left(\Gamma_{f}^{n}(g)\right) \longrightarrow h_{f} \quad$ uniformemente, e portanto $h_{f}=D g_{f}$.

4.5 IEMA. Para todo $\tilde{r} \in \mathbb{R}$ tal que $0<\tilde{r} \leqslant r$, exis te $j \in \mathbb{N}$ tal que 
$g_{\tilde{I}}=f_{2}^{j} \circ\left(I, g_{\tilde{I}}\right) \circ\left[f_{1}^{j} \circ\left(I, g_{\tilde{I}}\right)\right]^{-I} / E_{I}(r)$ onde $\tilde{I}=f / E(\tilde{r})$ e $g_{\tilde{I}}=g_{\tilde{f}} / E(\tilde{r})$ é o ponto fixo de $\Gamma_{\tilde{I}}$.

Demonstração :

Inicialmente observemos que, como não se tem necessàriamente $\operatorname{Im} f \subset \mathbb{E}(r)$, pode não estar bem definida a composta $f \circ f \circ \ldots \circ f$. Restringindo $f$ a conjunto $A=f^{-n}(E(r))$ (ver seção 3), porém, podemos considerar a composta $(f / A) \circ(f / A) \circ \ldots \circ(f / A)$ (n vêzes), que será indicada libe ralmente por $f^{\text {h }}$. A mesma observação vale para a funcão $\psi_{f}\left(g_{f}\right)$. Seja $p=\psi_{f}\left(g_{f}\right): E_{I}(r) \rightarrow E$. Sabemos de 3.4 que $p$ é uma expansão, isto é,

$\|p(x)-p(y)\| \geqslant\left(\zeta^{-1}-\varepsilon\right)\|x-y\|, \forall x, y \in E(r)$. Afirmemos que existe $j \in \mathbb{N}$ tal que $p^{j}\left(E_{I}(\tilde{r})\right) \supset E_{1}(r)$. De fato : ponhamos $K=\zeta^{-1}-\mathcal{E}$ e consideremos uma sequên cia $\left(r_{n}\right)$ de números reais tais que

$\tilde{r}>r_{1}>r_{2}>\ldots>r_{n}>\ldots>\frac{\tilde{r}}{K}$. É claro que existe o menor $j \Leftrightarrow \mathbb{N}$ tal que $K^{j}>r_{j}>K^{j-1} \tilde{r} \geqslant r$. Por 1.ll, temos $\mathrm{p}\left(\mathrm{E}_{\perp}(\tilde{r})\right) \supset \mathrm{E}_{\perp}\left(\mathrm{Kr} r_{1}\right)$. Se $K r_{1} \geqslant r$, temos $j=1$. Se $K r_{1}<r$, temos novamente por 1.1工: $p\left(\mathbb{E}_{1}\left(K r_{1}\right)\right) \supset \mathbb{E}_{1}\left(K^{2} r_{2}\right)$ e como $E_{1}\left(K_{1}\right) \subset \mathrm{p} \cdot\left(E_{1}(\tilde{r})\right) \cap E_{1}(r)$ temos $p^{2}\left(E_{1}(\tilde{r})\right) \supset p\left(E_{1}\left(K r_{1}\right)\right) \supset E_{1}\left(K^{2} y_{2}\right)$. Se $K^{2} r_{2} \geqslant r$, temos $j=2$; caso contrário, repetimos o raciocínio, obtendo 
$p^{3}\left(E_{1}(\tilde{r})\right) \supset E_{1}\left(\pi^{3} r_{3}\right)$. Então, após j passos, chegaremosa $\quad p^{j}\left(E_{I}(\tilde{I})\right) \supset E_{1}\left(K^{j} r_{j}\right) \supset E_{I}(r)$.

Lembrando agora que $\left(I, g_{f}\right) \circ \widetilde{H}_{I}=I_{W_{I}}$ é fácil ver que $p^{j}=\pi_{I} \circ f^{j} \circ\left(I, g_{f}\right)$ donde

$\pi_{I} \circ f^{j} \circ\left(I, g_{f}\right)\left(E_{1}(\tilde{r})\right) \supset E_{I}(r)$ e portanto

$\left(I, g_{f}\right) \circ \pi_{I} \circ f^{j} \circ\left(I, g_{f}\right)\left(E_{I}(\tilde{r})\right) \supset\left(I, g_{f}\right)\left(E_{I}(r)\right)$ ou seja $f^{j}\left(\right.$ graf $\left.g_{\tilde{f}}\right) \supset \operatorname{graf} g_{f}$. Isso acarreta

(4-I) $\quad f^{j}\left(\operatorname{graf} \varepsilon_{\tilde{I}}\right) \cap E(r)=\operatorname{graf} g_{f}$.

Podemos agora provar o lema em duas etapas :

(a) $\left[f_{j}^{j} \circ\left(1, g_{\tilde{x}}\right)\right]^{-1} \quad$ está bem definida em $E_{1}(r)$ e toma valôres em $E_{1}(\tilde{r})$.

De fato: Se $x \in E_{1}(r)$, existe um único

$\left(x, g_{f}(x)\right)$ e graf $g_{f}$ - Então, por $(4-I)$, existe um único $\left(y, g_{\tilde{I}}(y)\right) \in$ graf $g_{\tilde{I}}$ tal que $f^{j}\left(y, g_{\tilde{I}}(y)\right)=\left(x, g_{\tilde{f}}(x)\right) \quad-$ (pois $f$ é bijotora). Então: $x=f_{I}^{j}\left(y, g_{\tilde{f}}(y)\right)$, o que des monstra o Item (a).

(b) $\quad g_{\tilde{I}}=f_{2}^{j} \circ\left(1, g_{\tilde{I}}\right) \circ\left[f_{1}^{j} \circ\left(1, g_{\tilde{I}}\right)\right]^{-1} / \mathrm{E}_{1}(r)$. De fato, com ass notações de (a), temos: 
$g_{f}(x)=f_{2}^{j}\left(y, g_{\tilde{f}}(y)\right)=f_{2}^{j} \circ\left(1, g_{\tilde{x}}\right)(y)=$

$=f_{2}^{j} \circ\left(I, g_{\tilde{f}}\right) \circ\left[f_{1}^{j} \circ\left(1, g_{\tilde{f}}\right)\right]^{-1}(x)$

Observação: Será útil notar que

$f_{I}^{j} \circ\left(I, g_{\tilde{I}}\right)=\left[\underline{f}_{I} \circ\left(I, g_{f}\right)\right]^{j-1} \circ f_{I} \circ\left(I, g_{\tilde{I}}\right)$

( pois

$\pi_{I} \circ f \circ\left(1, g_{f}\right) \circ \pi_{I} \circ f \circ\left(1, g_{f}\right) \circ \ldots \circ \pi_{I} \circ f \circ\left(1, g_{\tilde{f}}\right)=$

$\left.=\pi_{I} \circ f^{j} \circ\left(I, g_{\tilde{I}}\right)\right)$ e que portanto $f_{I}^{j} \circ\left(I, g_{\tilde{I}}\right)$ é um difeomor fismo quando $f \in U C^{I}$.

4.6 IENA - Seja $\left(g_{n}\right)$ uma sequência de funções de $E(r)$ em $F, E \in F$ espaços normados. Então, $g_{n}^{*} \stackrel{C^{p}}{\longrightarrow} g^{*}$ se, e sòmente se, $g_{n} \stackrel{c^{p+1}}{\longrightarrow} g \quad(p \geqslant 1)$.

Demonstração.

Suponhamos em primeiro lugar que $g_{n} \stackrel{c^{p+1}}{\longrightarrow} g$. Então, $D g_{n} \stackrel{C^{p}}{\longrightarrow} D g \quad$ e $\quad D^{2} g_{n} \stackrel{C^{p-1}}{\longrightarrow} D^{2} g$, o que acarreta, devido a

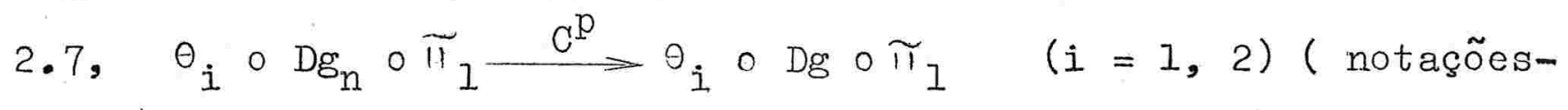
de 2.14) e

$\theta_{1} \circ v \circ\left(D^{2} g_{n} \circ \pi_{1}, \pi_{2}\right) \stackrel{C^{p-1}}{\longrightarrow} \theta_{1} \circ v \circ\left(D^{2} g \circ \pi_{1}, \pi_{2}\right)$. Pela proposição 2.14, podemos concluir que $D\left(g_{n}^{*}\right) \stackrel{\mathrm{C}^{\mathrm{p}-1}}{\longrightarrow} D\left(\mathrm{~g}^{*}\right)$, donde $\quad g_{n}^{*} \stackrel{C^{p}}{\longrightarrow} g^{*}$. 
Reciprocamente, so $g_{\mathrm{n}}^{*} \stackrel{\mathrm{C}^{\mathrm{p}}}{\longrightarrow} \mathrm{g}^{*}$, então $D\left(g_{n}^{*}\right) \stackrel{c^{p-1}}{\longrightarrow} D\left(g^{*}\right)$, e novamente pela proposição 2.14 , temos: (i) $\theta_{1} \circ \operatorname{Dg}_{\mathrm{n}} \circ \pi_{1} \stackrel{\mathrm{C}^{\mathrm{p}-1}}{\longrightarrow} \theta_{1} \circ \mathrm{Dg} \circ \pi_{1}, \mathrm{e}$ (ii) $\theta_{2} \circ \mathrm{Dg}_{\mathrm{n}} \circ \widetilde{\pi}_{1}+\theta_{1} \circ \mathrm{v}\left(\mathrm{D}^{2} \mathrm{~g}_{\mathrm{n}} \circ \pi_{1}, \pi_{2}\right) \stackrel{\mathrm{C}^{\mathrm{p}-1}}{\longrightarrow} \theta_{2} \circ \mathrm{Dg} \circ \pi_{1}+$ $+\theta_{1} \circ \mathrm{v} \circ\left(D^{2} g \circ \widetilde{\pi}_{1}, \pi_{2}\right)$.

A proposição 2.7 permite concluir então de (i) que $D g_{n} \stackrel{{ }^{p}-1}{\longrightarrow} D g$ e portanto de (ii) que $D^{2} g_{n} \stackrel{C^{p-1}}{\longrightarrow} D^{2} g$, donde $g_{n} \stackrel{\mathrm{C}^{\mathrm{p}+1}}{\longrightarrow} \mathrm{g}$. De fato: mostremos que

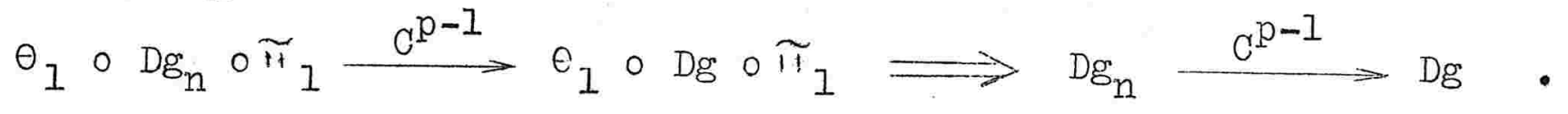
Para isso, definamos as aplicações $\eta_{0}, \eta_{1}, \ldots, \eta_{p-1}$ do se guinte modo: $\eta_{0}=\theta_{1}: I\left(E_{1}, E_{2}\right) \longrightarrow I\left(E_{1} \times I_{1}, E_{2}\right)$ e para $i=1,2, \ldots, p-1$,

$\eta_{i}: I\left(E_{1}, I\left(E_{1}, \ldots, I\left(E_{1}, E_{2}\right)\right)\right) \rightarrow I\left(E_{1} \times E_{1}, I\left(E_{1} \times E_{1}, \ldots\right.\right.$ $\left.\left.\ldots, I\left(E_{1} \times E_{1}, E_{2}\right)\right)\right)$ é tal que $\eta_{i}(u)=\eta_{i-1} \circ u \cdot \pi_{1}$. É fácil ver que se trata de aplicações lineares contínuas. Se pusermos $d=\theta_{1} \circ\left(D_{n}-D g\right) \circ \pi_{1}$, um cálculo pacientepermite chegar, com o auxílio de 2.7 , a

$D_{\alpha^{\prime}}^{j}=\eta_{j} \circ\left(D^{j+1} g_{n}-D^{j+1} g\right) \circ \pi_{1}, j=0,1, \ldots, p-1$

Então : para $x, y \in E_{1}(r)$, temos :

$\left\|D_{\alpha}^{j} \quad(x, y)\right\|=\left\|\eta_{j} \circ\left(D^{j+1} g_{n}-D^{j+1} g\right)(x)\right\|=$ 
62

$$
\begin{aligned}
& =\left\|\eta_{j-1} \circ\left[D^{j+1} g_{n}(x)-D^{j+1} g(x)\right] \circ \tilde{H}_{1}\right\|= \\
& =\left\|\sup _{1}\right\| \leqslant 1 \quad\left\|\eta_{j-1} \circ\left[D^{j+1} g_{n}(x)-D^{j+1} g(x)\right] \quad\left(a_{1}\right)\right\| \\
& =\sup _{\left\|a_{1}\right\| \leqslant 1}\left\|\eta_{j-2} \circ\left[D^{j+1} g_{n}(x)-D^{j+1} g(x)\right] a_{1} \circ \pi_{1}\right\|=
\end{aligned}
$$

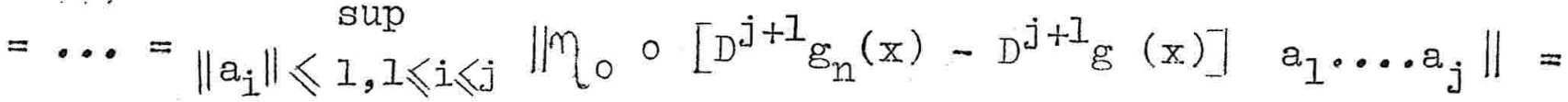

$$
\begin{aligned}
& =\sup _{\left\|a_{i}\right\| \leqslant 1,1<i \leqslant j}\left\|\left[D^{j+1} g_{n}(x)-D^{j+1} g(x)\right] a_{1} \ldots a_{j} \circ \pi_{1}\right\|= \\
& \sup _{\left\|a_{i}\right\| \leqslant 1,1 \leqslant i \leqslant j+1}\left\|\left[D^{j+1} g_{n}(x)-D^{j+1} g(x)\right] \quad a_{1} \ldots a_{j+1}\right\|= \\
& =\left\|D^{j+1} g_{n}(x) \quad \cdots D^{j+1} g(x)\right\| \text {. Mas, devido a (i), sabe - }
\end{aligned}
$$

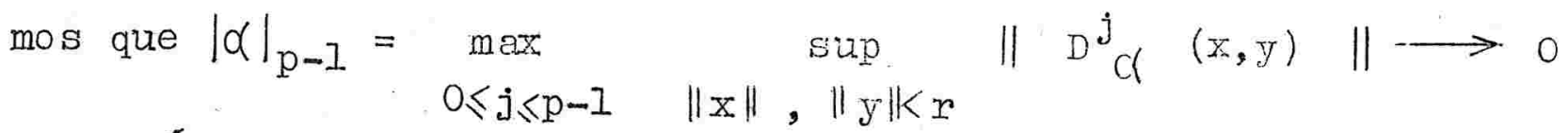

Ago ra, de (ii), concluímos que

$\theta_{1} \circ \mathrm{v} \circ\left(D^{2} \mathrm{~g}_{\mathrm{n}} \circ \pi_{1}, \pi_{2}\right) \stackrel{C^{p-1}}{\longrightarrow} \theta_{1} \circ \mathrm{v} \circ\left(D^{2} \mathrm{~g} \circ \pi_{1}, \pi_{2}\right)$. Mo stremos para $p=1$ e $p=2$ que daí segue $D^{2} g_{n} \stackrel{C^{p-1}}{\longrightarrow} D^{2} g \quad(a$ demonstração geral se faz por indução ). Sejam

$K_{n}=\theta_{1} \circ v \circ\left(D^{2} g_{n} \circ \pi_{1}, \pi_{2}\right)$ e $K=\theta_{1} \circ v \circ\left(D^{2} g \circ \pi_{1}, \pi_{2}\right)$

$\mathrm{p}=1 \quad \forall \varepsilon>0, \exists \mathrm{n}_{0} \in \mathbb{N}$ tal que

$$
\mathrm{n} \geqslant \mathrm{n}_{0} \Rightarrow\|\mathrm{x}\|,\|y\|<r \quad\left\|\mathrm{sup}_{n}(\mathrm{x}, \mathrm{y})-\mathrm{K}(\mathrm{x}, \mathrm{y})\right\|<\frac{\varepsilon r}{2} \Longrightarrow
$$


$\Longrightarrow\|x\|, \sup _{\| y}\|<r\| D^{2} g_{n}(x)$ y $\circ \pi_{I}-D^{2} g(x)$ y $\circ \pi_{I} \|<\frac{\varepsilon r}{2}$

$\Rightarrow\|x\|, \quad \sup _{\| y} \sup _{\|a\|}\left\|D^{2} g_{n}(x) \cdot y \cdot a-D^{2} g(x) \cdot y \cdot a\right\|<\frac{\varepsilon r}{2}$

$\Rightarrow \sup _{x \|<r} \sup _{\|a\| \leqslant 1} \frac{r}{2}\left\|D^{2} g_{n}(x) a-D^{2} g(x) a\right\|<\frac{\varepsilon r}{2}$

$\Rightarrow\|x\|<r \quad\left\|D^{2} g_{n}(x)-D^{2} g(x)\right\|<\varepsilon \Longrightarrow\left|D^{2} g_{n}-D^{2} g\right|_{0}<\varepsilon$

$p=2 \quad$ Se $(x, y),(a, b) \in E_{1} \times E_{1}$, temos por 2.10: $\operatorname{DIS}(x, y)(a, b)=\Theta_{1} \circ \operatorname{DV}\left(D^{2} g(x), y\right) \circ\left(D^{3} g(x) \circ \pi_{1}, \pi_{2}\right)(a, b)=$ $=\operatorname{Dv}\left(D^{2} g(x), y\right)\left(D^{3} g(x) a, b\right) \circ \pi_{I}=$

$=\left(D^{2} g(x) b+D^{3} g(x) \cdot a \cdot y\right) \circ \pi_{I}=D^{2} g(x) b \circ \pi_{I}+D^{3} g(x) \cdot a \cdot y \circ \pi^{*} I$ Então :

$D K(x, y)=\theta_{1} \circ D^{2} g(x) \circ \pi_{2}+\theta_{1} \circ D^{3} g(x)$ y $\circ \pi_{1}$ donde, indicando por $\rho$ a composição à esquerda com $\theta_{I}$, obtemos: $D K=\rho \circ \theta_{2} \circ D^{2} g \circ \pi_{1}+\rho \circ \theta_{1} \circ v \circ\left(D^{3} \mathrm{~g}_{0} \circ \pi_{1}, \pi_{2}\right)($ análogo para $D K r)$. Agora, se $K_{n} \stackrel{C^{I}}{\longrightarrow} K$, então $D K n \stackrel{C^{O}}{\longrightarrow} D K$. Mas $\mathrm{K}_{\mathrm{n}} \stackrel{\mathrm{C}^{\mathrm{I}}}{\longrightarrow} \mathrm{K} \Rightarrow \mathrm{K}_{\mathrm{n}} \stackrel{\mathrm{C}^{0}}{\longrightarrow} \mathrm{K} \Rightarrow \mathrm{D}^{2} \mathrm{~g}_{\mathrm{n}} \stackrel{\mathrm{C}^{0}}{\longrightarrow} \mathrm{D}^{2} \mathrm{~g} \quad$ ( $\mathrm{já}$ demonstrado). ConcluÍmos por 2.7 que

$\rho \circ \theta_{2} \circ D^{2} g_{n} \circ \pi_{1} \stackrel{C^{\circ}}{\longrightarrow} \rho \circ \theta_{2} \circ D^{2} g \circ \pi_{1} \circ$ que portanto $\rho \circ \theta_{1} \circ v \circ\left(D^{3} g_{n} \circ \pi_{1}, \pi_{2}\right) \stackrel{C^{\circ}}{\longrightarrow} \rho \circ \theta_{1} \circ v \circ\left(D^{3} g \circ \pi_{1}, \pi_{2}\right)$ De um modo análogo ao que se fêz no caso $p=1$, concluímos que $D^{3} g_{n} \stackrel{C^{0}}{\longrightarrow} D^{3} g$ ou seja $\quad D^{2} g_{n} \stackrel{C^{I}}{\longrightarrow} D^{2} g$. 
4.7 PROPOSIÇOAO. Seja $f: E(r) \rightarrow E$ como no lema 4.1 e suponhamos que $f \in U C^{k}(1 \leqslant k<\infty)$. Então existe $\hat{r} \leqslant r$ tal que $g_{\hat{f}}=g_{f} / E(\hat{r})$ é uniformemente $C^{k}$.

Demonstração .

Façamos indução sôḅre $k$. O caso $k=1$ foi demonstrado em 4.4 , com $\hat{r}=r$. Suponhamos então válida a proposi ção para funções uniformemente $\mathrm{C}^{\mathrm{k}-1}$, com $k \geqslant 2$, e provemos que vale para funções uniformemente $C^{k}$. Seja $\mathbb{M}$ tal que $|f|_{k} \leqslant \mathbb{M}$ e sejam $\stackrel{*}{\xi}$ e $\tilde{r}$ tais que

$\varepsilon<\tilde{\varepsilon}<\min \left\{\frac{1-\zeta}{1+\zeta},\left\|T^{-1}\right\|^{-1}\right\}, 0<\tilde{r} \leqslant r$, e $\mathbb{M} \tilde{r}<\tilde{\varepsilon}-\varepsilon$ Indicaremos $\tilde{\tilde{f}}=f / E(\tilde{r})$.

Consideremos o operador TX T:EXE $\longrightarrow \mathrm{EXE}$ tal que $T x T(x, y)=(T x, T y)$. É trivial verificar que $T x T$ é um operador hiperbólico (seu espectro está contido no espectro de $T$, uma vez que $T-\lambda I$ é injetor (sobrejetor) se, e sòmente se, $T X T-\hat{\lambda}$ ( $I x I)$ é injetor (resp., sobrejetor)) e que a de composição canônica de $E \times E$ a êle associada é

$\left(E_{1} \times E_{1}\right) \oplus\left(E_{2} \times E_{2}\right)$. Consequentemente, se $u=\left(u_{1}, u_{2}\right)$ e $v=\left(v_{1}, v_{2}\right)$ são dois elementos de $E, \operatorname{com} u_{i}, v_{i} \in E_{i}$ $(i=1,2)$, o elemento $(u, v)$ de $\mathrm{E} \times \mathrm{E}$ passará a ser indicado por $\left(\left(u_{1}, v_{1}\right),\left(u_{2}, v_{2}\right)\right)$, isto é, as suas coordenadas serão $\left(u_{1}, v_{1}\right)$ e $\left(u_{2}, v_{2}\right)$. Também é fácil ver que $(\mathrm{T} \times \mathrm{T})_{i}=\mathrm{T}_{i} \times \mathrm{T}_{i},\left\|\mathrm{~T}_{i} \times \mathrm{T}_{i}\right\|=\left\|\mathrm{T}_{i}\right\|$, 
$\left(\mathrm{T}_{i} \times \mathrm{T}_{i}\right)^{-1}=\mathrm{T}_{i}^{-1} \times \mathrm{T}_{i}^{-1}$ e $\left\|\left(\mathrm{T}_{i} \times \mathrm{T}_{i}\right)^{-1}\right\|=\left\|\mathrm{T}_{i}^{-1}\right\|$

$(i=1,2)$. Segue-se que $T x T$ tem assimetria $\zeta$.

Lembremos agora que $\tilde{I}^{*}:(E \times E)(\tilde{r}) \longrightarrow E X E$ é de finida por $\tilde{I}^{*}(x, y)=(\tilde{I}(x), D \tilde{I}(x) \cdot y)(\operatorname{ver} 2.13)$. Se $\tilde{f}_{1}^{*}$ e $\tilde{f}_{2}^{*}$ indicam suas componentes segundo a decomposição canônica de EXE , temos : $\tilde{\mathrm{f}}_{i}^{*}=\left(\tilde{\mathrm{f}}_{i}\right)^{*} \quad(i=1,2)$. De fato: se $u=\left(u_{1}, u_{2}\right), v=\left(v_{1}, v_{2}\right) \in E(\tilde{r}), 0$ elemento $\tilde{I}^{*}(u, v)=(\tilde{I}(u), D \tilde{f}(u) \cdot v) \in E X E$ se escreve,de acôrdo com o que observamos anteriormente,

$\left(\left(\tilde{f}_{1}(u), D \tilde{f}_{I}(u) v\right),\left(\tilde{f}_{2}(u), D \tilde{f}_{2}(u) v\right)\right)$ ou seja $\left(\left(\tilde{f}_{1}\right)^{*}(u, v),\left(\tilde{I}_{2}\right)^{*}(u, v)\right)$.

Sabemos de 2.13 que $\tilde{I}^{*}$ é uniformemente $C^{k-I}$. Mostre mos então que $I\left(\tilde{x}^{*}-T \times T\right)<\tilde{\varepsilon}$, e assim estará satisfeita a hipótese de inducão: $\forall x, y, x^{\prime}, y^{\prime} \in E(\tilde{r})$;

$$
\begin{aligned}
& \left\|\left(x^{*}-T X T\right)(x, y)-\left(\tilde{f}^{*}-T x T\right)\left(x^{\prime}, y^{\prime}\right)\right\|= \\
& =\left\|\tilde{f}^{*}(x, y)-T X T(x, y)-\tilde{x} *\left(x^{9}, y^{\prime}\right)+T x T\left(x^{\prime}, y^{\prime}\right)\right\|= \\
& =\|\left(\tilde{f}_{1}^{*}(x, y), \tilde{f}_{2}{ }^{*}(x, y)\right)-\left(\mathbb{T}_{1} \times T_{1}\left(x_{1}, y_{1}\right), T_{2} \times T_{2}\left(x_{2}, y_{2}\right)\right)- \\
& \text { - }\left(\tilde{f}_{1}^{*}\left(x^{\prime}, y^{\prime}\right), \tilde{f}_{2}^{*}\left(x^{\prime}, y^{p}\right)\right)+\left(T_{1} \times T_{1}\left(x_{1}^{p}, y_{1}^{p}\right), T_{2} \times T_{2}\left(x_{2}^{p}, y_{2}^{p}\right)\right) \| \\
& =\max \left\{\left\|\tilde{f}_{1}^{*}(x, y)-T_{1} \times T_{1}\left(x_{1}, y_{1}\right)-\tilde{x}_{1}^{*}\left(x^{\prime}, y^{\prime}\right)+T_{1} \times T_{1}\left(x_{1}^{\prime}, y_{1}^{\prime}\right)\right\|\right. \text {, } \\
& \left.\left\|\tilde{f}_{2}^{*}(x, y)-T_{2} \times T_{2}\left(x_{2}, y_{2}\right)-\tilde{f}_{2}^{*}\left(x^{*}, y^{\prime}\right)+T_{2} x \mathbb{T}_{2}\left(x_{2}^{\prime}, y_{2}^{\prime}\right)\right\|\right\}
\end{aligned}
$$


Mas, para $i=1,2$, temos :

$$
\left\|\tilde{f}_{i}^{*}(x, y)-T_{i} \times T_{i}\left(x_{i}, y_{i}\right)-\tilde{f}_{i}^{*}\left(x^{\prime}, y^{\prime}\right)+T_{i} \times T_{i}\left(x_{i}^{\prime}, y_{i}^{\prime}\right)\right\|=
$$

$\|\left(\tilde{f}_{i}(x), D \tilde{f}_{i}(x) y\right)-\left(T_{i} x_{i}, T_{i} y_{i}\right)-\left(\tilde{f}_{i}\left(x^{\prime}\right), D \tilde{f}_{i}\left(x^{\prime}\right) y^{\prime}\right)+$

$$
+\left(\mathrm{T}_{i} \mathrm{x}_{i}^{\prime}, \mathrm{T}_{i} \mathrm{y}_{i}^{\prime}\right) \|=
$$

$=\left\|\left(\tilde{f}_{i}(x)-T_{i} x_{i}-\tilde{f}_{i}\left(x^{\prime}\right)+T_{i} x_{i}^{p}, D \tilde{f}_{i}(x) y-T_{i} y_{i}-D \tilde{f}_{i}\left(x^{t}\right) y^{\prime}+T_{i} y_{i}^{\prime}\right)\right\|=$

$=\left\|\widetilde{\pi}_{i}\left[(\tilde{f}-T)(x)-(\tilde{f}-T)\left(x^{\prime}\right)\right], \widetilde{\pi}_{i}\left[(D \tilde{f}(x)-T)(y)-\left(D \tilde{f}\left(x^{\prime}\right)-T\right)\left(y^{\prime}\right)\right]\right\|=$

$=\max \left\{\left\|\tilde{r}_{i}\left[(\tilde{f}-T)(x)-(\tilde{I}-T)\left(x^{\prime}\right)\right]\right\|, \| \pi_{i}\left[(D \tilde{f}(x)-T)(y)-\left(D \tilde{f}\left(x^{\prime}\right)-T\right)\left(y^{1}\right)\right].\right\} \leqslant$

$\leqslant \max \left\{\left\|(\tilde{f}-T)(x)-(\tilde{f}-T)\left(x^{\prime}\right)\right\|,\left\|(D \tilde{f}(x)-I)(y)-\left(D \tilde{f}\left(x^{\prime}\right)-T\right)\left(y^{\prime}\right)\right\| \leqslant\right.$

$\leqslant \max \left\{L(\tilde{f}-T)\left\|x-x^{\prime}\right\|,\left\|D \tilde{f}(x)-D \tilde{f}\left(x^{\prime}\right)\right\| \cdot\|y\|+\right.$

$$
\left.+\left\|\tilde{D} \tilde{Y}\left(x^{\prime}\right)-T\right\| \cdot\left\|y-y^{\prime}\right\|\right\}
$$

$<\max \left\{\varepsilon\left\|x-x^{\prime}\right\|,\left\|D \tilde{f}(x)-D \tilde{f}\left(x^{\prime}\right)\right\| \tilde{x}+\varepsilon\left\|y-y^{\prime}\right\|\right\}$

( pois conforme foi visto na demonstração de 4.4 , temos $\|\mathrm{D} \tilde{\mathrm{f}}(\mathrm{z})-\mathrm{T}\|<\varepsilon, \quad \forall z \in \mathbb{E}(\tilde{\mathrm{r}}))$ : 
Ora, sendo $\tilde{f}$ de classe $C^{k}, k \geqslant 2$, e $|\tilde{f}|_{k} \leqslant M$, segue-se do Teorema do Valor Médio que Df̃ é lipschitziana e $L(D \tilde{f}) \leqslant M \quad($ V.1.9).

Daí,

$\left\|D \tilde{f}(x)-D \tilde{f}\left(x^{\prime}\right)\right\| \tilde{r}+\varepsilon\left\|y-y^{\prime}\right\| \leqslant M \tilde{r}\left\|x-x^{\prime}\right\|+\varepsilon\left\|y-y^{\prime}\right\|^{\prime}$

$\leqslant(M \tilde{r}+\varepsilon) \max \left\{\left\|x-x^{\prime}\right\|,\left\|y-y^{\prime}\right\|\right\}<\tilde{\varepsilon}\left\|(x, y)-\left(x^{\prime}, y^{\prime}\right)\right\|$

Concluimos que, para $i=1,2$, temos:

$$
\begin{aligned}
\| \tilde{f}_{i}^{*}(x, y)-T_{i} \times T_{i}\left(x_{i}, y_{i}\right)-\tilde{f}_{i}^{*}\left(x^{\prime}, y^{\prime}\right) & +T_{i} x T_{i}\left(x_{i}^{\prime}, y_{i}^{f}\right) \|< \\
& <\tilde{\varepsilon}\left\|(x, y) \ldots\left(x^{f}, y^{1}\right)\right\|
\end{aligned}
$$

donde

$$
\left\|\left(\tilde{f}^{*}-T \times T\right)(x, y)-\left(\tilde{f}^{*}-T \times T\right)\left(x^{\prime}, y^{\prime}\right)\right\|<\tilde{\varepsilon}\left\|(x, y)-\left(x^{\prime}, y^{\prime}\right)\right\|
$$

e portanto $\quad I\left(\tilde{I}^{* *}-T \times T\right)<\tilde{E}$.

Pela hipótese de indução, a função

$$
\mathrm{g}_{\tilde{f}^{*}}:\left(\mathbb{E}_{1} \times E_{1}\right)(\tilde{r}) \longrightarrow\left(E_{2} \times E_{2}\right)(\tilde{r}),
$$

ponto fixo da transformação-gráfico

$$
\Gamma_{\tilde{f}}:: \tilde{G}_{2} \rightarrow \tilde{G}_{2}=\left\{g \in M\left(\left(E_{1} \times E_{1}\right) \overline{(\tilde{r}),\left(E_{2} \times E_{2}\right.}\right)(\tilde{r}): L(g) \leqslant 1\right\},
$$


é uniformemente $C^{\mathrm{k}-1}$ em uma bola $E(\hat{r})$, com $\hat{r} \leqslant \tilde{r}$. A seguir, estabeleceremos uma relação entre $g_{\hat{f}^{*}}$ e $g_{\hat{f}}$, que são, como é fácil ver, as restrições de $g_{f^{*}}$ e $g_{f}$ às bolas $\left(E_{I} \times E_{I}\right)(\hat{r})$ e $\mathrm{E}(\hat{r})$.

$$
\begin{aligned}
& \text { Ponhamos } \\
& \hat{G}=\left\{g \in \mathbb{M}\left(E_{1}(\hat{r}), \overline{E_{2}(\hat{r})}\right): I(g) \leqslant I\right\} \\
& \hat{H}=\left\{h \in C^{\circ}\left(E_{1}(\hat{r}), L\left(E_{1}, E_{2}\right):\|h(x)\| \leqslant I, \quad \forall x \in E_{1}(\hat{r})\right\}\right.
\end{aligned}
$$

Anàlogamente ao que se fêz na demonstração de 4.4 , pode mos definir a $f$ unção

$$
F_{\hat{E}}: \hat{G} \times \hat{H} \longrightarrow \hat{G} \times \hat{H}
$$

tal que

$$
F_{\hat{f}}(g, h)=\left(\Gamma_{\hat{f}}(g), \omega_{g}(h)\right)
$$

onde

$$
w_{g}(h)(x)=D \hat{f}_{2}(z) \circ\left(I, h\left(z_{1}\right)\right) \circ\left[D \hat{f}_{1}(z) \circ\left(I, h\left(z_{1}\right)\right)\right]^{-1}
$$

para todo $h \in \hat{H}, \quad x \in E_{1}(\hat{r})$, sendo $z=(I, g)\left(z_{l}\right)$ e

$$
z_{1}=\left[\hat{f}_{1} \circ(1, g)\right]^{-1}(x) \text {. Sabemos que } F_{\hat{f}} \text { contrai as fibras e }
$$

tem um atrator $\Delta g_{\hat{f}}=\left(g_{\hat{f}}, D_{\hat{f}}\right) \in \hat{G} \times \hat{H}$. Afirmamos agora que se $g \in \hat{G}$ é de classe $\mathrm{C}^{\mathcal{I}}$ e se $\psi_{\hat{f}^{*}}\left(\mathrm{~g}^{*}\right)={\hat{f^{*}}}_{1} \circ\left(I_{s} \xi^{*}\right)$ é inversível e nêsse caso existe $\Gamma_{\hat{f}^{*}}\left(g^{*}\right)$ - então vale a seguinte " comutatividade " 
(4.II) $\quad \Gamma_{\hat{f}^{*}}(g *)=\Gamma_{\hat{f}}(g) *$

que pode ser visualisada através do diagrama

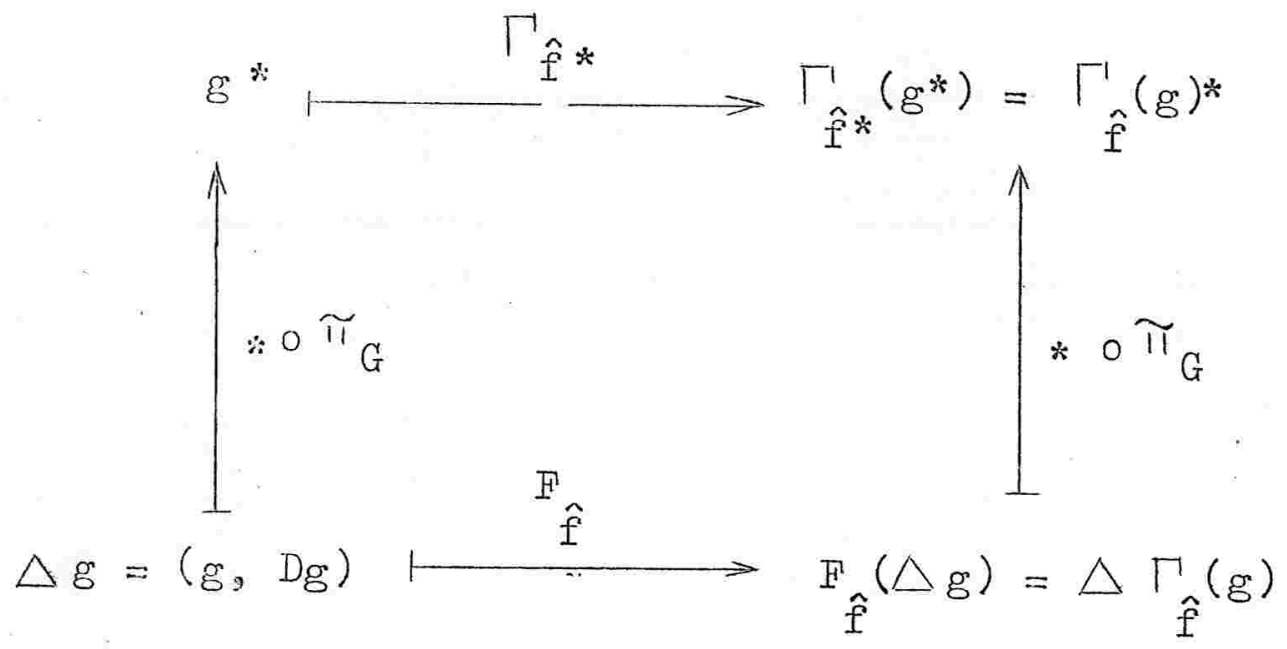

Para a demonstração dêsse fato, vide lema 4.8 .

Seja agora $g=0: \mathrm{E}_{1}(\hat{r}) \longrightarrow \mathrm{E}_{2}(\hat{r})$. $\hat{\mathrm{E}}$ claro que

$g^{*} \ominus \hat{G}_{2}$ e portanto $\psi_{\hat{f}}{ }^{*}\left(g^{*}\right)$ é inversivel, valendo (4.II).

o fato de $\hat{G}_{2}$ ser invariante por $\Gamma_{\hat{f}^{*}}$ nos permite então afirmar que, para todi $n \in \mathbb{N}$,

(4-III) $\quad \Gamma_{\hat{f} *}^{n}(g *)=\Gamma_{\hat{f}}^{n}(g) *$

( isso se demonstra fàcilmente por indução.).

Estamos então nas condições do lema 4.6, uma vez que, 
como foi visto na dẹmonstração de 4.4 , temos:

(i) para to do $n \in \mathbb{N}, \quad \Gamma_{\hat{f}}^{n}(g) \in U C^{1}$

(ii) $\Delta \Gamma_{\hat{f}}^{n}(g)=F_{\hat{f}}^{n}(\Delta g) \rightarrow\left(g_{\hat{f}}, D g_{\hat{f}}\right)=\Delta g_{\hat{f}}$ e portanto

$$
\Gamma_{\hat{f}}^{n}(g) \stackrel{c^{I}}{\longrightarrow} g_{\hat{f}}
$$

Conclusão : $\Gamma_{\hat{f}}^{n}(g)^{*} \stackrel{C^{0}}{=} g_{\hat{f}}^{*} \quad$ e portanto a convergência se dá na topologia de $\hat{G}_{2}$. Por outro lado, sabemos que

$\Gamma_{\hat{f}^{*}}^{n}\left(g^{*}\right) \longrightarrow g_{\hat{f}}^{*}$. Segue-se então de $(4-I I I)$ que

$$
g_{\hat{f}}^{*}=g_{\hat{f}}^{*}
$$

e que portanto $g_{\hat{f}}^{*} \in \mathrm{UC}^{\mathrm{k}-1}$, donde, pela proposição 2.13 , $g_{\hat{f}} \in U C^{k}$

OBSERVACXO : Do Iema 4.5 e da observecão feita após a sua demonstração, concluímos que $g_{f}$ é de classe $c^{k}$ em tôda a bola $E_{I}(r)$ pois $f_{I}^{j} \circ\left(I, g_{\hat{f}}\right)$ sendo um difeomorfismo o de clas se $c^{k}$,sua inversa também será de classe $C^{k}$ (Teorema da função inversa, ver [8] ). 
4.8 Lenia. Se $g \in \hat{G}$ é de classe $C^{\mathcal{I}}$ e se

$\psi_{\hat{f}^{*}}\left(g^{*}\right)=\hat{f}_{1}^{*} \circ\left(1, g^{*}\right)$ é inversível, então

$$
\Gamma_{\hat{f} *}(g *)=\Gamma_{\hat{f}}(g)^{*}
$$

Demonstração :

Se $\psi_{\hat{f}^{*}}\left(g^{*}\right)$ é inversĩvel, temos

$(4-I V) \quad \Gamma_{\hat{f}^{*}}\left(g^{*}\right)=\hat{f}_{2}^{*} \circ\left(I, g^{*}\right) \circ\left[\hat{f}_{I}^{*} \circ\left(I, g^{*}\right)\right]^{-1} /\left(E_{I} \times E_{I}\right)(\hat{r})$

Alếm disso, se $\mathrm{x}, \mathrm{y} \in \mathbb{E}_{\mathcal{I}} \times \mathbb{E}_{\mathcal{I}}(\hat{r})$, então

$$
\begin{aligned}
& \Gamma_{\hat{f}}(g)^{*}(x, y)=\left(\Gamma_{\hat{f}}^{\prime}(g)(x), D \Gamma_{\hat{f}}(g)(x) y\right)= \\
& =\left(\hat{f}_{2} \circ(I, g) \circ\left[\hat{f}_{I} \circ(I, g)\right]^{-I}(x), \omega_{g}(D g)(x) y\right)= \\
& =\left(\hat{f}_{2}(z), D \hat{f}_{2}(z) \circ\left(I, D g\left(z_{1}\right)\right) \circ\left[D \hat{f}_{1}(z) \circ\left(I, D g\left(z_{1}\right)\right)\right]^{-1} y\right)
\end{aligned}
$$

Seja $w=\left(1, \operatorname{Dg}\left(z_{1}\right)\right) \circ\left[D \hat{f}_{1}(z) \circ\left(1, \operatorname{Dg}\left(z_{1}\right)\right)\right]^{-1} \cdot y \in E(\hat{r})$ Então :

$(4-V) \quad \Gamma_{\hat{f}}(g)^{*}(x, y)=\left(\hat{f}_{2}(z), D \hat{f}_{2}(z) w\right)=\hat{f}_{2}^{*}(z, w)$ 
Mas :

$$
(z, w)=\left((1, g) z_{1},\left(I, D g\left(z_{1}\right) u\right)\right.
$$

onde

$$
u=\left[D \hat{f}_{1}(z) \circ\left(I, D g\left(z_{I}\right)\right)\right]^{-1} \cdot y \in E_{1}(\hat{r})
$$

Io go,

$$
(z, w)=\left(\left(z_{I}, u\right),\left(g\left(z_{I}\right), D g\left(z_{I}\right) u\right)\right)=\left(I, g^{*}\right)\left(z_{I}, u\right)
$$

Sukstituindo em (4-V), obtemos:

$(4-V I) \quad \Gamma_{\hat{f}}(g)^{*}(x, y)=\hat{f}_{2}^{*} \circ\left(I, g^{*}\right)\left(z_{1}, u\right)$

Por outro lado,

$\hat{f}_{1}^{*} \circ\left(1, g^{*}\right)\left(z_{1}, u\right)=\hat{f}_{I}^{*}\left(\left(z_{1}, u\right),\left(g\left(z_{1}\right), \operatorname{Dg}\left(z_{1}\right) u\right)\right)=$

$$
=\hat{f}_{I}^{*}(z, w)=\left(\hat{f}_{I}(z), D \hat{f}_{I}(z) w\right)=(x, y)
$$

Seguemse dal que

$$
\left(z_{1}, u\right)=\left[\hat{f}_{1}^{*} \circ\left(1, g^{*}\right)\right]^{-1}(x, y)
$$

Finalmente, por substituição em (4-VI), obtemos :

$$
\begin{array}{r}
\Gamma_{\hat{f}}(g)^{*}(x, y)=\hat{f}_{2}^{*} \circ\left(I, g^{*}\right) \circ\left[\hat{f}_{1}^{*} \circ\left(I, g^{*}\right)\right]^{-1}(x, y)= \\
=\Gamma_{\hat{f}^{*}}\left(g^{*}\right)(x, y)
\end{array}
$$


Indiquemos por $\mathbb{N}_{\varepsilon}^{k}(T)$ o conjunto

$$
\left\{f \in U C^{k}(E(r), E): L(f-T)<\varepsilon \quad \text { e } f(0)=0\right\}
$$

com a topologia induzida por $\mathrm{C}^{k}(\mathrm{E}(\mathrm{r}), \mathrm{E})$ onde $\mathrm{T}: \mathrm{E} \longrightarrow \mathrm{E} \in \mathrm{fum}$ operadr hiperbólico e

$$
\varepsilon<\min \left\{\frac{1-6}{1+z},\left\|T^{-1}\right\|^{-1}\right\}
$$

( lembremos que por 2.2, quando U $\subset \mathbb{E}$ é um aberto convexo e limi tado, tem-se $U C^{k}(U, F) \subset C^{k}(U, F)$ ).

4.9 PROPOSIÇAO A funç̃̃o

$$
\begin{aligned}
& \mathbb{N}_{\varepsilon}^{k}(T) \longrightarrow C^{k}\left(E_{1}(r), E_{2}(r)\right) \\
& f \longmapsto g_{f}
\end{aligned}
$$

é contínua.

Demonstração :

Façamos indução sôbre $k$. Seja inicialmente $k=0$. Se $f, f^{\prime} \in \mathbb{N}_{\varepsilon}^{O}(T)$, temos para cada $g \in G$, aplicando 1.6 :

$d\left(\Gamma_{f}(g), \Gamma_{f^{\prime}}(g)\right)=d\left(\varphi_{f^{\prime}}(g) \circ \psi_{f}(g)^{-1}, \varphi_{f^{\prime}}(g) \circ \psi_{f^{\prime}}(g)^{-1}\right) \leqslant$

$\leqslant d\left(\varphi_{f}(g), \varphi_{f^{\prime}}(g)\right)+L\left(\varphi_{f^{\prime}}(g)\right) L\left(\psi_{f}(g)^{-I}\right) d\left(\psi_{f}(g), \psi_{f^{\prime}}(g)\right)<$ 


$$
\left\langle a\left(\varphi_{f}(g), \varphi_{f^{\prime}}(g)\right)+d\left(\psi_{f}(g), \psi_{f^{\prime}}(g)\right) .\right.
$$

Mas :

$a\left(\varphi_{f}(g), \varphi_{f}(g)\right)=d\left(f_{2} \circ(I, g), f_{2}^{\prime} \circ(I, g)\right) \leqslant d\left(f_{2}, f_{2}^{\prime}\right)$

$$
\text { e } d\left(\psi_{f}(g), \psi_{f}(g)\right)=d\left(f_{I} \circ(I, g), f_{I}^{\prime} \circ(I, g)\right) \leqslant d\left(f_{I}, f_{I}^{q}\right)
$$

Lo go,

$$
a\left(\Gamma_{f}(g), \Gamma_{f}(g)\right)<a\left(f_{2}, f_{2}^{\prime}\right)+a\left(f_{1}, f_{1}^{\prime}\right) \leqslant 2 . d\left(f, f^{\prime}\right)
$$

Então:

$$
\begin{aligned}
& d\left(g_{f}, g_{f^{\prime}}\right)=d\left(\Gamma_{f}\left(g_{f}\right), \Gamma_{f^{\prime}}\left(g_{f^{\prime}}\right)\right) \leqslant \\
& d\left(\Gamma_{f}\left(g_{f}\right), \Gamma_{f^{\prime}}\left(g_{f}\right)\right)+d\left(\Gamma_{f^{\prime}}\left(g_{f}\right), \Gamma_{f^{\prime}}\left(g_{f^{\prime}}\right)\right)< \\
& 2 d\left(f, f^{\prime}\right)+L\left(\Gamma_{f}\right), d\left(g_{f}, g_{f^{\prime}}\right)
\end{aligned}
$$

Donde, pondo $C=I\left(\Gamma_{f}\right)<I$,

$$
a\left(g_{f}, g_{f},\right)<\frac{2}{I \ldots C}, d\left(f, f^{\prime}\right)
$$

- đe mostra que a função $f \longmapsto g_{f}$ é uniformemente contínua.

$$
\text { Suponhamos agora que vale a proposição para } k-1 \geqslant 0 \text { e }
$$
mostremos que vale para $k$. Por 4.5 , sabemos que existe $j \in \mathbb{N}$ tal que 
(4-VII $) \quad g_{f}=f_{2}^{j} \circ\left(I, g_{\tilde{I}}\right) \circ\left[f_{1}^{j} \circ\left(I, g_{\tilde{I}}\right)\right]^{-I} / E_{I}(r)$

( as notações são as de 4.7 ). Seja então $\left(f_{n}\right)$ uma sequênci a de funções de $\mathbb{N}_{\varepsilon}^{\mathrm{k}}(\mathrm{T})$, com $f_{\mathrm{n}} \stackrel{\mathrm{C}^{\mathrm{k}}}{\longrightarrow} f \in \mathbb{N}_{\varepsilon}^{\mathrm{k}}(\mathbb{T})$. Existe $\mathbb{M}>0$ tal que $|f|_{k} \leqslant \mathbb{M}$ e $\left|f_{n}\right|_{k} \leqslant \mathbb{M}, \quad \forall n \in \mathbb{N}$, e portanto po demos tomar o mesmo $\tilde{r}$ para $f$ e para tôdas as $f_{n}$. Então :

$$
\mathrm{f}_{\mathrm{n}} \stackrel{\mathrm{C}^{\mathrm{k}}}{\longrightarrow} \mathrm{f} \Longrightarrow \tilde{\mathrm{f}}_{\mathrm{n}} \stackrel{\mathrm{C}^{\mathrm{k}}}{\longrightarrow} \tilde{\mathrm{x}} \stackrel{(4.6)}{\longrightarrow} \tilde{\mathrm{f}}_{\mathrm{n}}^{*} \stackrel{\mathrm{C}^{\mathrm{k}-1}}{\longrightarrow} \tilde{\mathrm{I}}^{*}
$$

Pela hipótese de indução ( lembremos que $\left.\tilde{f} \in \mathbb{N}_{\tilde{\varepsilon}}^{\mathrm{k}-I}(T \times T)\right)$, segue-se que

$$
g_{\tilde{f}_{n}^{*}} \stackrel{\mathrm{c}^{\mathrm{k}-1}}{\longrightarrow} \mathrm{g}_{\tilde{\mathrm{I}}^{*}}
$$

donde

$$
\mathrm{g}_{\tilde{\mathrm{f}}_{\mathrm{n}}^{*}}^{*} \stackrel{\mathrm{C}^{\mathrm{k}-1}}{\longrightarrow} \mathrm{g}_{\tilde{\mathrm{f}}}^{*}
$$

( vide final da demonstração de 4.7 )

Agora, do lema 4.6 segue-se que

$$
\mathrm{g}_{\tilde{I}_{n}} \stackrel{\mathrm{c}^{\mathrm{K}}}{\longrightarrow} \mathrm{g}_{\tilde{\mathbf{I}}}
$$


Aplicando (4-VII) para $g_{f_{n}}$, obtemos

$$
g_{f_{n}}=\left(f_{n}^{j}\right)_{2} \circ\left(I, g_{\tilde{f}_{n}}\right) \circ\left[\left(f_{n}^{j}\right)_{I} \circ\left(I, g_{\tilde{f}_{n}}\right)\right]^{-I} / E_{I}(r)
$$

donde

$$
g_{f_{n}} \stackrel{C^{k}}{\longrightarrow} f_{2}^{j} \circ\left(I, g_{\tilde{f}}\right) \circ\left[f_{1}^{j} \circ\left(1, g_{\tilde{f}}\right)\right]^{-1} / E_{1}(r)
$$

ou seja

$$
g_{f_{n}} \stackrel{c^{k}}{\longrightarrow} g_{f}
$$

4.10 TEOREMA. ( da Variedade Instável )

Seja $E$ um espaço de Banach e $T: E \longrightarrow E$ um operador hiperbólico com assimetria $G$. Dado $r>0$, existem $\hat{E}>0$ independente de $r$ e $S>0$ tais que se $f: \mathbb{E}(r) \longrightarrow \mathbb{E}$ é uma função lipschitziana com $\mathrm{I}(\mathrm{f}-\mathrm{T})<\mathcal{E}$ e $\left\|_{\mathrm{f}}(0)\right\|<S$, então existe uma única contração $\mathrm{g}_{\mathrm{f}}: \mathrm{E}_{1}(r) \longrightarrow \mathbb{E}_{2}(r)$ cujo gráfico é $\quad W_{I}=\bigcap_{n \geqslant 0} f^{n}(E(r)) ;$ mais ainda, se $f \in U c^{K}$, então $g_{f} \in C^{k}$ (uniformemente em uma bola $E(\hat{r})$ com $\hat{r} \leqslant r$ ); a função $f \longmapsto g_{f}, d e$

$\mathbb{N}_{\varepsilon, \delta}^{\mathrm{K}}(\mathbb{T})=\left\{f \in \mathrm{UC}^{\mathrm{k}}(\mathbb{E}(r), \mathbb{E}): L(f-\mathbb{T})<\varepsilon\right.$ e $\left.\quad\|f(0)\|<S\right\}$ em $\quad C^{k}\left(E_{1}(r), E_{2}(r)\right)$ é contínua; e $e^{-1} / W_{1}: W_{1} \longrightarrow W_{1}$ é uma 
Demonstração :

Afirmamos que basta tomar

$\varepsilon<\min \left\{\frac{1-\zeta}{1+\sigma},\left\|T^{-1}\right\|^{-1}\right\} \quad \delta<\frac{\varepsilon^{2} r \sigma}{1+\sigma} . \quad$ se

$f(0)=0$, o teorema é verdadeiro, como vimos em $4.1,4.2,4.4$, 4.7, e 4.9. Suponhamos então $f(0) \neq 0$. Mostraremos nêste caso que $f$ tem um ponto fixo em $E(r)$ e faremos uma "translação " da origem para êsse ponto, recaindo no caso já demonstrado.

$$
\begin{aligned}
\text { Tomemos } & \bar{r}<r \text { tal que } S<\bar{r} \varepsilon^{2} \zeta \text { edefinamos } \\
& \overline{\mathrm{r}}: \overline{\mathrm{E}(\overline{\mathrm{r}})} \longrightarrow \mathrm{E}
\end{aligned}
$$$$
\text { por } \quad I(x, y)=\left(T_{1}^{-1}\left(T_{1} x+x-f_{1}(x, y)\right), f_{2}(x, y)\right)
$$

Então :

(i) $\vec{f}$ e $f$ têm os mesmos pontos fixos

De fato:

$$
\begin{aligned}
\mathrm{I}(\mathrm{x}, \mathrm{y})= & (\mathrm{x}, \mathrm{y}) \Longleftrightarrow \mathrm{T}_{1} \mathrm{x}=\mathrm{T}_{1} \mathrm{x}+\mathrm{x}-\mathrm{f}_{1}(\mathrm{x}, \mathrm{y}) \quad \text { e } f_{2}(\mathrm{x}, \mathrm{y})=\mathrm{y} \\
& \Longleftrightarrow \mathrm{f}_{1}(\mathrm{x}, \mathrm{y})=\mathrm{x} \text { e } \mathrm{f}_{2}(\mathrm{x}, \mathrm{y})=\mathrm{y} \Longleftrightarrow f(x, y)=(x, y)
\end{aligned}
$$

(ii) $\mathbf{I}$ é uma contração.

Realmente: $\operatorname{se}(x, y),(a, b) \in \overline{E(\bar{r})}$, 
78

$$
\begin{aligned}
& \|I(x, y)-f(a, b)\|=\max \left\{\| T_{I}^{-1}\left(T_{1} x+x-f_{I}(x, y)-\right.\right. \\
& \left.-T_{I}^{-1}\left(T_{I} a+a-f_{I}(a, b)\right)\|\|_{1} f_{2}(x, y)-f_{2}(a, b) \|\right\}
\end{aligned}
$$

Mas :

$$
\begin{aligned}
& \left\|T_{1}^{-1}\left(T_{1} x+x-f_{1}(x, y)\right)-T_{1}^{-1}\left(T_{1} a+a-f_{1}(a, b)\right)\right\| \leqslant \\
& \leqslant\left\|T_{1}^{-1}\right\| \cdot\left\|T_{1} x-T_{1} a-\left[f_{1}(x, y)-f_{1}(a, b)\right]+(x-a)\right\| \leqslant \\
& \leqslant \delta\left\|\pi_{I}[(T-f)(x, y)-(T-f)(a, b)]+(x-a)\right\| \leqslant \\
& \leqslant \zeta \cdot \varepsilon\|(x, y)-(a, b)\|+\zeta\|x-a\| \leqslant \\
& \leqslant(z \varepsilon+z)\|(x, y)-(a, b)\|
\end{aligned}
$$

e par outro lado

$$
\left\|f_{2}(x, y)-f_{2}(a, b)\right\| \leqslant(\zeta+\varepsilon)\|(x, y)-(a, b)\| \quad(\text { lema } 3.2)
$$

Segue-se que

$$
\|\bar{f}(x, y)-\bar{f}(a, b)\| \leqslant(z+\varepsilon)\|(x, y)-(a, b)\|
$$

e portanto: $I(\overline{\mathrm{I}}) \leqslant \Xi+\varepsilon<1$. 
(iii) $\overline{\mathrm{f}}(\overline{\mathrm{E}(\overline{\mathrm{r}})} \subset \overline{\mathrm{E}(\bar{r})}$

De fato: fazendo $(a, b)=(0, a)$ em (ii) obtemos, para todo $(x, y) \in \overline{E(\bar{r})}$ :

$$
\|\bar{I}(x, y) \ldots \bar{I}(0,0)\| \leqslant(\bar{\zeta}+\varepsilon)\|(x, y)\| \leqslant(\bar{\zeta}+\varepsilon) \bar{r}
$$

Então:

$$
\|\mathrm{I}(\mathrm{x}, \mathrm{y})\| \leqslant\|\mp(0,0)\|+(\bar{\zeta}+\varepsilon) \bar{r}
$$

Por outro lado,

$$
\begin{aligned}
& \left.\|I(0,0)\|=\| T_{1}^{-1}\left(-f_{1}(0,0)\right), f_{2}(0,0,)\right) \|= \\
& =\max \left\{\left\|T_{1}^{-1}\left(f_{1}(0,0)\right)\right\|,\left\|f_{2}(0,0)\right\| \leqslant \leqslant\right.
\end{aligned}
$$$$
\leqslant \max \left\{\zeta\left\|f_{1}(0,0)\right\|,\left\|f_{2}(0,0)\right\|\right\} \leqslant\|f(0,0)\|<\delta
$$

Loge, $\quad\|\mathrm{I}(\mathrm{x}, \mathrm{y})\|<\delta+(\zeta+\varepsilon) \bar{r}<\varepsilon^{2} \overline{\mathrm{r}} \zeta+(\bar{\zeta}+\varepsilon) \bar{r}=$

$$
=\bar{r}\left(\varepsilon^{2} \circlearrowright+\bar{\zeta}+\varepsilon\right)<\vec{r}\left(\varepsilon^{2} \zeta+1-\bar{\zeta}\right)=\bar{r}(1-\zeta \varepsilon(1-\varepsilon))<\bar{r}
$$

Concluimos que $\bar{f}$, e portanto $f$, tem um único ponto fi xo $p_{f}=\left(p_{1}, p_{2}\right) \in \overline{E(\bar{r})} \subset E(r)$. Fazendo $g=T / \overline{E(\bar{r})}$ na proposição 1.3, concluímos ainda que 
80

$$
\left\|p_{f}\right\|<\frac{\delta}{1-\sigma-\varepsilon}
$$

Seja agora

$$
\hat{r}=r-\delta(1-Z-\xi)^{-1}<r-\left\|p_{f}\right\|
$$

Uma vez que

$$
\begin{aligned}
& r-\int(1-\zeta-\varepsilon)^{-1}>r-\frac{\varepsilon^{2} r \zeta}{(1+\zeta)(1-\zeta-\varepsilon)}>r-\frac{\varepsilon^{2} r \zeta}{1-\zeta-\varepsilon}= \\
& \quad=r \frac{1-\zeta-\varepsilon-\varepsilon^{2} \zeta}{1-\zeta-\varepsilon}
\end{aligned}
$$

e como

$$
1-\zeta-\varepsilon-\varepsilon^{2} z>\zeta \varepsilon-\varepsilon^{2} \vec{\zeta}=\zeta \varepsilon(1-\varepsilon)>0
$$

e $\quad 1=\measuredangle-\varepsilon>0$,

temos $\hat{r}>0$. Definamos então $\hat{f}: E(\hat{r}) \longrightarrow E$

por

$$
\hat{f}(x)=f\left(x+p_{f}\right)-p_{f}
$$

Note-se que

$$
\|x\|<\hat{r} \Rightarrow\left\|x+p_{f}\right\| \leqslant\|x\|+\left\|p_{f}\right\|<\hat{r}+\left\|p_{f}\right\|=r
$$

- que garante que $\hat{f}$ está bem definida. Além aiso, $\hat{f}(0)=0$ e

$$
I(\hat{f}-T) \leqslant I(f-T)<\varepsilon
$$




$$
\begin{aligned}
& \text { pois }\|(\hat{f}-T)(x)-(\hat{f}-T)(y)\|= \\
& =\left\|f\left(x+p_{f}\right)-p_{f}-T_{x}-f\left(y+p_{f}\right)+p_{f}+T_{y}\right\|= \\
& =\left\|(f-T)\left(x+p_{f}\right)-(f-T)\left(y+p_{f}\right)\right\| \leqslant I(f-T)\|x-y\|
\end{aligned}
$$

Concluímos que existe uma função $\hat{g}=g_{\hat{f}}: E_{1}(\hat{r}) \longrightarrow E_{2}(\hat{r})$, satisfazendo ao enunciado do teorema. A partir de $\hat{g}$, definimos a função

$$
g=g_{f}: E_{1}(r) \longrightarrow E_{2}(r)
$$

tal que

$$
g(x)=p_{2}+\varphi_{\hat{f}}(\hat{g}) \circ \psi_{\hat{f}}(\hat{g})^{-1}\left(x-p_{1}\right)
$$

( então: $\left.g\left(p_{1}\right)=p_{2}\right)$. Mostremos que $g$ está bem definida em $\mathrm{E}_{1}(r)$ e toma realmente valores em $\mathrm{E}_{2}(r)$. Inicialmente, afirmamos que

$$
\delta<\frac{\left(\gamma^{-1}-\varepsilon-1\right)(1-\zeta-\varepsilon) r}{1+z^{-1}-\varepsilon}
$$

De fato:

$$
\delta<\frac{\varepsilon^{2} r \zeta}{1+\zeta}<\frac{\left(\frac{1-\zeta}{1+\zeta}\right)^{2} r \zeta}{1+\zeta}=
$$


82

$$
\begin{aligned}
& =\frac{(1-\zeta)(1-\zeta) r}{(1+\zeta)^{2}\left(\zeta^{-1}+1\right)}=\frac{\left(\bar{\zeta}^{-1}-1\right)\left(\bar{\zeta}^{-1} \bar{\zeta}^{2}\right) r}{\left(1+\bar{\zeta}^{2}\left(1+\bar{\zeta}^{-1}\right)\right.}= \\
& =\frac{\left(\sigma^{-1}-\frac{1-\zeta}{1+\zeta}-1\right)\left(1-\zeta-\frac{1-\zeta}{1+z}\right) x}{1+\zeta^{-1}}< \\
& <\frac{\left(z^{-1}-\varepsilon-1\right)(1-\zeta-\varepsilon) r}{1+\zeta^{-1}-\varepsilon^{-\varepsilon}}
\end{aligned}
$$

Segue-se que

(4-VIII) $\quad\left(\sigma^{-I}-\varepsilon\right) \hat{r}>r+\delta(1-6-\varepsilon)^{-1}$

pois

$$
\begin{aligned}
& \left(\Xi^{-1}-\varepsilon\right) \hat{r}-r-\delta(1-Z-\varepsilon)^{-1}= \\
& \left.\quad=r\left(\zeta^{-1}-\varepsilon-1\right)-\frac{z^{-1}-\varepsilon+1}{1-\zeta-\varepsilon} \cdot \delta\right)
\end{aligned}
$$

$$
>r\left(\zeta^{-1}-\varepsilon-1\right)-\frac{\left(\zeta^{-1}-\varepsilon+1\right)\left(\zeta^{-1}-\varepsilon-1\right)(1-\zeta-\varepsilon) r}{(1-\zeta-\varepsilon)\left(1+\zeta^{-1}-\varepsilon\right)}=0
$$

Por outro lado, $\quad I\left(\psi_{\hat{f}}(\hat{g})^{-1}\right) \leqslant\left(\zeta^{-1}-\varepsilon\right)^{-1}$ 
$\psi_{\hat{f}}(\hat{g})(0)=0$ acarretam, por 1.11 :

$$
\psi_{\hat{f}}(\hat{g})\left(E_{I}(\hat{r})\right) \supset E_{I}\left(\left(\zeta^{-1}-\varepsilon\right) \hat{r}\right)
$$

Conclusão:

$x \in E_{1}(r) \Rightarrow\left\|x-p_{1}\right\| \leqslant\|x\|+\left\|p_{1}\right\| \leqslant\|x\|+\left\|p_{f}\right\| \leqslant$

$$
\begin{aligned}
& \leqslant r+\delta(1-\zeta-\varepsilon)^{-1} \leqslant\left(\zeta^{-1}-\varepsilon\right) \hat{r}= \\
& x-p_{1} \in E_{1}\left(\left(乙^{-1}-\varepsilon\right) \hat{r}\right) \Rightarrow x-p_{1} \in \psi_{\hat{f}}(\hat{g})\left(E_{1}(\hat{r})\right) \Longrightarrow \\
& \Longrightarrow \exists \psi_{\hat{f}}(\hat{g})^{-1}\left(x-p_{1}\right) \in E_{1}(\hat{r}) \\
& \stackrel{\mathrm{f}}{\Longrightarrow} \varphi_{\hat{\mathrm{f}}}(\hat{\mathrm{g}}) \circ \psi_{\hat{\mathrm{f}}}(\hat{\mathrm{g}})^{-1}\left(\mathrm{x}-\mathrm{p}_{1}\right) \quad \in \mathrm{E}_{2}(\hat{r}) \\
& \Longrightarrow\|g(x)\| \leqslant\left\|p_{2}\right\|+\hat{r} \leqslant\left\|p_{f}\right\|+\hat{r}<r
\end{aligned}
$$

Construída a função

$$
g: \mathrm{E}_{1}(r) \longrightarrow \mathrm{E}_{2}(r)
$$

devemos agora mostrar que ela satisfaz às condições do teorema :

$$
\begin{gathered}
(a) I(g)<1 \text {, pois } \\
\|g(x)-g(y)\| \leqslant I\left(\varphi_{\hat{f}}(\hat{g})\right) \cdot I\left(\psi_{\hat{f}}(\hat{g})^{-1}\right)\|x-y\|
\end{gathered}
$$


(b) $f($ graf $g) \cap \mathbb{E}(r)=\operatorname{graf} g$

Em primeiro lugar, mostremos que se $(x, g(x)) \in$ graf $g$ e $f(x, g(x)) \in E(r)$, então $\left\|x-p_{I}\right\|<\hat{r}$. De fato: como

I $\left(\psi_{f}(g)^{-1}\right) \leqslant\left(\zeta^{-1}-\xi\right)^{-I}$ ( a demonstração de 3.4 não utiliza a hipótese $\mathrm{g}(0)=0$.), temos:

$$
\begin{gathered}
\left\|\psi_{f}(g)(x)-p_{1}\right\|=\left\|\psi_{f}(g)(x)-\psi_{f}(g)\left(p_{1}\right)\right\| \Longrightarrow \\
\Longrightarrow\left\|x-p_{1}\right\| \leqslant\left(\zeta^{-1}-\varepsilon\right)^{-1}\left\|\psi_{f}(g)(x)-p_{1}\right\| \leqslant \\
\leqslant\left(\zeta^{-1}-\varepsilon\right)^{-1}\left[\left\|\psi_{f}(g)(x)\right\|+\left\|p_{1}\right\|\right]< \\
<\left(\zeta^{-1}-\varepsilon\right)^{-1}\left[r+\delta(1-\zeta-\varepsilon)^{-1}\right] \leqslant{ }^{(4-V I I I)} \\
\leqslant\left(\zeta^{-1}-\varepsilon\right)^{-1} \cdot\left(\zeta^{-1}-\varepsilon\right) \hat{r}=\hat{r}
\end{gathered}
$$

Então:

$g\left(f_{1}(x, g(x))=p_{2}+\varphi_{\hat{f}}(\hat{g}) \circ \psi_{\hat{f}}(\hat{g})^{-1}\left(f_{I}(x, g(x))-p_{I}\right)\right.$

Mas :

$$
f_{1}(x, g(x))-p_{1}=\hat{f}_{1}\left(x-p_{1}, g(x)-p_{2}\right)
$$




$$
e g(x)-p_{2}=\varphi_{\hat{f}}(\hat{g}) \circ \psi_{\hat{f}}(\hat{g})^{-1}\left(x \cdots p_{1}\right)=\hat{g}\left(x \cdots p_{1}\right)
$$

pois $\left\|x-p_{1}\right\|<\hat{r}$. Segue-se que

$$
\begin{aligned}
& g\left(f_{1}(x), g(x)\right)=p_{2}+\varphi_{\hat{f}}(\hat{g}) \circ \psi_{\hat{f}}(\hat{g})^{-1}\left(\hat{f}_{1}\left(x-p_{1}, \hat{g}\left(x-p_{1}\right)\right)\right)= \\
& =p_{2}+\varphi_{\hat{f}}(\hat{g}) \circ \psi_{\hat{f}}(\hat{g})^{-1} \circ \hat{f}_{1} \cap(1, \hat{g})\left(x-p_{1}\right)= \\
& =p_{2}+\varphi_{\hat{f}}(\hat{g})\left(x-p_{1}\right)=p_{2}+\hat{f}_{2}\left(x-p_{1}, \hat{g}\left(x-p_{1}\right)\right)= \\
& =f_{2}\left(x, \hat{g}\left(x-p_{1}\right)+p_{2}\right)=f_{2}(x, g(x))
\end{aligned}
$$

Isso mostra que

$$
f(\text { graf } g) \cap \mathbb{T}(r) \subset \text { graf } g
$$

Provemos agora a inclusão contrária : de (4-VIII), concluímos que existe $\tilde{r}<\hat{r}$ tal que $\left(乙^{-1}-\varepsilon\right) \tilde{r}>r+\delta(I-\circlearrowright-\varepsilon)^{-1}$. Dar por 1.11, temos

$$
\psi_{f}(g)\left(E_{1}\left(p_{1}, \hat{r}\right)\right)=E_{1}\left(p_{1},\left(\zeta^{-1} \ldots\right) \tilde{r}\right)
$$

( onde $E_{1}\left(p_{1}, \lambda\right.$ ) indica a bola aberta de centro $p_{1}$ e raio $\lambda$ ). Concluímos que

$$
E_{I}(r) \subset \psi_{f}(g)\left(E_{I}\left(p_{I}, \hat{r}\right)\right) 。
$$


Então, se $(y, g(y)) \in$ graf $g$, existe $x \in E_{I}\left(p_{1}, \hat{r}\right)$

( e portanto, $x \in E_{1}(r)$ ) tal que $y=\psi_{f}(g)(x)$.

Logo,

$$
f_{2}(x, g(x))=f_{2} c(I, g)(x)=\rho_{f}(g) \circ \psi_{f}^{t}(g)^{-1}(y)=g(y)
$$

donde

$$
(y, g(y))=f(x, g(x))
$$

e portanto

$$
\text { graf } g<f(\text { graf g) }
$$

- que acarrota

graf $g \in f$ (graf $g) \cap E(r)$

(c) graf g $=W_{1}$

( demonstração idêntica à de 4.1 )

(d) $f^{-1} / W_{I}: W_{I} \rightarrow W_{I}$ é uma contração. ( demonstração idêntica à de 4.2 )

(e) $f \in U C^{k} \Longrightarrow g \in C^{k}$

De fato: $f \in U C^{k} \Longrightarrow \hat{f} \in U C^{k} \stackrel{(4.7)}{\Rightarrow} \hat{g} \in C^{k} \Rightarrow g \in C^{k}$

(f) $f \mapsto g_{f}$ é uma função contínua de $\mathbb{N}_{\varepsilon, \delta}^{k}(T)$ em $C^{k}\left(E_{1}(r), E_{2}(r)\right)$ 
87

De fato:

$$
\begin{array}{r}
f_{n} \stackrel{c^{k}}{\longrightarrow} f \Longrightarrow \hat{f}_{n} \stackrel{c^{k}}{\longrightarrow} \hat{f} \stackrel{(4.9)}{\Rightarrow} g_{\hat{f}_{n}} \stackrel{c^{k}}{\longrightarrow} g_{\hat{f}} \Longrightarrow g_{f_{n}} \stackrel{c^{k}}{\longrightarrow} g_{f} \\
* * *
\end{array}
$$

4.II COROLARIO. O toorema da variedade instável vale para $k=\infty$, uma vez que $\varepsilon \in \delta$ não dependem de $k$.

4.12 COROLARIO. Se $f \in U^{k}(k \geqslant I)$, então $W_{I}$ é uma variedade de Banach modelada em $E_{1}$.

Demonstração :

Basta tomar o atlas constituído pela única carta

$$
\pi 1: W_{1} \longrightarrow E_{1}(r)
$$

$$
* * *
$$

4.13 COROLARIO. Seja E um espaço de Banach e $V \subset E$ uma vizinhança da origom. Seja $f: V \longrightarrow E$ um difeomorfismo de classe $C^{I}$ tendo a origem como ponto fixo hiperbólico ( isto ef, $f(0)=0$ e $D f(0)=T$ é um operador hiperbólico). Então, exis tem $\mathcal{E}, \mathcal{S}$, r para os quais valem as conclusões do teorema 4.10. Além disso, se $f \in U C^{I}$, tem-se $T_{0} W_{I}=E_{I}$ ( a menos de isomorfís mos ).

Demonstração: 
Como $D(f-T)(0)=D f(0)-T=0$, temos:

dado $\varepsilon<\min \left\{\frac{1-Z}{1+\zeta}, \quad\left\|T^{-1}\right\|^{-1}\right\}$, existe $E(r) \subset V$ tal que $I[(f-T) / E(r)]<\varepsilon \quad($ vide 1.13).

Segue-se que, $\forall d>0$, vale o teorema 4.10 .

Quanto à segunda parte, é suficiente mostrar que $\operatorname{Dg}_{f}(0)=0$, pois nesse caso a diferencial da " carta " $\left(1, g_{f}\right)$ na $\underline{0}$ rigem será $\left(i, D_{g_{f}}(0)\right)=(I, 0)$ e portanto, para todo $z \in E_{I}$,

$$
\left(1, \operatorname{Dg}_{f}(0)(x)=(x, 0)\right.
$$

ou seja: $\quad T_{0} W_{1}=E_{1} \quad$ Então:

$$
\begin{aligned}
& g_{f}=f_{2} \circ\left(I, g_{f}\right) \circ\left[f_{I} \circ\left(I, g_{f}\right)\right]^{-I} / E_{I}(r) \Longrightarrow \\
& \Longrightarrow D g_{f}(0)=D f_{2}(0) \circ\left(I, D g_{f}(0)\right) \circ\left[D f_{1}(0) \circ\left(I, D g_{f}(0)\right)\right]-I
\end{aligned}
$$

e portanto, restringindo à bola $E_{1}(I)$, obtemos por 3.7 :

$$
\mathrm{Dg}_{\mathrm{f}}(0)=\Gamma_{f}(0)\left(\mathrm{Dg}_{\mathrm{f}}(0)\right)=\Gamma_{\mathrm{T}}\left(\mathrm{Dg}_{\mathrm{f}}(0)\right)=\mathrm{T}_{2} \circ \mathrm{Dg}_{\mathrm{f}}(0) \circ \mathrm{T}^{-1}
$$

donde

$$
\left\|D g_{f}(0)\right\| \leqslant\left\|T_{2}\right\| \cdot\left\|D_{g_{f}}(0)\right\| \cdot\left\|T_{1}^{-1}\right\|
$$

Então, como $\left\|\mathrm{T}_{2}\right\|<I$ e $\left\|\mathrm{T}_{1}^{-1}\right\|<1, \quad \operatorname{Dg}_{\mathrm{f}}(0) \neq 0$ 
Ievaria ao absurdo $\left\|D g_{f}(0)\right\|<\left\|D g_{f}(0)\right\|$

$$
* * *
$$

4.14 TEOREMA. (da variedade estável). Seja E um es paço dẹ Banach e $T: E \rightarrow E$ um operador hiperbólico com assimetri a 6 . Dado $r>0$, existem $\varepsilon>0$ independente de $r$ e $\delta>0$ tais que se $f: E(r) \longrightarrow E$ é uma função lipschitzi ana tal que $I(f-T)<\varepsilon$ e $\|f(0)\|<\delta$, então existe uma única contração $g_{2 f}: E_{2}(r) \longrightarrow E_{1}(r)$ cujo gráfico é $W_{2}=n_{n \geqslant 0}^{n} f^{-n}(E(r))$; além disso: $f / W_{2}: W_{2} \longrightarrow W_{2}$ é uma contração: se $f \in U \mathrm{C}^{\mathrm{k}}$, então $g_{2 f} \in \mathrm{C}^{\mathrm{k}}$; e a função $\mathbb{N}_{\varepsilon, \delta}^{\mathrm{k}}(\mathrm{T}) \longrightarrow \mathrm{C}^{\mathrm{k}}\left(\mathrm{E}_{2}(\mathrm{r}), \mathrm{E}_{1}(\mathrm{r})\right)$ definida por $\mathrm{f} \longmapsto \mathrm{g}_{2 f}$ é continua.

Demonstração .

A idéia é mostrar que " a variedade estável para $f$ é a variedade instável para $f^{-1} "$. Surge então a dificuldade de $f^{-1}$ não estar definida em $E(r)$. Mostremos então que existe $\tilde{r}>0$ tal que $E(\tilde{r}) \in f(E(r))$. Tomemos inicialmente $\varepsilon<\min \left\{\frac{1-\zeta}{1+\zeta},\left\|T^{-1}\right\|^{-1}\right\} \quad$ e $\quad \delta<\frac{\varepsilon^{2} r \zeta}{1+\zeta}$ Então, pelo que foi visto na demonstração do teorema 4.10, concluÍ mos ( com as notações lá utilizadas) que $\overline{\mathrm{E}\left(\mathrm{p}_{\mathrm{f}}, \rho\right)} \subset \mathrm{E}(\mathrm{r}), \forall \rho<\hat{r}$ donde, por 1.II, $E\left(p_{f},\left(\sigma^{-1}-\varepsilon\right) \rho\right) \subset f(E(r)), \forall \rho<\hat{r}$. Além disso, 
90

de $\delta<\frac{\varepsilon^{2} r \zeta}{1+\zeta} \quad$ segue $\left(\zeta^{-1}-\varepsilon\right) \hat{r}>\delta(1-\zeta-\varepsilon)^{-1}$ (v. 4.VIII) - Tomando então $\rho$ tal que

$$
\begin{aligned}
& S(1-\zeta-\varepsilon)^{-1}<\left(\zeta^{-1}-\varepsilon\right) \rho<\left(\zeta^{-1}-\varepsilon\right) \hat{x} \text {, temos: } \\
& \left\|p_{1}\right\|<\delta(1-\zeta-\varepsilon)^{-1}<\left(\zeta^{-1}-\varepsilon\right) \rho \text { donde }
\end{aligned}
$$

$0 \in E\left(p_{f},\left(\zeta^{-1}-\varepsilon\right) \rho\right) \subset f(\mathbb{E}(r))$. Daŕ, como $f(E(r))$ é aberto (Teorema 1.10 ), existe $\tilde{r}>0$ tal que $E(\tilde{r}) \subset f(E(r))$ e portanto $f^{-1}$ está definida em $E(\tilde{r})$.

Por outro lado, o operador $S=T^{-1}: \mathbb{H} \longrightarrow \mathbb{E}$ é evi dentemente hiperbólico ( $\lambda$ é valor espectral de $\mathrm{T} \Leftrightarrow \lambda^{-1}$ é va lor espectral de $\mathrm{T}^{-1}$ ). Seja $E=F_{I} \Theta \mathbb{F}_{2}$ a decomposição canônica de $E$ relativamente a $S$ - Então, uma vez que

$$
\left\|S / E_{1}\right\|<1 \text { e }\left\|S^{-1} / E_{2}\right\|<1 \text {, concluímos que } F_{1}=E_{2}
$$

e $F_{2}=E_{1}$ e que $S$ tem a mesma assimetria $(\zeta)$ que $T$. Va mos mostrar agora que $f^{-1}: E:(\tilde{r}) \longrightarrow E$ satisfaz, relativamente a $\mathrm{S}$, às hipóteses do teorema 4.10 ( a menos de uma escolha conveni ente de $\xi e \delta$ ) . Em primeiro lugar, de

$$
f^{-1}-S=S T f^{-1}-S f f^{-1}=S \circ(T-f) 0 f^{-1} \text {, e de } 1.10 \text {, se- }
$$

gue-se que

$I\left(f^{-1}-S\right) \leqslant\|S\| \cdot I(f \cdots T) \cdot I\left(f^{-1}\right) \leqslant\|S\| L(f-T)\left[\|S\|^{-1}-L(f-T)\right]^{-1}$ Isso mostra que dado $\eta>0, \eta<\min \left\{\frac{1-\zeta}{1+\zeta},\left\|\mathrm{s}^{-1}\right\|^{-1}\right\}$, 
existe $\varepsilon_{1}>0$ tal que $L(f-T)<\varepsilon_{I} \Longrightarrow I\left(f^{-1}-S\right)<\eta$. Por outro lado, aplicando novamente 1.10 , temos: $\left\|f^{-1}(0)\right\|_{1}^{\prime} \leqslant f^{-1}(0)-f^{-1}\left(p_{f}\right)\|+\| p_{f} \| \leqslant$ $\leqslant\left[I+I\left(f^{-1}\right)\right]\left\|p_{f}\right\| \leqslant\left\{1+\left[\|S\|^{-1}-I(f-T)\right]^{-1}\right\} \delta(I-\sigma-\varepsilon)^{-1}$ Concluímos que, dado $\mu>0, \quad u<\frac{\eta^{2} \tilde{r} b}{1+\vec{b}}$, existem $\varepsilon_{2}$ e $\delta_{I}$ tais que $\mathrm{L}(\mathrm{f}-\mathrm{T})<\varepsilon_{2}$ e $\delta<\delta_{1} \Longrightarrow\left\|f^{-1}(0)\right\|<\mu$. Tomemos agora $\varepsilon<\min \left\{\varepsilon_{1}, \varepsilon_{2}, \frac{1-\sigma}{1+\zeta},\left\|\mathrm{T}^{-1}\right\|^{-1}\right\}$ $\cdot \delta<\min \left\{\frac{\varepsilon^{2} r \zeta}{1+\zeta}, \delta_{1}\right\}$

Então, para tôda função $f: E(r) \rightarrow E$, lipschitziana, tal que $I(f-T)<\varepsilon$ e $\|f(0)\|<\delta$, existe $\tilde{r}>0$ tal que $f^{-1}: E(\tilde{r}) \longrightarrow E$ está bem definida, é lipschitziana, com $I\left(f^{-1}-S\right)<\eta<\min \left\{\frac{1-\zeta}{1+\zeta}, \quad\left\|S^{-1}\right\|^{-1}\right\}$ e $\left\|\mathrm{f}^{-1}(0)\right\|<\mu<\frac{m^{2} \tilde{r} \zeta}{1+\zeta}$. Logo, valem para $f^{-1}$ as conclusões do teorema 4.10 (com M e $\mu$ representando os papéis de $\mathcal{E}$ e $\delta$, respectivamente). Como já se fêz na demonstração do lema 4.5 , podemos provar que existe $j \in \mathbb{N}$ tal que $f^{-j}\left(W_{2}(f / E(\tilde{r}))\right) \cap E(r)=W_{2}(f)$, Mas, evidentemente, $f^{-j}\left(W_{2}(f / E(\tilde{r}))\right)=f^{-j}\left(W_{1}\left(f^{-I} / E(\tilde{r})\right)\right)$ donde $W_{2}(f)=f^{-j}\left(W_{1}\left(f^{-1} / E(\tilde{r})\right)\right) \quad$ o que completa a demonstração (os detalhes são deixados ao leitor). 


\section{5 - OBSERVAÇÕES FINAIS}

5.1 Seja $T: E \rightarrow E$ hiperbólico e $f: E \rightarrow E$ tal que na bola $E(r)$ se tenha $I(f-T)<\varepsilon<\min \left\{\frac{1-\zeta}{1+\zeta},\left\|T^{-1}\right\|^{-1}\right\}$, $\operatorname{com}\|f(0)\|<\varepsilon<\frac{\varepsilon^{2} r \zeta}{1+\zeta} \quad$ os teoremas 4.10 e 4.14 garantem a existência das variedades instável ( $\left.W_{1}\right)$ e estável $\left(W_{2}\right)$ locais, isto $e$, em $E(r)$. Por iteração de $f$ sôbre $W_{1}$ e de $f^{-1}$ sôbre $W_{2}$, obtemos as variedades globais ( que podem não ser gráficos de funções ) .
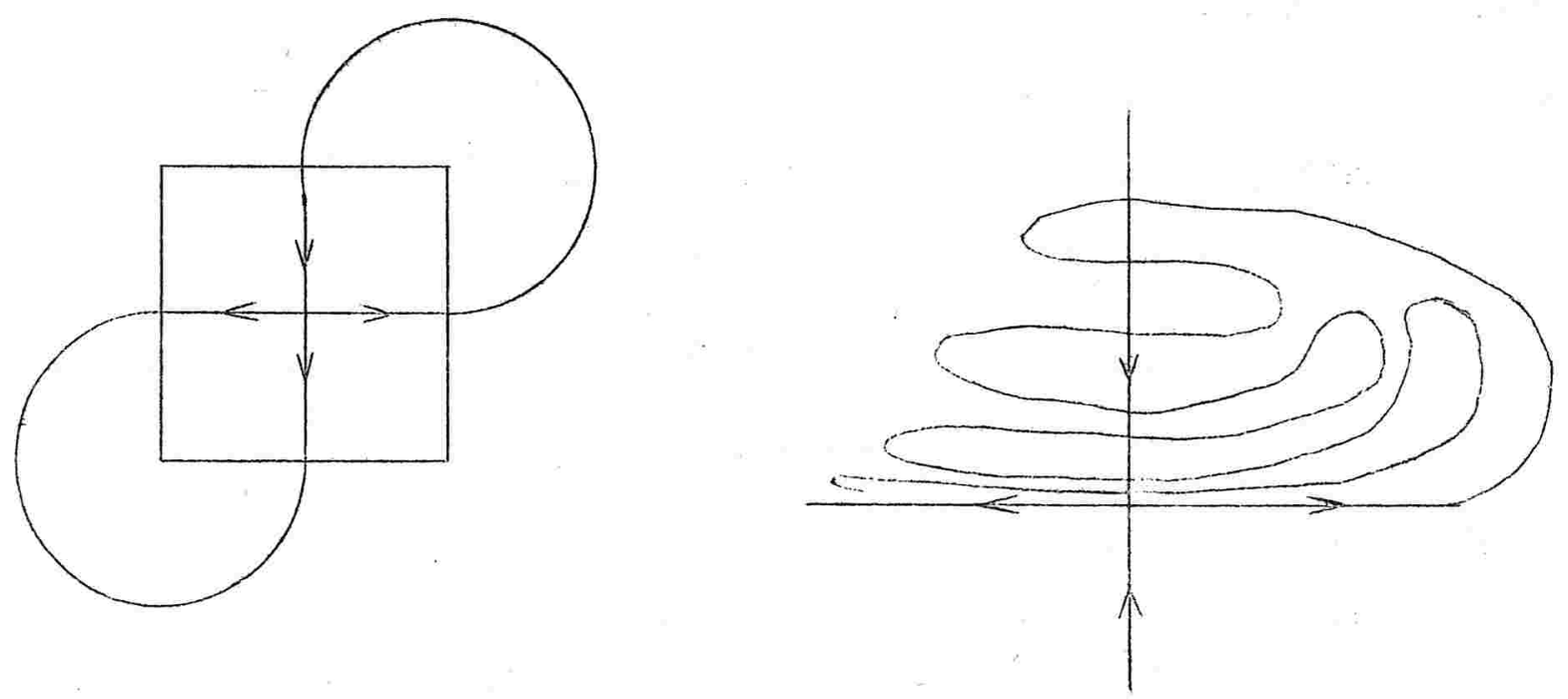

5.2 A generalização da proposição 4.9 para $r=\infty$ apre senta a seguinte dificuldade: quando $\|x\| \rightarrow \infty$, o mesmo sucede com o número $j$ de iterações de $f^{-1}$ necessário para trazer $\left(x, g_{f}(x)\right)$ para a bola $E(\tilde{r})$. Tome-se como exemplo para cada $n \in \mathbb{N}, n \geqslant 4$, 
$f_{n}: \mathbb{R}^{2} \rightarrow \mathbb{R}^{2}$ tal que $f_{n}(x, y)=\left(2 x+\frac{y}{n}, \frac{y}{2}\right)$

$T: \mathbb{R}^{2} \rightarrow \mathbb{R}^{2}$ tal que $T(x, y)=(2 x$
$\zeta=\frac{1}{2},\left\|T^{-1}\right\|^{-1}=\frac{1}{2}$ e portanto

$I\left(f_{n}-T\right)=\|f-T\|=\frac{I}{n}<\varepsilon<\frac{I}{3}=\min \left\{\frac{1-6}{1+\sigma},\left\|T^{-1}\right\|^{-1}\right\}$

Isso quer dizer que as funções $f_{n}$ pertencem a $\mathbb{N}_{\varepsilon}^{k}(T)$ e convergem para $T$ na topologia $c^{k}, \forall k \geqslant 0$. Acontece que $g_{f_{n}}(x)=\frac{2 x}{3 n}$ (pois os auto-valores de $f$ são 2 e $\frac{1}{2}$, e o auto-espaço cor respondente ao auto-valor 2 é a reta 3 ny $-2 x=0$ ),

$g_{T}(x)=0 \quad \forall x \in \mathbb{R}$, e a sequência $\left(g_{f_{\underline{n}}}\right)$ não converge na topo logia $C^{O}$ para a função nula:

$$
\sup _{\mathrm{x}} \mathrm{R}\left|\mathrm{g}_{\mathrm{f}_{\mathrm{n}}}(\mathrm{x})\right|=\infty, \forall i n \in \mathbb{N}
$$

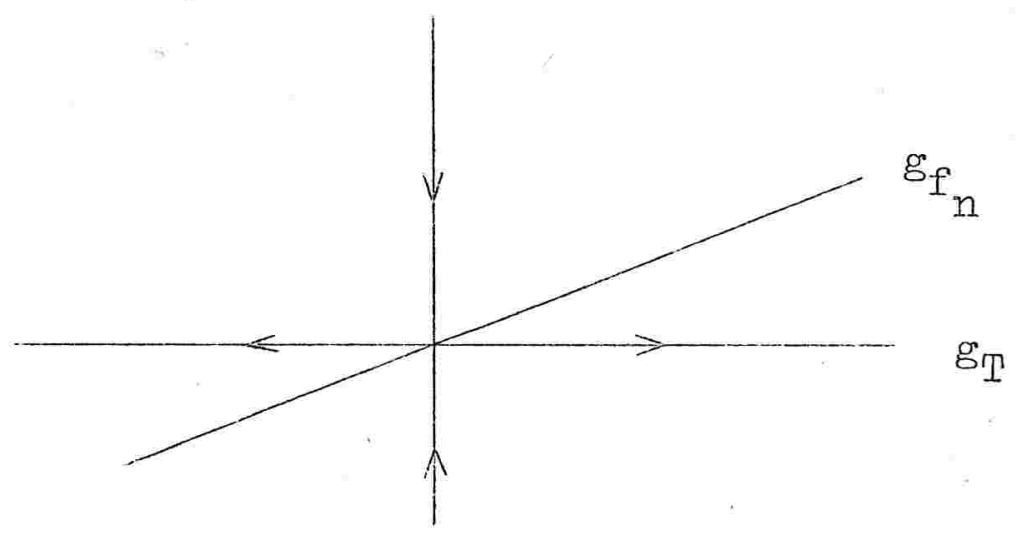

Em [6], os autores opinam que uma outra demonstração, com uma hipótese de indução diferente, possa ser imaginada. 
5.3 Se $f(0) \neq 0$, a funcão $\Gamma_{f}$ não leva necessáriamente elementos de $G$ em elcmentos de $G$. Ainda em [6] afirmam os autores que é possível demonstrar diretamente o teorema4.10 , acrescentando a $G$ funções $g$ tais que $g(0) \neq 0$ com $\|g(0)\|$ convenientemente, pequeno, e provando que $\Gamma_{f}$ é uma contração bem definida, etc.

5.4 Vejamos a seguir, a título ce exemplo de construcão de um operador hiperbólico, umą outra demonstração de que as funções, $\omega_{g}: H \longrightarrow H$ de 4.4 estão bem definidas e são contrą §ões. Ponhamos $Z=L(E)$ e $z_{i}=I\left(E, \mathbb{E}_{i}\right) \quad(i=I, 2)$ : E claro que $z=z_{1} \oplus z_{2}$ e que $z, z_{1}$ e $z_{2}$ são espaços de Banach - Usaremos a seguinte notação: se $S=S_{1} \oplus S_{2} \in Z$, e $\overline{\mathrm{S}} \in \mathrm{L}\left(\mathrm{E}_{1}, \mathrm{E}_{2}\right)$, então

$S^{\#}: z(1) \rightarrow z, \bar{S}^{\#}: z_{1}(1) \rightarrow z_{2}$ e $S_{i}^{\#}: z(1) \rightarrow z_{i}(i=1,2)$ indicam as composições à esquerda respectivamente por $S, \bar{S} e$ $S_{i}(i=1,2)$, isto é,

$$
\begin{aligned}
& S^{\#}(u)=S \circ u, \forall u \in z^{\prime}(I) \\
& S^{\#}(u)=\bar{S} \circ u, \forall u \in z_{I}(I) \\
& S_{i}^{\#}(u)=S_{i} \circ u, \forall u \in Z(I)(i=I, 2)
\end{aligned}
$$

Poremos ainda

$\left\|S^{\#}\right\|=\sup _{u \in Z(s)}\left\|S^{\#(1)}\right\|,\left\|S^{\#}\right\|=\sup _{u \in Z_{I}(1)}\left\|S^{\#}(u)\right\| e$ $\left\|S_{i}^{\#}\right\|=\sup _{u \in Z(1)}\left\|S_{i}^{\#}(x)\right\| \quad(i=1,2)$ 
Valem as seguintes propriedades, cujas demonstrações dei xamos a cargo do leitor:
(a)
$\left\|J^{\#}\right\|=\|J\|$
$\left(J=S, \bar{S}, S_{I}, S_{2}\right)$
(b) $\quad I_{E}^{\#} \quad=I_{Z(I)}$
(c) $\quad\left(S_{i}\right)^{\#} \quad=\left(S^{\#}\right)_{i} \quad(i=1,2)$
(d) $(S \circ \bar{S})^{\#}=S^{\#} \circ \bar{S}^{\#}, \forall \bar{S} \in Z$
(e) $S$ é inversível $\Longleftrightarrow S^{\#}$ é inversível e $\left(S^{\#}\right)^{-1}=\left(S^{-1}\right)^{\#}$
(f) $(s+\bar{S})^{\#}=S^{\#}+\bar{S}^{\#} \quad e(\lambda s)^{\#}=\lambda \cdot S^{\#}, \forall \bar{s} \in z, \forall \lambda \in R$
(g) $S^{\#}$ é Iipschitziana e $I\left(S^{\#}\right) \leqslant\|S\|$

(h) T \# (estendido a $z$ por linearidade) é um operador hiperbólico, deixa invariantes $z_{1}$ e $z_{2}$ e $z_{\Delta}=z_{1} \oplus z_{2}$ é a de composição canônica de, $Z$ em relação a $T^{\#}$ ( e portanto $T^{\#}$ tem assimetria 乙 ) .

Então: se $z \in E(r)$, restringindo-nos a $Z(1)$,

temos:

$I\left(D f(z)^{\#}-T^{\#}\right) \stackrel{(f)}{\leqslant} I\left((D f(z)-T)^{\#}\right) \stackrel{(g)}{\leqslant}\|D f(z)-T\|<\varepsilon$. $<\min \left\{\frac{1-\zeta}{1+\zeta},\left\|\left(T^{\#}\right)^{-1}\right\|^{-1}\right\} \quad$. Isso permite definir a contração $\Gamma_{D f(z)^{\#}: G}: G^{\#}=\left\{\theta \in \mathbb{M}\left(Z_{1}(I), \overline{Z_{2}(I)}\right): L(\theta) \leqslant 1\right\}$

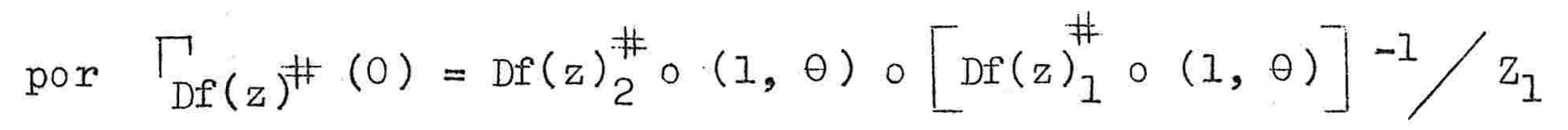
Mostremos agora que se $h \in \mathbb{H}$, então $h\left(z_{I}\right)^{\#} \in G^{\#}$ e

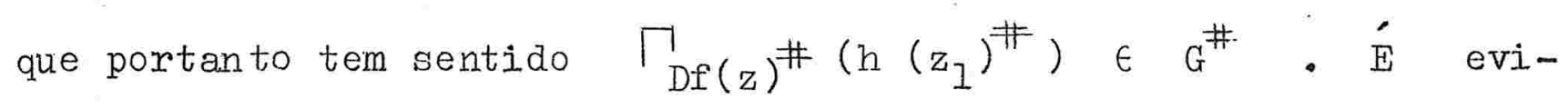


dente que $h\left(z_{1}\right)^{\#}(0)=0$ e que $h\left(z_{1}\right)^{\#}: z_{1}(I) \rightarrow z_{2}(I)$ $\left(\right.$ pois $\| h\left(z_{1}\right)$ \# $\left.(u)\|=\| h\left(z_{1}\right)^{\#} \circ u\|\leqslant\| h\left(z_{1}\right)\|\| u \| \leqslant l, \forall u \in z_{1}(I)\right)$ Quanto à constante de Lipschitz, basta observar que se $u, k \in z_{I}(I)$,

$\left\|h\left(z_{1}\right) \#(u)-n h\left(z_{1}\right) \#(k)\right\|=\left\|h\left(z_{1}\right) \circ u-h\left(z_{1}\right) \circ k\right\| \leqslant$

$\leqslant\left\|h\left(z_{I}\right)\right\|\|u-k\| \leqslant\|u-k\|$

Usando agora as propriedades (a) a (h), pode-se pro-

var que

$$
w_{g}(h)(x)=\left[\Gamma_{D f}(z)^{\#}\left(h\left(z_{1}\right)^{\#}\right)\left(\pi_{I}\right)\right] / E_{I}, \forall h \in H_{3}, \forall x \in E_{I}(x)
$$

donde sai que as funções $\omega_{\text {ğ }}$ são contrações: pondo

$\lambda=I\left(\Gamma_{\mathrm{Df}}(\mathrm{z})^{\#)}<1\right.$, temos:

$\left\|w_{g}\left(h_{1}\right)-w_{g}\left(h_{2}\right)\right\|=\sup _{x \in E_{1}(r)}\left\|w_{g}\left(h_{1}\right)(x)-w_{g}\left(h_{2}\right)(x)\right\|=$

$=\sup _{x \in \mathbb{E}_{1}(r)}\left\|\Gamma_{D \Gamma(z)}^{\#}\left(h_{1}\left(z_{1}\right)^{\#}\right)\left(\pi_{I}\right) / E_{1}-\Gamma_{D f(z)} \#\left(h_{2}\left(z_{1}\right)^{*}\right)\left(\pi_{1}\right) / E_{1}\right\|$

$=\sup _{x \in \mathbb{E}_{1}(r)}\left\|\left[\Gamma_{D f(z)}^{\#}\left(h_{1}\left(z_{1}\right)^{\#}\right)-\Gamma_{D f}(z)^{\#}\left(h_{2}\left(z_{1}\right)^{\#}\right)\right]\left(\pi_{1}\right) / \mathbb{E}_{1}\right\|$

$\leqslant \sup _{x \in \mathbb{E}_{1}(r)} \| \Gamma \underset{D f(z)}{\#}\left(h_{1}\left(z_{1}\right)^{\#}\right)-\Gamma_{D f(z)^{*}}^{\#-\left(h_{2}\left(z_{1}\right)^{\#}\right) \|}$

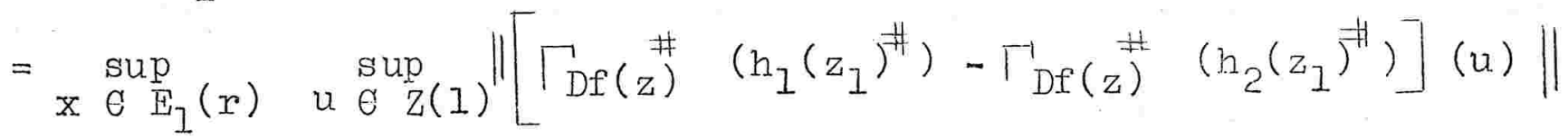

$=\sup _{x \in \mathbb{E}_{1}(r)} d\left(\Gamma_{D f(z)} \#\left(h_{1}\left(z_{1}\right)^{\#}\right), \Gamma_{D f(z)}\left(h_{2}\left(z_{1}\right)^{\#}\right)\right) \leqslant$ 


$$
\begin{aligned}
& \sup _{x \in E_{1}(r)} \lambda \cdot d\left(h_{1}\left(z_{1}\right)^{\#}, h_{2}\left(z_{1}\right)^{\#}\right)= \\
& =\lambda \sup _{x \in \mathbb{E}_{I}(r)} d\left(h_{I}\left(z_{I}\right)^{\#}, h_{2}\left(z_{1}\right)^{\#}\right) \\
& \text { Mas } x \in \mathbb{E}_{1}(r) \Rightarrow \bar{u}_{1} \in \mathbb{I}_{1}(r) \text {. Dar, } \\
& w_{g}\left(h_{1}\right) \cdots w_{g}\left(n_{2}\right) \leqslant \lambda_{z_{1}} \sup _{\mathbb{E}_{1}(r)}\left\|h_{1}\left(z_{1}\right)^{\#}-h_{2}\left(z_{1}\right)^{\#}\right\|= \\
& =\lambda \sup _{z_{1} \in F_{1}(r) \quad u \in z(1)}\left\|\left[h_{1}\left(z_{1}\right)-h_{2}\left(z_{1}\right)\right] \circ u\right\| \leqslant
\end{aligned}
$$

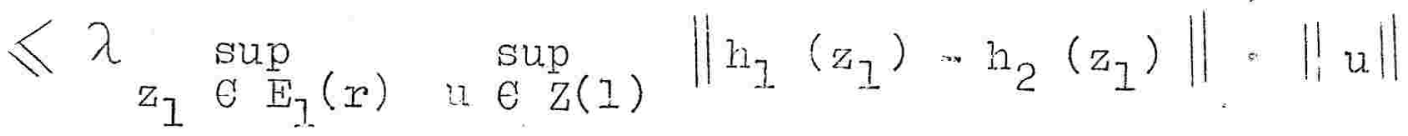

$$
\begin{aligned}
& =\quad \lambda \sup _{z_{1} \in \mathbb{E}_{1}(r)}\left\|h_{1}\left(z_{1}\right) \cdots h_{2}\left(z_{1}\right)\right\|=\lambda \cdot d\left(h_{1}, h_{2}\right)
\end{aligned}
$$

5.5 Uma generalização natural' da noção de ponto fixo hi perbólico para um difeomorfismo é o conceito de conjunto hiperbóli co. Seja, M uma variedade riemanniana de classe $C^{\infty}$ e dimensão $n$, $U \subset \mathbb{I}$ um aberto, $f: U \longrightarrow \mathbb{M}$ um mergulho (" embedding ") de classe $C^{k}, e \lambda$ um aubonjunto compacto invariante por $f$, j.sto $\epsilon$, tal que $f(\wedge)$.: $\wedge$ 。 Seja $\mathbb{T}_{\Lambda} \mathbb{M}$, a restrição do $\underline{f}$ brado tangente TMI a $\wedge$. O conjunto $\wedge$ é dito hiperbólico para $f$ se $\mathbb{T}_{\lambda} \mathbb{N}$ admite una decomposicão $\mathbb{T}_{\lambda} \mathbb{M}=\mathbb{E}^{\mathrm{U}} \oplus \mathbb{E}^{\mathrm{S}}$ (soma direta de Whitney) tal que:

(i) $\mathbb{E}^{\mathrm{U}} \circ \mathrm{E}^{\mathrm{S}}$ são invariantes por $\operatorname{Tf}(\mathrm{Tf}=$ diferencial de $\mathrm{f}$ )

(ii) existem constantes $c>0$ e $0<\zeta<1$ tais que, para todo $n \in \mathbb{N}$, 
$\max \left\{\left\|\mathrm{Tf}^{\mathrm{n}} / \mathrm{E}^{\mathrm{S}}\right\|,\left\|\mathrm{Tf}^{-n} / \mathrm{E}^{\mathrm{u}}\right\|\right\} \quad \mathrm{c} \boldsymbol{乙}^{\mathrm{n}}$

Em [6] , demonstra-se o Teorema da Variedade Estável para conjuntos hiperbólicos, que generaliza o teorema 4.14, e que garante a $\underline{e}$ xistência de uma familia $\left(W_{X}^{S}\right)_{X} \in \lambda$ de subvariedades de classe $\mathrm{C}^{\mathrm{k}}$, cada $\mathrm{W}_{\mathrm{X}}^{\mathrm{S}}$ tangente a $\mathrm{E}_{\mathrm{X}}^{\mathrm{S}} \mathrm{X}$ em $\mathrm{X}$, invariantes por $f$ (isto é, $\left.f\left(W_{X}^{S}\right) \subset W_{X}^{S}\right)$, e tais que existem $K>0$ e $0<\lambda<1$ para os quais : se $\mathrm{y}, \mathrm{z} \in \mathrm{W}_{\mathrm{X}}^{\mathrm{S}}$, então

$d\left(f^{n}(y), f^{n}(z)\right) \leqslant K \cdot \lambda^{n} \cdot d(y, z), \forall n \in \mathbb{N}$

5.6 Uma aplicação dos Teoremas das Variedades Estável e Instável para pontos fixos hiperbólicos de um difeomorfismo é a de monstração da existência das variedades estável e instável para uma órbita fechada hiperbólica de um campo de vetôres em una variedade II ( una órbita fechada é hiperbólica se a diferencial $\mathrm{Df}_{\mathrm{p}}$ de uma transformaça de Poincaré $f$ associada a $\hat{\emptyset}$ no ponto $p$ tem om $p$ um ponto fixo hiperbólico). Uma boa referência para o caso em que $\operatorname{dim} \mathbb{M}<\infty$ é [1]. 
B I B I I G $R A$ A I A

I R. Abraham e J. Marsden

Foundations of Mechanics

Benjamin, New York, 1967 :

2 R. Abraham e J, Robbin

Transversal Mappings and Flows

Benjamin, New York, 1967 .

3 E. A. Coddington e $\mathbb{N}$. Levinson

Theory of Ordinary Differential Equations

Mc Graw Hill - 1955

4 J. Dieudonné

Foundations of Modern Analysis Academic Press - 1960

5 P. Hartman

Ordinary Differential Equations

John Wiley and Sons, 1964

6 M. W. Hirsch e c. C. Pugh

Stable Manifolds and Hyperbolic Sets

Proceedings of the $A$. $\mathbb{M}, S$. Summer Institute on Global Analysis, vol. XIV -

- Berkeley, 1968 
7 R. B. Holmes

A Formula for the Spectral Radius of an Operator

The American Mathematical Monthly, vol. 75 (1968) - pag. 163

8

E. I. Lima

Análise Geométrica $7^{0}$ Colóquio Brasileiro de Matemática $-1969$

9 J. Palis Jr.

On the local structure of hyperbolic points in Banach Spaces. Anais da Academia Brasileira de Ciências, vol. $40(1968)-n \circ 3$

10

F. Riesz e B. Nagy

Leçons d'Analyse Fonctionelle Budapest - 1952

11 Robbin, J. W.

Stable Manifolds of Semi-Hyperbolic Fixed Points (a aparecer) 
Differentiable Dynamical Systems Bulletin of the A. M. S., vol. 73 (1967) , pag. 747

Seminários de Sistemas Dinâmicos 1970 - IMPA (a aparecer) 
$I N D I C E$

PAG.

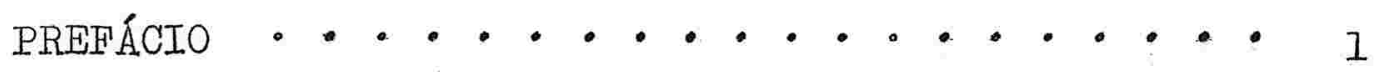

1. FUNÇÕES IIPSCHITZIANAS . . . . . . . 5

2. Funções UnI FormaMente cke . . . . . . 17

3. OPERADORES HIPERBÓII COS . . . . . . 32

4 . OS TEOREMAS DAS VARIEDADES ESTÁVEI E INSTÁVEI. 44

5. OBSERVAÇOES FINAIS . . . . . . . 92

BIBLIOGRAFIA . . . . . . . . . . . . 99 